\title{
Paired states of fermions in two dimensions with breaking of parity and time-reversal symmetries, and the fractional quantum Hall effect
}

\author{
N. Read and Dmitry Green \\ Departments of Physics and Applied Physics, Yale University, \\ P.O. Box 208120, New Haven, Connecticut 06520-8120
}

(February 1, 2008)

\begin{abstract}
We analyze pairing of fermions in two dimensions for fully-gapped cases with broken parity (P) and time-reversal $(\mathrm{T})$, especially cases in which the gap function is an orbital angular momentum ( $l$ ) eigenstate, in particular $l=-1$ (p-wave, spinless or spin-triplet) and $l=-2$ (d-wave, spinsinglet). For $l \neq 0$, these fall into two phases, weak and strong pairing, which may be distinguished topologically. In the cases with conserved spin, we derive explicitly the Hall conductivity for spin as the corresponding topological invariant. For the spinless p-wave case, the weak-pairing phase has a pair wavefunction that is asympototically the same as that in the Moore-Read (Pfaffian) quantum Hall state, and we argue that its other properties (edge states, quasihole and toroidal ground states) are also the same, indicating that nonabelian statistics is a generic property of such a paired phase. The strong-pairing phase is an abelian state, and the transition between the two phases involves a bulk Majorana fermion, the mass of which changes sign at the transition. For the d-wave case, we argue that the Haldane-Rezayi state is not the generic behavior of a phase but describes the asymptotics at the critical point between weak and strong pairing, and has gapless fermion excitations in the bulk. In this case the weak-pairing phase is an abelian phase which has been considered previously. In the p-wave case with an unbroken U(1) symmetry, which can be applied to the double layer quantum Hall problem, the weak-pairing phase has the properties of the 331 state, and with nonzero tunneling there is a transition to the Moore-Read phase. The effects of disorder on noninteracting quasiparticles are considered. The gapped phases survive, but there is an intermediate thermally-conducting phase in the spinless p-wave case, in which the quasiparticles are extended.
\end{abstract}

\section{INTRODUCTION}

Most theories of superconductivity, or more generally of superfluidity in fermion systems, depend on the concept of a paired ground state introduced by Bardeen, Cooper and Schrieffer (BCS) in 1957 [1, [2]. The ground state may be thought of loosely as a Bose condensate of pairs of particles, since such a pair can be viewed as a boson. Within BCS mean field theory, such a state forms whenever the interaction between the particles is attractive. For weak attractive interaction the elementary excitations are fermions (BCS quasiparticles), which can be created by adding or removing particles from the system, or in even numbers by breaking the pairs in the ground state, and the minimum excitation energy occurs at fermion wavevector near $k_{F}$, the Fermi surface that would exist in the normal Fermi liquid state at the same density of particles. There is also a collective mode, which is a gapless phonon-like mode in the absence of long-range interactions between the particles. This mode would also be present if one considered the pairs as elementary bosons, and would be the only elementary lowenergy excitation in that case. If the attractive interaction becomes strong, the energy to break a pair becomes large, and at all lower energies the system behaves like a Bose fluid of pairs. In the original BCS treatment, each pair of particles was in a relative s-wave $(l=0)$ state, and the minimum energy for a fermion excitation is then always nonzero. No phase transition occurs as the coupling strength is increased to reach the Bose fluid regime.

Not long after BCS, the theory was generalized to nonzero relative angular momentum $(l)$ pairing, and after a long search, p-wave pairing was observed in $\mathrm{He}^{3}$ [3]. It is believed that d-wave pairing occurs in heavy fermion and high $T_{c}$ superconductors. Some nonzero $l$ paired states generally have vanishing energy gap at some points on the Fermi surface (for weak coupling), while others do not. While the absence of a transition is wellknown in the s-wave case, it seems to be less well known that in some of these other cases, there is a phase transition as the coupling becomes more strongly attractive. One reason for this is that the strong-coupling regime must have a gap for all BCS quasiparticle excitations. But even when the weak coupling regime is fully gapped, there may be a transition, and these will be considered in this paper, in two dimensions.

In the paired states with non-zero $l$ there are many exotic pheneomena, especially in the p-wave case, due to the breaking of spin-rotation and spatial-rotation symmetries. These include textures in the order parameters for the pairing, such as domain walls, and quasiparticle excitations of vanishing excitation energy on these textures (zero modes) (these are reviewed in Ref. [3]). In transport, there may be Hall-type conductivities for conserved quantities, such as spin and energy, which are pos- 
sible because of the breaking of both parity $(\mathrm{P})$ and time reversal (T) symmetries. The breaking of these symmetries, and topological aspects of the paired state, are more crucial for the ocurrence of these effects than is the angular momentum of the pairing; the pairing need not be in a definite angular momentum state. Many of these effects have been discussed in remarkable papers by Volovik, of which a few are Refs. [4 8]. These are related to effects we will explore in this paper. We will make an effort to separate the effects related to breaking continuous symmetries spontaneously, which leads to familiar Goldstone mode physics, from those connected with topological effects, quasiparticle zero modes and Hall-type responses for unbroken symmetries.

In this paper we will make extensive use of the methods for BCS paired states, and consider the transitions between the weak and strong coupling regimes in two dimensions. In the weak-coupling regime, exotic phenomena are possible when parity and time reversal are broken. The results are applied to the fractional quantum Hall effect (FQHE) by using the composite fermion approach, to be reviewed below. We also consider effects of disorder on the phases and transitions, also within BCS mean field theory. In each Section, we try to make the initial discussion general and accessible for workers in many-body theory and superconductivity, before specializing to applications to the FQHE. In the remainder of this Introduction we will give an overview of the background and of the results of this paper.

We now review some background in the FQHE [9]. The original Laughlin states [10] occur at filling factors $\nu=1 / q$, with $q$ odd (the filling factor is defined as $\nu=n \Phi_{0} / B$, where $n$ is the density in two dimensions, $B$ is the magnitude of the magnetic field, and $\Phi_{0}=h c / e$ is the flux quantum). An early idea of Halperin [11] was to generalize the Laughlin states by assuming that under some conditions, the electrons could form pairs, which as charge-2 bosons could form a Laughlin state. A variety of such states were proposed 11 14. Since the Laughlin states for bosons occur at filling factors for the lowest Landau level (LLL) $\nu_{b}=1 / m$, with $m$ even, and the filling factor for the electrons is related to that for the bosons by $\nu=4 \nu_{b}$, the electron filling factor is either of the form $\nu=1 / q$ ( $q$ an integer $)$, or $\nu=2 / q, q$ odd. In particular, these values include $\nu=1 / 2,1 / 4, \ldots$, which do not correspond to incompressible states in the usual hierarchy scheme [15], because the filling factors in the latter always have odd denominators (when common factors have been removed).

The relation of the paired states in the FQHE to those in superfluidity theory becomes much closer once one introduces the notion of composite particles 16 30]. A simple, direct formal approach is to use a flux attachment or Chern-Simons transformation (see e.g. Ref. [25] in particular), which represents each particle as (in the case of most interest) a fermion plus an integer number $\tilde{\phi}$ of $\delta$-function flux tubes. After the transformation, the system can be represented by an action that includes a Chern-Simons term for a U(1) gauge field, that couples to the Fermi field. We refer to this as the CS fermion approach. The net magnetic field seen by the fermions is the sum of the external field and the $\delta$-function fluxes on the other particles. In a mean field treatment, given a uniform density of particles, this produces a net average field that vanishes when the filling factor is $\nu=1 / \tilde{\phi}$. In this case the fermions can form a Fermi sea [25], or they could form a BCS paired state. Some of the existing paired FQHE trial wavefunctions can be interpreted this way, as pointed out in Ref. [14], and others can be constructed by analogy 14.

A more physical way of looking at the formation of the composite particles, particularly when they are considered as the elementary excitations of the system, is as bound states of one of the underlying particles (or particles for short), and $\tilde{\phi}$ vortices in the particle wavefunction 21,26]. The bound states, which correspond to the CS fermions, again behave as fermions in zero net field if the particles obey Fermi statistics and $\tilde{\phi}$ is even, (or if the particles obey Bose statistics and $\tilde{\phi}$ is odd) and $\nu=1 / \tilde{\phi}$. Note that we will consistently use the term "particle" for the underlying particles, and "fermion" for the CS or composite fermions (bound states). Some statements apply also when the transformed particles are bosons (obtained by interchanging the words "even" and "odd" in the preceding definitions), in which case we refer to composite particles. It is generally more important to keep track of the statistics and net magnetic field seen by the composite particles than those of the underlying particles. Recent work has formalized the bound state picture, and improved our understanding [27 30]. However, the results of the CS approach remain valid, and because that approach is simple to use, and we will mainly require only a mean field picture here, we assume that that is the approach we are implicitly using.

The Laughlin states can be viewed as Bose condensates of composite bosons in zero net magnetic field 18,20,21. Because the bosons are coupled to a gauge field (in the CS approach, the CS gauge field), vortex excitations cost only a finite energy, but there is still an effective Meissner effect for the CS gauge field. Because the flux of the CS gauge field is related to the particle density, a vortex carries a fractional charge and corresponds to Laughlin's fractionally-charged quasiparticles [10] (we refer to such excitations as FQHE quasiparticles). Hence the Meissner effect in the superfluid becomes the incompressiblity of the FQHE state (there is of course no Meissner effect or superfluidity in the response to the electromagnetic field). Similarly, when pairing of composite fermions occurs in zero net magnetic field, the state becomes incompressible [14]. In contrast, the Fermi liquid state of Halperin, Lee and one of the authors [25] has no Meissner effect for the CS field and is compressible 25]. 
The wavefunction proposed by Haldane and Rezayi (HR) in Ref. 13 is a spin-singlet paired state, which can be interpreted as a d-wave paired state of composite fermions [14]. Moore and Read (MR) [14 proposed a p-wave paired state (the "Pfaffian state") of spinless electrons with a structure analogous to the HR state. Both states can occur for filling factors $\nu=1 / 2,1 / 4, \ldots$ The HR state was proposed as an explanation for the observed $\nu=5 / 2 \mathrm{QH}$ state [31], which collapses when a parallel component of the magnetic field is applied, suggesting that it is a spin singlet. However, it was also proposed later that the $5 / 2$ state is the $\mathrm{MR}$ state [32]. In both proposals, the LLL is filled with electrons of both spins, and the paired FQHE state describes only the electrons in the first excited LL. The latter proposal is supported by recent numerical work 33,34. The collapse of the state under a parallel magnetic field must then be due to another mechanism, involving the effects of the finite thickness of the single-particle wavefunctions in the direction perpendicular to the two-dimensional layer, which is poorly understood at present. Another paired state with a similar interpretation is the 331 state 11$]$, which can be viewed as a p-wave paired state of two-component composite fermions $13,35,36$. It is likely that this is closely related to a FQHE state observed in double-layer and single-thick-layer systems at $\nu=1 / 2$ 37, 38.

Moore and Read 14 suggested that nonabelian statistics might occur in $\mathrm{QH}$ states, and the Pfaffian state was proposed as an example. Nonabelian statistics means that the space of states for a collection of quasiparticles at fixed positions and quantum numbers is degenerate, and when quasiparticles are exchanged adiabatically (for which we need the system to have an energy gap for all excitations), the effect is a matrix operation on this space of degenerate states. This generalizes the idea of fractional statistics, in which the effect of an exchange is a phase factor and the states as specified are nondegenerate; when the phase for an elementary exchange is \pm 1 , one has bosons or fermions. The arguments in MR that this would occur were based heavily on the identification of the many-particle wavefunctions in the FQHE as chiral correlators (conformal blocks) in two-dimensional conformal field theory, which possess similar properties under monodromy (analytic continuation in their arguments). It was expected that an effective field theory description of these effects would be based on nonabelian ChernSimons theories, which are known to be connected with conformal field theory, and to lead to nonabelian statistics [39]. In the MR state, and other paired states, there are, apart from the usual Laughlin quasiparticles which contain a single flux quantum, also finite energy vortex excitations containing a half flux quantum [14, and it is these which, in the MR state, are supposed to possess nonabelian statistics properties.

Evidence for nonabelian statistics in the MR (Pfaffian) state accumulated in later work 40 44, which in- vestigated the spectrum of edge states, quasihole states, and ground states on the torus (periodic boundary conditions), all of which were obtained as the zero-energy states for the three-body Hamiltonian of Greiter et al., for which the MR state is the exact unique ground state [32. The states found agreed precisely with the expectations based on conformal field theory. There was also evidence for similar effects in the HR state [41,42, 44, however the interpretation was problematic because the natural conformal field theory for the bulk wavefunctions is nonunitary and therefore cannot directly describe the edge excitations, as it does in other cases such as the MR state. Some solutions to this were proposed [42,45]. Explicit derivations of nonabelian statistics and of effective theories have been obtained later for the MR state 46, 47. The 331 state [11] is an abelian state, which can be viewed as a generalized hierarchy state 48,49, as is evident from the plasma form of the state, and these two descriptions are related by a bosonization mapping 42,44]. The hierarchy states and their generalizations possess abelian statistics properties, which can be characterized by a (Bravais) lattice 48,49]. Thus incompressible FQHE states in general can be divided into two classes, termed abelian and nonabelian. It is clear that in Halperin's picture 11] of bound electron pairs which form a Laughlin state of charge 2 bosons, the properties will be abelian and are simply described by a one-dimensional lattice, in the language of Ref. [48] (they are the simplest examples of a class of abelian states in which the objects that Bose condense contain more than one electron plus some vortices, while the hierarchy states, and all generalizations considered in Ref. [48], have condensates involving single electrons).

In spite of the work that has been done, one may still ask questions such as what is the microscopic mechanism, in terms of composite fermions, for the degeneracies of FQHE quasiparticle states that is the basis for nonabelian statistics, and whether it is robust against changes in the Hamiltonian. A similar question is about the effects of disorder. These will be addressed in this paper, by a direct and simple analysis of the paired states using BCS mean field theory, and developments such as the Bogoliubov-de Gennes equations [50]. We find that the nontrivial paired FQHE states are related to the weak-coupling regime (or more accurately, the weakpairing phase); in particular the MR and 331 states have wavefunctions that contain the generic long-distance behavior in spinless and spin-triplet p-wave weak-pairing phases, respectively. In contrast, the strong-coupling regimes, or strong-pairing phases, lead in the FQHE to the Halperin type behavior. There is a similar picture in the spin-singlet d-wave case, except that the HR state, which might have been expected to represent the weak-pairing phase, is in fact at the phase transition, and therefore is gapless in the bulk. The weakpairing phases are topologically nontrivial, and possess 
edge states and nontrivial quantum numbers on the vortices (FQHE quasiparticles); the spinless p-wave case (MR phase) is nonabelian, while the spinful p-wave case (with unbroken U(1) symmetry), and the spin-singlet dwave case are abelian states, which we characterise. We also consider the effect of tunneling on the double layer system which is the best candidate for realising the 331 state, and show that the phase diagram includes a MR phase. The theory also leads to a description of the critical theories at the transitions, at least within a mean field picture. The role of fluctuations, and the full effective field theories at these transitions, remain to be understood.

We also consider disorder within the same approximation, making use of recent results on noninteracting BCS quasiparticles with disorder [51 58], and in particular we find that in the spinless p-wave case, there can be an intermediate phase with the thermal properties of a metal, between the two localized phases which correspond to the weak (MR) and strong-pairing phases of the pure case. A disordered version of the MR phase still exists in the presence of disorder, though its properties, including nonabelian statistics, may become more subtle.

One further issue which we discuss is the transport coefficients of Hall type for various conserved quantities, especially spin and energy. Concentrating on the quantities that are related to unbroken symmetries, we derive explicitly the values of these conductivities in the spin case, for spin-singlet and triplet states, and show that they are quantized in the sense of being given by topological invariants (in the disordered cases, we do not prove this directly). There have been claims that, in some sense or other, the ordinary Hall conductivity for charge (particle number) transport takes a nonzero quantized value in a

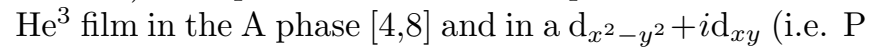
and $\mathrm{T}$ violating d-wave) superconductor [59,60]. It seems unlikely to us that these claims are correct, if the Hall conductivity is defined in the usual way, as the current response to an external (or better, to the total) electric field, taking the wavevector to zero before the frequency. While one can set up a detailed calculation, using a conserving approximation as in the Appendix, which duly includes the collective mode effect in this case where the symmetry corresponding to the transported quantity is broken, we prefer to give here a more direct and appealing argument. This works in the case where pairing is assumed to occur in a system of interacting fermions of mass $m$ with Galilean invariance in zero magnetic field, as in most models of pairing. If one considers the linear response to an imposed uniform but finite frequency $(\omega)$ electric field, then there are well-known arguments that the conductivity is simply $\sigma_{\mu \nu}(\omega)=n e^{2} \delta_{\mu \nu} /(m \omega+i \eta)(\mu$, $\nu=x, y$, and $\eta$ is a positive infinitesimal). This arises from the so-called diamagnetic term, and the contribution of the two-point current-current function vanishes in this limit. This is independent of interactions, and hence also of whether the interactions produce pairing or not. The result can be understood as the contribution of the center of mass, which is accelerated by the applied uniform electric field, while the relative motion of the particles is unaffected, as a consequence of Galilean invariance. There is clearly no Hall conductivity. (However, the similar calculation in a magnetic field produces the standard Hall conductivity, and is an aspect of Kohn's theorem.) We may be curious about non-Galilean invariant models, and whether a paired state could have a quantized Hall conductivity as claimed. But if it were quantized, it would be invariant under any continuous change in the Hamiltonian that preserves the gap. Hence, in the ground state of any model that can be continuously connected to the Galilean-invariant models, the Hall conductivity must either, if quantized, vanish, or else vary continuously and not be quantized.

The plan of the remainder of this paper is as follows. In Section [I], we first consider the ground state in a system of spinless fermions with p-wave pairing, for the infinite plane and for periodic boundary conditions (a torus). We show that a transition occurs between weak and strongpairing phases, which can be distinguished topologically in momentum space, or by the number of ground states on the torus for even and odd particle number. In Subsec. II B, we consider the system in the presence of edges and vortices. We argue that there are chiral fermions on an edge, and degeneracies due to zero modes on vortices, when these occur in the weak-pairing phase. In Subsec. II , we show how the results for ground states can be extended to other geometries, such as the sphere. Section II as a whole shows that the properties of the weak-pairing phase, the ground state degeneracies, chiral edge states and degeneracies of vortices agree with those expected in the MR phase in the FQHE. The strong-pairing phase has the properties expected in the Halperin paired states. In Sec. III, we consider the case of spin-triplet pairing, with applications to the double-layer FQHE system. There is a weak-pairing phase with the properties of the 331 state, and also a distinct phase with the properties of the MR state. In Sec. IV, we consider spin-singlet d-wave pairing. In Subsec. IV A, we argue that the HR state corresponds to the transition point between weak- and strong-pairing, and so has gapless fermions in the bulk. Then we analyse the generic weak-pairing $\mathrm{d}$-wave phase, and argue that it corresponds to an abelian FQHE state, with a spin-1/2 doublet of chiral Dirac fermions on the edge, which has also been constructed previously. We also discuss here (in Subsec. IV ) general arguments for the existence of the edge states and other effects, based on Hall-type conductivities and induced CS actions in the bulk, for all the paired states. In Sec. V, we discuss the effects of disorder on all the transitions and phases. The explicit calculations of the Hall spin conductivity, in the pure systems, are given in the Appendix.

A brief announcement of our results for pure systems 
was made in Ref. 61. Some of the results have also been found independently by others 62 .

\section{COMPLEX P-WAVE PAIRING OF SPINLESS OR SPIN-POLARIZED FERMIONS}

In this Section, we first set up the BCS effective quasiparticle Hamiltonian, and review its solution by Bogoliubov transformation. We show that this leads to the existence of a transition between distinct phases, which we label weak- and strong-pairing. They are distinguished topologically. The weak-pairing phase is tentatively identified with the MR phase because of its behavior in position space. This is extended to the torus (periodic boundary conditions), where we find three ground states for $N$ even, one for $N$ odd, in the weak-pairing phase, in agreement with the MR state. In Subsec. II B, we show that the BCS quasiparticles at long wavelengths near the transition are relativistic Majorana fermions, and use this to analyze the Bogoliubov-de Gennes equations for domain walls (edges) and vortices, again arguing that the results agree with those obtained for the MR state. In Subsec. II , which may be omitted on a first reading, we show how p-wave pairing on a general curved surface can be handled mathematically, and that the ground states agree with conformal blocks, as expected from MR.

\section{A. Weak and strong pairing phases}

First we recall the relevant parts of BCS mean field theory [2]. The effective Hamiltonian for the quasiparticles is

$$
K_{\mathrm{eff}}=\sum_{\mathbf{k}}\left[\xi_{\mathbf{k}} c_{\mathbf{k}}^{\dagger} c_{\mathbf{k}}+\frac{1}{2}\left(\Delta_{\mathbf{k}}^{*} c_{-\mathbf{k}} c_{\mathbf{k}}+\Delta_{\mathbf{k}} c_{\mathbf{k}}^{\dagger} c_{-\mathbf{k}}^{\dagger}\right)\right]
$$

where $\xi_{\mathbf{k}}=\varepsilon_{\mathbf{k}}-\mu$ and $\varepsilon_{\mathbf{k}}$ is the single-particle kinetic energy and $\Delta_{\mathbf{k}}$ is the gap function. For the usual fermion problems, $\mu$ is the chemical potential, but may not have this meaning in the FQHE applications. At small $\mathbf{k}$, we assume $\varepsilon_{\mathbf{k}} \simeq k^{2} / 2 m^{*}$ where $m^{*}$ is an effective mass, and so $-\mu$ is simply the small $\mathbf{k}$ limit of $\xi_{\mathbf{k}}$. For complex p-wave pairing, we take $\Delta_{\mathbf{k}}$ to be an eigenfunction of rotations in $\mathbf{k}$ of eigenvalue (two-dimensional angular momentum) $l=-1$, and thus at small $\mathbf{k}$ it generically takes the form

$$
\Delta_{\mathrm{k}} \simeq \hat{\Delta}\left(k_{x}-i k_{y}\right),
$$

where $\hat{\Delta}$ is a constant. For large $\mathbf{k}, \Delta_{\mathbf{k}}$ will go to zero. The $c_{\mathbf{k}}$ obey $\left\{c_{\mathbf{k}}, c_{\mathbf{k}^{\prime}}^{\dagger}\right\}=\delta_{\mathbf{k k}^{\prime}}$; we work in a square box of side $L$, and consider the role of the boundary conditions and more general geometries later.

The normalized ground state of $K_{\text {eff }}$ has the form

$$
|\Omega\rangle=\prod_{\mathbf{k}}^{\prime}\left(u_{\mathbf{k}}+v_{\mathbf{k}} c_{\mathbf{k}}^{\dagger} c_{-\mathbf{k}}^{\dagger}\right)|0\rangle,
$$

where $|0\rangle$ is the vacuum containing no fermions. The prime on the product indicates that each distinct pair $\mathbf{k},-\mathbf{k}$ is to be taken once. (We will later consider the precise behavior at $\mathbf{k}=\mathbf{0}$.) The functions $u_{\mathbf{k}}$ and $v_{\mathbf{k}}$ are complex and obey $\left|u_{\mathbf{k}}\right|^{2}+\left|v_{\mathbf{k}}\right|^{2}=1$ to ensure $\langle\Omega \mid \Omega\rangle=$ 1. They are determined by considering the Bogoliubov transformation

$$
\begin{aligned}
& \alpha_{\mathbf{k}}=u_{\mathbf{k}} c_{\mathbf{k}}-v_{\mathbf{k}} c_{-\mathbf{k}}^{\dagger}, \\
& \alpha_{\mathbf{k}}^{\dagger}=u_{\mathbf{k}}^{*} c_{\mathbf{k}}^{\dagger}-v_{\mathbf{k}}^{*} c_{-\mathbf{k}},
\end{aligned}
$$

so that $\left\{\alpha_{\mathbf{k}}, \alpha_{\mathbf{k}^{\prime}}^{\dagger}\right\}=\delta_{\mathbf{k k}^{\prime}}$ and $\alpha_{\mathbf{k}}|\Omega\rangle=0$ for all $\mathbf{k}$. By insisting that $\left[\alpha_{\mathbf{k}}, K_{\text {eff }}\right]=E_{\mathbf{k}} \alpha_{\mathbf{k}}$ for all $\mathbf{k}$, which implies that

$$
K_{\mathrm{eff}}=\sum_{\mathbf{k}} E_{\mathbf{k}} \alpha_{\mathbf{k}}^{\dagger} \alpha_{\mathbf{k}}+\text { const }
$$

with $E_{\mathbf{k}} \geq 0$, one obtains (the simplest form of) the Bogoliubov-de Gennes (BdG) equations,

$$
\begin{aligned}
& E_{\mathbf{k}} u_{\mathbf{k}}=\xi_{\mathbf{k}} u_{\mathbf{k}}-\Delta_{\mathbf{k}}^{*} v_{\mathbf{k}} \\
& E_{\mathbf{k}} v_{\mathbf{k}}=-\xi_{\mathbf{k}} v_{\mathbf{k}}-\Delta_{\mathbf{k}} u_{\mathbf{k}} .
\end{aligned}
$$

These imply that

$$
\begin{aligned}
E_{\mathbf{k}} & =\sqrt{\xi_{\mathbf{k}}^{2}+\left|\Delta_{\mathbf{k}}\right|^{2}}, \\
v_{\mathbf{k}} / u_{\mathbf{k}} & =-\left(E_{\mathbf{k}}-\xi_{\mathbf{k}}\right) / \Delta_{\mathbf{k}}^{*}, \\
\left|u_{\mathbf{k}}\right|^{2} & =\frac{1}{2}\left(1+\frac{\xi_{\mathbf{k}}}{E_{\mathbf{k}}}\right), \\
\left|v_{\mathbf{k}}\right|^{2} & =\frac{1}{2}\left(1-\frac{\xi_{\mathbf{k}}}{E_{\mathbf{k}}}\right) .
\end{aligned}
$$

The functions $u_{\mathbf{k}}$ and $v_{\mathbf{k}}$ are determined only up to an overall phase for each $\mathbf{k}$, so they can be multiplied by a k-dependent phase, $u_{\mathbf{k}} \rightarrow e^{i \phi_{\mathbf{k}}} u_{\mathbf{k}}, v_{\mathbf{k}} \rightarrow e^{i \phi_{\mathbf{k}}} v_{\mathbf{k}}$ without changing any physics. One may adopt a convention that one of $u_{\mathbf{k}}$ and $v_{\mathbf{k}}$ is real and positive; in either case the other must be odd and of p-wave symmetry under rotations. We do not use such a convention explicitly because there is no single choice that is convenient for all that follows.

Because of Fermi statistics, which imply $\left(c_{\mathbf{k}}^{\dagger}\right)^{2}=0$, we can rewrite the ground state (up to a phase factor) as

$$
|\Omega\rangle=\prod_{\mathbf{k}}\left|u_{\mathbf{k}}\right|^{1 / 2} \exp \left(\frac{1}{2} \sum_{\mathbf{k}} g_{\mathbf{k}} c_{\mathbf{k}}^{\dagger} c_{-\mathbf{k}}^{\dagger}\right)|0\rangle,
$$

where $g_{\mathbf{k}}=v_{\mathbf{k}} / u_{\mathbf{k}}$. Then the wavefunction for the component of the state with $N$ fermions ( $N$ even) is, up to an $N$-independent factor, 


$$
\begin{aligned}
\Psi\left(\mathbf{r}_{1}, \ldots, \mathbf{r}_{N}\right)= & \frac{1}{2^{N / 2}(N / 2) !} \sum_{P} \operatorname{sgn} P \\
& \times \prod_{i=1}^{N / 2} g\left(\mathbf{r}_{P(2 i-1)}-\mathbf{r}_{P(2 i)}\right),
\end{aligned}
$$

where $g(\mathbf{r})$ is the inverse Fourier transform of $g_{\mathbf{k}}$,

$$
g(\mathbf{r})=L^{-2} \sum_{\mathbf{k}} e^{i \mathbf{k} \cdot \mathbf{r}} g_{\mathbf{k}}
$$

and $P$ runs over all permutations of $N$ objects. (For fermions with spin, this appears on p. 48 in Ref. [2].) The right-hand side of Eq. (12) is a Pfaffian, which for a general $N \times N$ matrix with elements $M_{i j}(N$ even) is defined by

$$
\operatorname{Pf} M=\frac{1}{2^{N / 2}(N / 2) !} \sum_{P} \operatorname{sgn} P \prod_{i=1}^{N / 2} M_{P(2 i-1) P(2 i)},
$$

or as the square root of the determinant, $\operatorname{Pf} M=$ $\sqrt{\operatorname{det} M}$, for $M$ antisymmetric.

We now consider the form of the solutions to the above equations. In the usual BCS problem, the functions $\Delta_{\mathbf{k}}$ and $\varepsilon_{\mathbf{k}}$ are found self-consistently from the gap equation (including Hartree-Fock effects), and $\mu$ would be determined by specifying the fermion density. However, we are not interested in all these details, but in the nature of the possible phases and in the transitions between them. We expect that the phases can be accessed by changing the interactions and other parameters of the problem, but we will not address this in detail. In particular, some phases may require that the interactions be strong, while BCS theory is usually thought of as weak coupling. We will nonetheless continue to use the BCS mean field equations presented above, as these give the simplest possible view of the nature of the phases.

From Eqs. (9,10), we see that since $E_{\mathbf{k}}-\left|\xi_{\mathbf{k}}\right| \rightarrow 0$ as $\mathbf{k} \rightarrow \mathbf{0}$, we will have one of three possibilities for the behavior at small $\mathbf{k}$, which will turn out to govern the phases. As $\mathbf{k} \rightarrow 0$, either (i) $\xi_{\mathbf{k}}>0$, in which case $\left|u_{\mathbf{k}}\right| \rightarrow 1,\left|v_{\mathbf{k}}\right| \rightarrow 0$, or (ii) $\xi_{\mathbf{k}}<0$, in which case $\left|u_{\mathbf{k}}\right| \rightarrow$ $0,\left|v_{\mathbf{k}}\right| \rightarrow 1$, or (iii) $\xi_{\mathbf{k}} \rightarrow 0$, in which case $\left|u_{\mathbf{k}}\right|$ and $\left|v_{\mathbf{k}}\right|$ are both nonzero. We will term the first case the strong-pairing phase, the second case weak-pairing, while the third case, in which the dispersion relation of the quasiparticles is gapless, $E_{\mathbf{k}} \rightarrow 0$ as $\mathbf{k} \rightarrow 0$, is the phase transition between the weak and strong pairing phases. Thus for $\mu$ positive, the system is in the weak pairing phase, for $\mu$ negative, the strong-pairing phase, and the transition is at $\mu=0$ within our parametrization. The reason for these names will be discussed below.

We now discuss the two phases and the transition in more detail. We expect that the large $\mathbf{k}$ behavior of $\xi_{\mathbf{k}}$ and $\Delta_{\mathbf{k}}$ that would be produced by solving the full system of equations will not be affected by the occurrence of the transition which involves the small k's only. Note that, at large $\mathbf{k}, v_{\mathbf{k}} \rightarrow 0$ and $\left|u_{\mathbf{k}}\right| \rightarrow 1$, which ensures in particular that the fermion number, which is governed by $\bar{N}=\sum_{\mathbf{k}} \bar{n}_{\mathbf{k}}$, with

$$
\left\langle c_{\mathbf{k}}^{\dagger} c_{\mathbf{k}}\right\rangle=\bar{n}_{\mathbf{k}}=\left|v_{\mathbf{k}}\right|^{2},
$$

will converge. Also we assume that $E_{\mathbf{k}}$ does not vanish at any other $\mathbf{k}$, which is generically the case in the $l=$ -1 states. Thus within our mean field theory we can ignore the dependence of the functions $\xi_{\mathbf{k}}$ and $\Delta_{\mathbf{k}}$ on the distance from the transition, which we can represent by $\mu$.

In the strong-pairing phase, $\mu<0$, we have $v_{\mathbf{k}} \propto$ $k_{x}-i k_{y}$, as $\mathbf{k} \rightarrow 0$. Then the leading behavior in $g_{\mathbf{k}}=v_{\mathbf{k}} / u_{\mathbf{k}}$ is $\propto k_{x}-i k_{y}$, which is real-analytic in $k_{x}$ and $k_{y}$. If $g_{\mathbf{k}}$ is real-analytic in a neighborhood of $\mathbf{k}=0$, then $g(\mathbf{r})$ will fall exponentially for large $\mathbf{r}, g(\mathbf{r}) \sim e^{-r / r_{0}}$, but even if not it will fall rapidly compared with the other cases below. Thus we term this phase the strong-pairing phase because the pairs in position space are tightly bound in this sense. Note that this region is $\mu<0$, which would only be reached for strongly attractive coupling of the fermions (we disregard the possibility of other nonpairing phases for such couplings). Because $\bar{n}_{\mathbf{k}}=\left|v_{\mathbf{k}}\right|^{2}$, there is little occupation of the small $\mathbf{k}$ values in this phase.

In the weak-pairing phase, $u_{\mathbf{k}} \propto k_{x}+i k_{y}$ for $\mathbf{k} \rightarrow 0$, and so $g_{\mathbf{k}} \propto 1 /\left(k_{x}+i k_{y}\right)$, which gives

$$
g(\mathbf{r}) \propto 1 / z
$$

for large $\mathbf{r}$, where $z=x+i y$. This long tail in $g(\mathbf{r})$ is the reason for the term "weak pairing". This is exactly the behavior of $g(\mathbf{r})$ in the Moore-Read (MR) Pfaffian state in the FQHE. In the latter this form, $1 / z$, is valid for all distances. We will therefore try to argue that all the universal behavior associated with the MR state is generic in the weak-pairing phase, when the theory is applied to the paired FQHE states. Notice that in the weak-pairing phase, the occupation numbers of the small $\mathbf{k}$ states approaches 1 . Of course, this is also the behavior of the Fermi sea. When the attractive coupling is weak, one would expect the weak pairing phase. If we imagine that only the magnitude of the coupling is varied, then when it is small and negative, the BCS weak-coupling description is valid, and $E_{\mathbf{k}}$ has a minimum at $k_{F}$. This is not the weak-strong transition. Close to the latter transition, $E_{\mathbf{k}}$ has a minimum at $\mathbf{k}=\mathbf{0}$. As the coupling weakens, a point is reached at which the minimum moves away from $\mathbf{k}=\mathbf{0}$, and eventually reaches $|\mathbf{k}|=k_{F}$ when the coupling strength is zero and the transition to the Fermi sea (or Fermi liquid phase) takes place. Thus the weak-pairing phase does not require that the coupling be weak, but is continuously connected to the weak-coupling $\mathrm{BCS}$ region. 
At the weak-strong transition, $\mu=0$, we find at small $\mathbf{k}$ that $E_{\mathbf{k}}=\left|\Delta_{\mathbf{k}}\right|,\left|u_{\mathbf{k}}\right|^{2},\left|v_{\mathbf{k}}\right|^{2} \rightarrow 1 / 2$, and $g_{\mathbf{k}}=\left(k_{x}-\right.$ $\left.i k_{y}\right) /|\mathbf{k}|$. The singular behavior of $g_{\mathbf{k}}$ leads to

$$
g(\mathbf{r}) \propto \frac{1}{z|z|}
$$

for large $\mathbf{r}$. This behavior is intermediate between those of the two phases. Because, like the strong-pairing behavior, it is not a rational function of $z$, it does not correspond to a "nice" LLL FQHE wavefunction. Also as $\mu \rightarrow 0$, the length scale on which the asympototics of the two phases is valid ( $r_{0}$ on the strong pairing side) diverges. This length should not necessarily be identified with the coherence length $\xi_{0}$ of the paired state. If the latter is viewed as "the size of a pair", it may be better associated with the decay of the inverse Fourier transform of $\left\langle c_{\mathbf{k}} c_{-\mathbf{k}}\right\rangle=u_{\mathbf{k}}^{*} v_{\mathbf{k}}=-\Delta_{\mathbf{k}} / E_{\mathbf{k}}$, which develops long-range behavior only at the transition. Also, for some purposes the relevant function may be $\Delta_{\mathbf{k}}$, which is always nonsingular at $\mathbf{k}=0$, and does not vanish at the transition. For the purpose of making contact with the existing FQHE wavefunctions, $g(\mathbf{r})$ is the appropriate quantity.

The distinction between the two phases, which we can view as requiring the existence of the phase transition, does not lie in symmetries, unlike many phase transitions; instead, it is topological in nature [4. Within the mean field treatment, this can be seen in terms of the topology of the functions $u_{\mathbf{k}}, v_{\mathbf{k}}$, or of $E_{\mathbf{k}}, \Delta_{\mathbf{k}}$. We recall that $u_{\mathbf{k}}$, $v_{\mathbf{k}}$ obey $\left|u_{\mathbf{k}}\right|^{2}+\left|v_{\mathbf{k}}\right|^{2}=1$, and that multiplying them both by the same phase is irrelevant. These conditions imply that they can be viewed as spinor (or "homogeneous") coordinates for a 2-sphere $S^{2}$, for which two real coordinates are sufficient for a non-redundant parametrization of any neighborhood. As usual, we view $u=1, v=0$ as the north pole $\mathcal{N}, u=0, v=1$ as the south pole $\mathcal{S}$. We can alternatively parametrize the sphere using the unit vector $\mathbf{n}_{\mathbf{k}}=\left(\operatorname{Re} \Delta_{\mathbf{k}},-\operatorname{Im} \Delta_{\mathbf{k}}, \xi_{\mathbf{k}}\right) / E_{\mathbf{k}}$, and this agrees with the parametrization by $u_{\mathbf{k}}, v_{\mathbf{k}}$. This is essentially the "pseudospin" point of view of Anderson [63], who described the s-wave BCS state as a spherical domain wall in the pseudospin in $\mathbf{k}$-space, of radius $k_{F}$. Because $v_{\mathbf{k}} \rightarrow 0$ as $\mathbf{k} \rightarrow \infty$ in any direction, we can add a point at infinity in $\mathbf{k}$ space and view it also as topologically a 2 -sphere, with the point at infinity at $\mathcal{N}$. The functions $u_{\mathbf{k}}, v_{\mathbf{k}}$ thus describe a mapping from $S^{2}(\mathbf{k}-$ space) to $S^{2}$ (spinor space), with $\mathcal{N}$ always mapping to $\mathcal{N}$. Such maps are classified topologically into equivalence classes, called homotopy classes, such that maps in the same class can be continuously deformed into each other. The special cases of maps from $S^{n}, n=1,2, \ldots$, to any space $X$ define the homotopy groups $\pi_{n}(X)$ (using a general method of producing a group structure on the equivalence classes with base points, which here are $\mathcal{N})$. In our case, $\pi_{2}\left(S^{2}\right)=\mathbf{Z}$, the group of integers. By inspection, we find that in the strong-pairing phase, the map is topologically trivial. In this phase the map can be deformed to the topologically-trivial map that takes all $\mathbf{k}$ to $\mathcal{N}$. In any topologically nontrivial map, as $\mathbf{k}$ varies over the plane plus point at infinity, $u, v$ (or $\mathbf{n}$ ) range over the whole sphere. Indeed, the number of times that a given point $\neq \mathcal{N}$, such as $\mathcal{S}$, is taken by $u, v$ must be at least $|m|$, for a map in the class labelled by the integer $m$. In our case, in the weak-pairing phase the map passes through $\mathcal{S}$ at least once, namely when $\mathbf{k}=0$, and possibly (most likely) only once, and we can choose to identify the class with $m=1$. This nontrivial topology in $\mathbf{k}$-space associated with non-s-wave weak-pairing ground states in two dimensions was pointed out by Volovik 化. We also note that the $\mathrm{p}$-wave weak-pairing phase map is a topologically non-trivial texture of the pseudospin that is also familiar in physics as a two-dimensional instanton, or a skyrmion in a $2+1$ dimensional system, in position space, in the $\mathrm{O}(3)$ nonlinear sigma model. Since it is impossible to pass smoothly between $m=0$ and $m=1$, the map $u_{\mathbf{k}}, v_{\mathbf{k}}$ must be discontinuous at the transition, which is what we found. In fact, $|u|^{2}$ and $|v|^{2}$ tend to $1 / 2$ as $\mathbf{k} \rightarrow 0$ at the transition, which corresponds to points on the equator. The simplest form of such a map is one that covers just the northern hemisphere, centered on $\mathcal{N}$.

The topological distinction between weak- and strongpairing phases is typical for the fully-gapped complex non-s-wave paired states, and not only when the gap function is an angular momentum eigenstate. For contrast, note that any s-wave state yields a topologicallytrivial $(m=0)$ map. For the s-wave case, it is well known that $u_{\mathbf{k}}$ and $v_{\mathbf{k}}$ can be chosen real, and continuously interpolate between the weak and strong coupling limits without a phase transition. Also, in three dimensions, the relevant homotopy group for the present spinless case is $\pi_{3}\left(S^{2}\right)=\mathbf{Z}$, and so nontrivial states do exist in principle, the simplest of which is the Hopf texture in $\mathbf{k}$-space. The usual s-wave state is again topologically trivial. Note that in the usual BCS model with a separable interaction, the gap function vanishes outside a thin shell around the Fermi surface, which makes the maps slightly discontinuous. We consider only interactions that are continuous in $\mathbf{k}$-space, for which the gap function and the map are continuous except at the transition.

To close this subsection, we consider (as promised earlier) the effect on the ground states of the boundary conditions and the total fermion number. That is, we use a two-dimensional (Bravais) lattice, and consider a system on a plane with points differing by a lattice vector identified, generally described as periodic or generalized periodic boundary conditions, or by saying that the system is a torus.

To obtain a low-energy state in a translationallyinvariant system when $\Delta$ itself is viewed as a dynamical parameter, we will assume that $\Delta$ is positionindependent, and thus we can still use $\Delta_{\mathbf{k}}$ in the quasiparticle Hamiltonian in $\mathbf{k}$-space. To be consistent with 
this, the fermions must obey either periodic or antiperidic boundary conditions for each of the two primitive directions of the lattice (or fundamental cycles on the torus). For a rectangle with $x$ and $x+L_{x}, y$ and $y+L_{y}$ identified, this means the boundary conditions for the $x$ and $y$ directions. These choices of boundary conditions are well-known in the description of flux quantization in superconductors (see e.g. Schrieffer [2]). We may imagine that either zero or one-half of the flux quantum $h c / e$ threads either of the "holes" (fundamental cycles) in the torus. The half-flux quanta could be represented either by a vector potential, with peridic boundary conditions on the fermions in both directions, or by no vector potential and an antiperiodic boundary condition for each direction that wraps around a flux, or by a combination of these. The different choices are related by gauge transformations. We choose to use boundary conditions and no vector potentials, so that $\Delta$ is always positionindependent. We should be aware that if the gauge field (fluxes) are viewed as external, they are fixed as part of the definition of the problem, and there will be a single ground state for each of the four possible choices of boundary conditions,,,+++--+ , and -- , in a notation that should be obvious. However, if the gauge field is viewed as part of the internal dynamics of the system and can fluctuate quantum mechanically (as in highly correlated systems, including the FQHE, where it is not interpreted as the ordinary electromagnetic field, and also in the usual superconductors where it is) then the four sectors we consider correspond to four ground states of a single physical system, in a single Hilbert space, albeit treated within a mean field approximation. The latter is the view we will take.

For each of the four boundary conditions for the fermions, the allowed $\mathbf{k}$ values run over the usual sets, $k_{x}=2 \pi \nu_{x} / L_{x}$ for,$+ 2 \pi\left(\nu_{x}+1 / 2\right) / L_{x}$ for - , where $\nu_{x}$ is an integer, and similarly for $k_{y}$. In particular, $\mathbf{k}=(0,0)$ is a member of the set of allowed $\mathbf{k}$ only in the case ++ . For a large system, $\xi_{\mathbf{k}}$ and $\Delta_{\mathbf{k}}$ will be essentially the same functions of $\mathbf{k}$ for all four boundary conditions, but evaluated only at the allowed values. In the paired ground states, $\mathbf{k}$ and $-\mathbf{k}$ will be either both occupied or both unoccupied, to take advantage of the pairing $\left(\Delta_{\mathbf{k}}\right)$ term in $K_{\text {eff }}$. When $\mathbf{k}=\mathbf{0}$ is in the set of allowed $\mathbf{k}, \mathbf{k}=\mathbf{0}$ and $-\mathbf{k}=\mathbf{0}$ cannot both be occupied, because of Fermi statistics. However, $\Delta_{\mathbf{k}}$ vanishes at $\mathbf{k}=\mathbf{0}$, so $\mathbf{k}=\mathbf{0}$ will be occupied or not depending solely on the sign of $\xi_{\mathbf{k}=\mathbf{0}}$. That is, it will be occupied for $\mu>0$ (in the weak-pairing phase), and unoccupied for $\mu<0$ (in the strong pairing phase), and this is entirely consistent with the limiting behavior of $\bar{n}_{\mathbf{k}}=\left|v_{\mathbf{k}}\right|^{2}$ as $\mathbf{k} \rightarrow 0$ in the two phases. At the transition, $\mu=0$, the occupied and unoccupied states are degenerate.

We conclude that in either the weak- or strong-pairing phases, there is a total of four ground states, three for boundary conditions,,+--+-- which are linear com- binations of states with even values of the fermion number in both phases, but for ++ boundary conditions the ground state has odd fermion number in the weak-pairing phase, even fermion number in the strong-pairing phase, because of the occupation of the $\mathbf{k}=\mathbf{0}$ state. In most cases, the ground state is as given in Eq. (3), but in the weak pairing phase for ++ boundary conditions, it is

$$
|\Omega\rangle=\prod_{\mathbf{k} \neq \mathbf{0}}^{\prime}\left(u_{\mathbf{k}}+v_{\mathbf{k}} c_{\mathbf{k}}^{\dagger} c_{-\mathbf{k}}^{\dagger}\right) c_{\mathbf{0}}^{\dagger}|0\rangle
$$

The ground states specified, whether for $N$ even or odd, will have the same energy in the thermodynamic limit (not just the same energy density). Note that if the $\mathbf{k}=\mathbf{0}$ state is occupied in the strong-pairing phase, or unoccupied in the weak-pairing phase, this costs an energy $E_{\mathbf{k}=\mathbf{0}}$ which we are assuming is nonzero, and all states where quasiparticles are created on top of our ground states cost a nonzero energy, since we assume that $E_{\mathbf{k}}$ is fully gapped in both phases. However, at the transition $\mu=0$, the ground states for ++ with odd and even particle number are degenerate, and there is a total of five ground states.

If we now compare with results for the MR state on the torus [32,44], which were derived as exact zero-energy ground states of a certain Hamiltonian, then we see that the weak-pairing phase for even fermion number agrees with the exact result that there are three ground states. On the other hand, it was stated in Ref. [44] that there are no zero-energy ground states for $N$ odd. Unfortunately, that result was in error; there is just one such state for ++ boundary conditions, which can be constructed by analogy with that for the 331 state in Ref. 44. Before turning to the wavefunctions of these states, we also mention that the behavior found in the present approach in the strong-pairing phase agrees with that expected in the Halperin point of view [11] on the paired states, as Laughlin states of charge 2 bosons. That point of view predicts four ground states for $N$ even, none for $N$ odd. Note that in comparing with FQHE states, we factor out the center of mass degeneracy which is always the denominator $q$ of the filling factor $\nu=p / q$ (where $p$ and $q$ have no common factors) 64. The remaining degeneracy in a given phase is independent of $\nu$ in the sense that it does not change under the operation of vortex attachment, which maps a state to another in which $1 / \nu$ is increased by 1 , and for generic Hamiltonians this degeneracy is exact only in the thermodynamic limit. We note that Greiter et al. 32] claimed that the special Hamiltonian for which the MR state is exact should have four ground states on the torus for $N$ even, though they found only three. They did not distinguish the weak- and strong-pairing phases, and by assuming that the Halperin point of view is always valid, they in effect ascribed the properties of the strongpairing phase to the MR state. In fact, there is a total of four ground states in the weak-pairing phase, but the 
fourth is at odd fermion number! They also claimed that the statistics would be abelian, even though they considered the MR state, and suggested that the prediction of nonabelian statistics by Moore and Read [14] would hold only at some special point.

If we consider the position-space wavefunctions of the paired states on the torus, then for even $N$ in the weakpairing phase we once again find exact agreement of the long-distance behavior of $g\left(\mathbf{r}_{i}-\mathbf{r}_{j}\right)$ with that which holds for all distances in the eigenstates of the special Hamiltonian [32, 44]. Here long-distance means $g(\mathbf{r})$ where $\mathbf{r}$ is not close to a lattice point $\left(m L_{x}, n L_{y}\right), m, n$ integers. The long-distance form $g \sim 1 / z$ in the plane is replaced by an elliptic function (or ratio of Jacobi theta functions) 32,44 for,,+--+-- . The ground state for $N$ odd with ++ boundary conditions can be written, similarly to one for $N$ even in the 331 case [44], as

$$
\begin{aligned}
\Psi_{++}\left(\mathbf{r}_{1}, \ldots, \mathbf{r}_{N}\right)= & \frac{1}{2^{(N-1) / 2}((N-1) / 2) !} \sum_{P} \operatorname{sgn} P \\
& \times \prod_{i=1}^{(N-1) / 2} g_{++}\left(\mathbf{r}_{P(2 i)}-\mathbf{r}_{P(2 i+1)}\right)
\end{aligned}
$$

Here we can take the torus to have sides $L_{x}, L_{x} \tau$ in the complex plane $\left(\operatorname{Im} \tau>0\right.$, and $\tau=i L_{y} / L_{x}$ for the rectangle), and

$$
g_{++}(\mathbf{r})=\frac{\theta_{1}^{\prime}\left(z / L_{x} \mid \tau\right)}{\theta_{1}\left(z / L_{x} \mid \tau\right)}+\frac{2 \pi i y}{L_{x} \operatorname{Im} \tau}
$$

where $\theta_{1}$ is a Jacobi theta function, and $\theta_{1}^{\prime}(z \mid \tau)=$ $d \theta_{1}(z \mid \tau) / d z . \quad g_{++}(\mathbf{r})$ is periodic because of properties of the function $\theta_{1}$ mentioned for example in Ref. [14], and has a simple pole $g_{++} \propto 1 / z$ as $z \rightarrow 0$. For the ground state with $N$ odd, the non-(complex)-analytic dependence on $y$ in $g_{++}$cancels. Notice that the unpaired fermion with $i=P(1)$ in the terms in $\Psi_{++}$occupies the constant, $\mathbf{k}=\mathbf{0}$ single-particle state. When used as part of a wavefunction in the LLL, the present function is the zero energy state for ++ boundary conditions on the torus for $N$ odd, which was omitted in Ref. 44].

\section{B. Majorana fermions, edges and vortices}

In this Subsection, we consider the problems of edges and of vortices (which correspond to FQHE quasiparticles), on which we argue there are chiral fermions and zero modes, respectively, in the weak-pairing phase. Again, this supports the identification with the MR state.

We begin by considering in more detail the low-energy spectrum near the transition at $\mu=0$. When $\mu$ and $\mathbf{k}$ are small, we can use

$$
\begin{aligned}
\xi_{\mathbf{k}} & \simeq-\mu, \\
\Delta_{\mathbf{k}} & \simeq \hat{\Delta}\left(k_{x}-i k_{y}\right),
\end{aligned}
$$

where $\hat{\Delta}$ can be complex, and find

$$
E_{\mathbf{k}} \simeq \sqrt{|\hat{\Delta}|^{2}|\mathbf{k}|^{2}+\mu^{2}}
$$

which is a relativistic dispersion with $|\hat{\Delta}|$ playing the role of the speed of light. Further, using the same approximation, the BdG equations become in position space

$$
\begin{aligned}
& i \frac{\partial u}{\partial t}=-\mu u+\hat{\Delta}^{*} i\left(\frac{\partial}{\partial x}+i \frac{\partial}{\partial y}\right) v, \\
& i \frac{\partial v}{\partial t}=\mu v+\hat{\Delta} i\left(\frac{\partial}{\partial x}-i \frac{\partial}{\partial y}\right) u,
\end{aligned}
$$

which is a form of the Dirac equation for a spinor $(u, v)$. The BdG equations are compatible with $u(\mathbf{r}, t)=v(\mathbf{r}, t)^{*}$, and this is related to the existence of only a single fermion excitation mode for each $\mathbf{k}$. Thus the quasiparticles are their own antiparticles; Dirac fermions with this property are known as Majorana fermions. Near the transition, the BCS quasiparticles make up a single Majorana fermion quantum field, and at the mean field level the critical theory is a single massless Majorana fermion. There is a diverging length scale at the transition, $\sim \hat{\Delta} /|\mu|$, and the diverging lengths mentioned in the last subsection should all diverge proportionately to this, at least within mean field theory.

Next we wish to consider the behavior near an edge; outside the edge the particle number density should go to zero. In the Hamiltonian, this can be arranged by having a potential $V(\mathbf{r})$ that is large and positive outside the edge. In the quasiparticle effective Hamiltonian, this can be viewed as making $\mu$ large and negative outside the edge, and we will use this notation.

In general, the problem with the edge should be solved self-consistently, which involves solving the gap equation for $\Delta$ in the presence of the edge. We are interested in the generic properties of the solution, and wish to avoid the complexities. Accordingly we will consider only a simplified problem, which is the effective Hamiltonian with a given gap function. Since $\mu$ becomes negative outside the edge, it must change sign near the edge if it is positive inside the bulk of the system. But in the bulk at least, a change in sign across a line represents a domain wall between the weak- and strong-pairing phases, since $\mu=0$ is the point at which the transition occurs in our treatment. Thus we are arguing that the weak-pairing phase (where $\mu$ is positive) must have a domain wall at an edge, while the strong-pairing phase need not. We will consider a domain wall in the bulk as a model for the edge of the weak-pairing phase. In the latter the region of strong pairing between the domain wall and the actual edge may be extremely narrow and we might say there is no well-defined strong-pairing region. But the point is the topological distinction between the phases. The strong-pairing phase has the same topology as the vacuum, and can be continuously connected to it. The 
weak-pairing phase is nontrivial and the generic properties of an edge should be captured by a domain wall 65.

We consider a straight domain wall parallel to the $y$ axis, with $\mu(\mathbf{r})=\mu(x)$ small and positive for $x>0$ and small and negative for $x<0$, and also varying slowly such that the above long-wavelength approximation can be used, with $\mu$ now $x$-dependent.

We can consider solutions with definite $k_{y}$, which at first we set to zero. Then we have a $1+1$-dimensional Dirac equation. We assume that $|\mu| \rightarrow \mu_{0}$, a constant, as $|x| \rightarrow \infty$. At $E=0$ there is a normalizable bound state solution first obtained by Jackiw and Rebbi [66]. The equations are (with $\hat{\Delta}$ real and $>0$ )

$$
\begin{aligned}
& \hat{\Delta} i \partial v / \partial x=\mu(x) u \\
& \hat{\Delta} i \partial u / \partial x=-\mu(x) v .
\end{aligned}
$$

By putting $v=i u$, we find the unique normalizable solution

$$
u(x) \propto e^{-i \pi / 4} \exp -\frac{1}{\hat{\Delta}} \int^{x} \mu(x) d x,
$$

where the phase was inserted to retain $v=u^{*}$. Solutions at finite $E$ should exhibit a gap, as in the bulk in either phase.

At finite $k_{y}$, the equations become

$$
\begin{aligned}
& E u=-\mu u+\hat{\Delta} i\left(\frac{\partial v}{\partial x}-k_{y} v\right), \\
& E v=\mu u+\hat{\Delta} i\left(\frac{\partial u}{\partial x}+k_{y} u\right) .
\end{aligned}
$$

For $E=-\hat{\Delta} k_{y}$, these have solutions that are bound to the domain wall, and clearly they propagate in one direction along the wall. There is only one fermion mode for each $k_{y}$, and so we have a chiral Majorana (or MajoranaWeyl) fermion field on the domain wall.

It will be important to consider also a pair of domain walls. We consider two walls, lying at $x=0$ and $x=W$, with $\mu>0$ in $0<x<W$ and $\mu<0$ outside. Again we assume $k_{y}=0$ initially. This time [66] there are no $E=0$ modes for finite $W$. Clearly as $W \rightarrow \infty$ we expect to find an $E=0$ mode on either wall, so we expect bound solutions for small $E$ when $W$ is large but finite. For nonzero $E$ we can replace the pair of first-order equations with a single second-order equation for either of $u \pm i v$, (with $k_{y} \neq 0$ for generality),

$$
\left(E^{2}-\hat{\Delta}^{2} k_{y}^{2}\right)(u \pm i v)=\left(-\hat{\Delta}^{2} \frac{\partial^{2}}{\partial x^{2}}+\mu^{2} \pm \hat{\Delta} \frac{\partial \mu}{\partial x}\right)(u \pm i v) .
$$

When $\mu$ varies slowly compared with its magnitude $\mu_{0}$ far from the walls, we may study the equations by the
WKB method. We may view the equation for $u \pm i v$ as a Schrodinger equation with potential

$$
V_{ \pm}(x)=\mu^{2} \pm \hat{\Delta} \frac{\partial \mu}{\partial x}
$$

If $\partial \mu / \partial x$ has extrema at $x=0, W$, as is reasonable, then $V_{-}(x)$ has minima at $x=0, W$, but that at $x=0$ is deeper than that at $x=W$. The reverse is true for $V_{+}(x)$. As $W \rightarrow \infty$, there will be a $k_{y}=0$ solution for $E^{2}$ which $\rightarrow 0$ exponentially and which corresponds to a normalizable eigenfunction for $u-i v$ that is concentrated at $x=0$ with negligible weight at $x=W$, and similarly an eigenfunction for $u+i v$ concentrated at $x=W$. The subtle but important point is that these solutions are not independent, because they are related by the original first-order system, for any non-zero $E$. There is only a single normalizable solution for the pair $u, v$ for $E$ small positive, and another for $E<0$. Consequently, there is only a single fermion mode, shared between the two domain walls, not one on each. For non-zero $k_{y}$, the eigenfunctions for $E>0$ become concentrated on one wall or the other, depending on the sign of $k_{y}$. Thus the set of low-energy states can be viewed as a single non-chiral Majorana fermion theory, with the left-moving modes on one wall, the right-moving modes on the other, and the $k_{y}=0$ mode shared between the two. This agrees precisely with the results of Ref. [42] on the edge states of the MR state on the cylinder.

We next consider the quasiparticle spectrum in the presence of vortices of the order parameter; in two dimensions vortices are point objects. These necessarily contain an integer number of half flux quanta in the gauge field; without the gauge field the vorticity is quantized but the total energy of an isolated vortex diverges logarithmically. We will not see these effects in the energy here because we only consider the quasiparticle excitation spectrum in the presence of a given gap function and gauge field configuration. Up to now we have ignored the $\mathrm{U}(1)$ gauge field except in discussing the boundary conditions on the torus and cylinder. It could be the standard electromagnetic field in a superconductor, or the CS field in the FQHE. We will only consider vortices of the minimal flux, namely a half flux quantum, because addition of any integer number of flux quanta can be viewed, on scales larger than the penetration depth, as a gauge transformation, which does not affect the spectrum. Outside the vortex core, which we assume is small, the covariant derivative of the gap function must vanish. As in the case of the torus, we will choose a gauge in which the gap function is single-valued and independent of the angle relative to the position of the nearby vortex, but the Fermi fields are double-valued on going around the vortex.

The basic idea is to consider a vortex as a small circular edge, with vacuum (vanishing density) at the center. Accordingly, we expect that nothing interesting happens at 
sufficiently low energies for vortices in the strong-pairing phase. But a vortex in the weak-pairing phase must include a concentric circular domain wall to separate the vacuum at the center from the weak-pairing phase outside. We now study this using the Majorana fermion equations near the transition, assuming that the wall has large enough radius; the vortex core (where $\Delta$ vanishes) can be taken to have negligible size and the boundary condition at $r \rightarrow 0$ is unimportant. With our choice, the $\mathrm{BdG}$ equations for a single vortex and for $E=0$ becomes in polar coordinates $r, \theta$

$$
\begin{aligned}
\hat{\Delta} i e^{i \theta}\left(\frac{\partial}{\partial r}+\frac{i}{r} \frac{\partial}{\partial \theta}\right) v & =\mu u \\
\hat{\Delta} i e^{-i \theta}\left(\frac{\partial}{\partial r}-\frac{i}{r} \frac{\partial}{\partial \theta}\right) u & =-\mu v ;
\end{aligned}
$$

$u$ obeys $u(r, \theta+2 \pi)=-u(r, \theta)$, and similarly for $v$. We can assume $\mu \rightarrow \mu_{0}>0$ as $r \rightarrow \infty, \mu \rightarrow-\mu_{0}$ as $r \rightarrow 0$. The normalizable solutions have the form

$$
\begin{aligned}
& u=(i \bar{z})^{-1 / 2} f(r), \\
& v=(-i z)^{-1 / 2} f(r)=u^{*},
\end{aligned}
$$

where $f(r)$ is a real function. The equations reduce to

$$
d f / d r=-\mu(r) f(r) / \hat{\Delta},
$$

with solution

$$
f(r) \propto \exp \left(-\int^{r} \mu\left(r^{\prime}\right) d r^{\prime} / \hat{\Delta}\right) .
$$

Thus we find just one normalizable bound state at zero energy. Again we expect this to persist as we relax our assumptions, as long as the bulk outside the vortex is in the weak-pairing phase. We point out that our result should be contrasted with the known result for a vortex in an s-wave superconductor, which has bound quasiparticle modes at energies that are low in the weak coupling limit, but not generally zero as ours are here [67]. Since we mainly work at moderate or strong coupling, analogous modes are not important for our purposes. We note that a zero mode on a vortex in an A-phase p-wave paired state was first found in Ref. [68].

For the case of $2 n$ well-separated vortices, we have not obtained analytic solutions for the bound states. However, we can give a simple argument. The problem is analogous to a double-well potential. We take a set of $2 n E=0$ solutions like Eqs. (30), (32) centered at each vortex, and use these as a basis set (we must introduce additional branch points into each basis state to satisfy the boundary conditions at all the other vortices); at finite separation there is mixing of the states, and the energies split away from zero. Since the solutions to the Dirac equation are either zero modes or $E,-E$ pairs, we expect to obtain $n E>0$ solutions, $n E<0$ solutions. In general, each $E>0$ solution of the Dirac or BdG equation corresponds to a creation operator, and the related $E<0$ solution to the adjoint (destruction) operator, while an $E=0$ solution would correspond to a real (or Majorana) fermion operator. In our case, this means that there are $n$ modes in which we may create fermions, with energies $E$ tending to zero as the separation diverges. (A similar picture applies for $2 n$ domain walls.) This is in agreement with the results for the special Hamiltonian 44]. This result is crucial for the nonabelian statistics we expect in the FQHE case, since by occupying the zero modes one obtains a total of $2^{n}$ degenerate states, or $2^{n-1}$ for either even or odd fermion number $N$, when there are $2 n$ vortices $(n>0)$; this was found for the special Hamiltonian in Ref. 43, 44].

We may also consider here the edge states of a system in the form of a disk of radius $R$, by studying a large circular domain wall enclosing the weak-pairing phase, and strong-pairing phase or vacuum outside. In this case, there is no flux enclosed by the wall, and $u$ and $v$ are single-valued. One does not find $E=0$ states, but instead there is a set of chiral fermion modes with angular momentum $m$ quantized to half-integral values, $m \in \mathbf{Z}+1 / 2$, and $E \propto m / R$ (this fixes the definition of $m=0$ ). These are just the modes expected for the chiral Majorana fermion on such a domain wall with the ground state inside, since an antiperiodic boundary condition is natural for the ground state sector. If a half-flux quantum is added at the center of the disk, the quantization is $m \in \mathbf{Z}$, and this extends the result for the zero mode $m=0$ of a single vortex. These results agree with the results of Ref. 49, 49 for a disk of the MR state.

We also note that the form of the modes near a vortex, containing $z^{-1 / 2}$ or its conjugate, is similar to the form of the fermion zero mode functions found in Ref. [44], once the factors associated with the charge sector are removed, though the factors $f(r)$ are not. However, the most appropriate comparison to make is that between the many-fermion wavefunctions, as we already made for the ground states on the plane and torus. We will not consider this further here for the vortex or the edge (or domain wall) states, though we expect that these should correspond at long distances to those found in Ref. 43, 44, 42, as for the ground states. However, we are able to find the ground states on other geometries, namely the sphere and Riemann surfaces of genus (number of handles) greater than one (the sphere is genus zero, the torus genus one). We consider this briefly in Subsection III below.

\section{Other geometries and conformal field theory}

In this Subsection, we briefly introduce some general connections of pairing theory for p-wave states to rela- 
tivistic fermions, which enables us to discuss geometries other than the plane and torus, such as the sphere, and to make more explicit connections with conformal field theory ideas [14. This Subsection can be omitted on a first reading, but some of the formalism is mentioned again later.

In the preceding Subsection, we used the fact that the $\mathrm{BdG}$ equations at long wavelengths become the Dirac equation, with a reality condition so that the Fermi field is a Majorana fermion. We also mentioned the coupling of the gap function and Fermi fields to a $\mathrm{U}(1)$ vector potential (which in the FQHE context would be the CS vector potential), which is of a standard form. But the interpretation of the fermion as Majorana would seem to raise a problem, because for a single Majorana there is no continuous symmetry of the Yang-Mills type, and so apparently no way to minimally couple it to a vector potential. We will see that there is nonetheless a natural way to incorporate the vector potential and still give an interpretation in terms of the Dirac equation, and this will also enable us to discuss the ground states on curved surfaces.

The most general form for the p-wave gap function in Fourier space, retaining once again only the longwavelength part, can be written

$$
\Delta_{\mathbf{k}}=\Delta_{x} k_{x}-i \Delta_{y} k_{y} .
$$

Here $\Delta_{x, y}$ are two complex coefficients, or equivalently four real numbers, which we will arrange into a $2 \times 2$ matrix $e$. In position space, the $\mathbf{k}$ can be replaced by $-i \nabla$. Then in a general coordinate system $x^{i}$, with corresponding partial derivatives $\partial_{i},(i=x, y)$, the $\mathrm{BdG}$ equations become

$$
\left(\partial_{t}+\frac{1}{2} i \omega_{t}^{b c} \Sigma_{b c}\right) \psi+e^{i a} \alpha_{a}\left(\partial_{i}+\frac{1}{2} i \omega_{i}^{b c} \Sigma_{b c}\right) \psi+i \beta m \psi=0,
$$

where we use a spinor $\psi=(v, u)$, and $m=\mu$ in previous notation. The indices $a, b, c$ take the values $x, y$, and the matrices are $\alpha_{x}=\sigma_{x}, \alpha_{y}=\sigma_{y}, \beta=\sigma_{z}$; we use the summation convention. Here we have also reinstated the vector potential $A_{\mu}=\omega_{\mu}^{x y} / 2$ (where $\mu=t, x, y$ ), using the matrix

$$
\Sigma_{x y}=\sigma_{z} .
$$

The equation is therefore invariant under $\mathrm{U}(1)$ gauge transformations $\psi \rightarrow e^{i \Lambda^{x y} \Sigma_{x y}} \psi$, and a corresponding transformation of $\omega_{\mu}$, with a real scalar parameter $\Lambda_{x y}\left(x^{\mu}\right)$.

If we multiply through by $\beta$, then we obtain the more covariant form of the Dirac equation,

$$
e^{\mu a} \gamma_{a}\left(\partial_{\mu}+\frac{1}{2} \omega_{\mu}^{b c} \Sigma_{b c}\right) \psi+i m \psi=0,
$$

with $e^{\mu t}=\delta_{\mu t}$ in our case. This has the form of the general Dirac equation suitable for use in general coordinate systems on general curved spaces or spacetimes [69]. A similar form was obtained in Ref. [7]. In general, $\psi$ is a Dirac spinor (with two components in the 2 and $2+1$ cases of interest here); $\mu=1,2, \ldots d$ is a spacetime index, while $a, b, c=1,2, \ldots, d$ is an internal "local Lorentz" index; the vielbein $e$ is a tensor with indices as shown; $\gamma_{a}$ are a set of Dirac matrices satisfying $\left\{\gamma_{a}, \gamma_{b}\right\}=2 \eta_{a b}$, where $\eta$ is the Minkowski or Euclidean metric, and $\Sigma_{a b}=\frac{1}{2} i\left[\gamma_{a}, \gamma_{b}\right]$ are the generators of $\mathrm{SO}(d-1,1)$ Lorentz transformations (or simply $\mathrm{SO}(d)$ rotations, in the Euclidean case); the spin connection $\omega$ is a tensor field with one spacetime and two internal Lorentz indices, and is antisymmetric in the latter. Spacetime indices are raised and lowered using $g^{\mu \nu}$ and $g_{\mu \nu}$, while internal Lorentz indices are raised and lowered using $\eta^{a b}$ and $\eta_{a b}$. This form of the Dirac equation is covariant under coordinate transformations (diffeomorphisms), under which the spinor is viewed as transforming as a scalar function of position, and $e$ and $\omega$ as tensors. It is also covariant under $\mathrm{SO}(d-1,1)$ (or $\mathrm{SO}(d))$ local Lorentz transformations, which act like gauge transformations, with $\psi$ transforming in the spinor representation, the vielbein transforming as a vector in the $a$ index, and $\omega$ transforming inhomogeneously as a nonabelian vector potential or connection for the gauge transformations. This formalism (also known, in four dimensions, as the vierbein or tetrad formalism) can be used to reformulate, for example, general relativity in a form equivalent to the usual one involving Christoffel symbols; this involves imposing relations

$$
\begin{aligned}
e_{\mu}^{a} e_{\nu a} & =g_{\mu \nu}, \\
e_{a}^{\mu} e_{\mu b} & =\eta_{a b} .
\end{aligned}
$$

On the other hand, it is the only known way to couple Dirac fields to curved spacetime; more details can be found in Ref. 69. In our case, we have a distinguished choice of time coordinate, we consider only the restricted form with $e^{\mu t}=\delta_{\mu t}$, and require covariance under only the $\mathrm{SO}(2) \cong \mathrm{U}(1)$ subgroup that describes "internal spatial rotations". Then the Dirac equation becomes precisely the BdG equation. This relation with the vielbein formalism suggests that the vector potential will play a natural role when we consider pairing of nonrelativistic fermions on a curved surface.

The problem of spinless p-wave pairing of nonrelativistic fermions moving on a general curved manifold should be formulated as follows. The manifold has a metric and a corresponding Riemann curvature tensor, which for two dimensions reduces to a curvature scalar. We will consider only the case in which this curvature is constant on the manifold, and we will also introduce a U(1) or $\mathrm{SO}(2)$ gauge potential. For a large system, the radius of curvature of the manifold is large, and locally the so- 
lution to the gap equation should resemble that in flat space, which we assume is of the $l=-1$ form. In order to minimise the energy density, we expect that the gap function should be as constant as possible. In more invariant language, this means that the vielbein should be covariantly constant (note that the covariant derivative of the vielbein must be covariant under both coordinate and U(1) gauge transformations), and there should be no vortices. Since the Riemannian geometry (the metric and the Levi-Civita or metric connection) of the manifold are assumed given, this condition relates the spin connection $\omega$ to derivatives of the vielbein, a result analogous to the usual requirement (for s-wave pairing, in flat space) that the vector potential be the gradient of the phase of the gap function. This covariant-constancy condition also appears when formulating general relativity [69].

This condition can be satisfied globally (we assume the surface on which we are working is compact) only if the field strength in the $\mathrm{SO}(2)$ vector potential is related to the Riemann curvature of the manifold. The integral of the latter over the surface, divided by $4 \pi$, is a topological invariant, the Euler invariant, equal to $2(1-g)$ for a Riemann surface of genus $g$ (one with $g$ handles). In our usual units for flux quanta, the number of flux quanta in the $\mathrm{SO}(2)$ or $\mathrm{U}(1)$ vector potential must be $g-1$. Otherwise, we will have vortices somewhere on the surface, at which $\hat{\Delta}$ goes to zero. In particular, for the sphere, this agrees with the familiar fact for the MR state that the number of flux $N_{\phi}$ seen by the underlying particles is one less than in the Laughlin state at the same filling factor, so the composite fermions see a net flux of -1 . Physically, the nonzero angular momentum of the pairs causes them to see the curvature of the manifold on which they move as a gauge field, the field strength of which is cancelled (locally, not just globally) by the imposed $\mathrm{U}(1)$ (i.e. $\mathrm{SO}(2)$ ) gauge field, so that a uniform condensate is possible, much like the condition of vanishing field strength for uniform s-wave condensates in ordinary superconductors.

It can be shown that the long-wavelength wavefunction involves the inverse of (or Green's function for) part of the (covariant) Dirac operator we have discussed, namely the part $\Delta^{\dagger}$, where $\Delta$ is the part of the Dirac operator, including the vector potential, that acts on $c^{\dagger}$ and maps it to $c$ (like the earlier gap function) in the Dirac equation. $\Delta^{\dagger}$ contains derivatives like $\partial / \partial \bar{z}$ in local coordinates. On the sphere, in stereographic coordinates the Green's function is known to be essentially $1 /\left(z_{i}-z_{j}\right)$ for particles $i$ and $j$. For any surface, this description in terms of inverting the massless Dirac operator is identical to the problem of finding the correlators of two-dimensional chiral Majorana fermions (in Euclidean spacetime), and so it is not surprising that this agrees with the conformal block for $N$ two-dimensional massless fermions on the sphere in conformal field theory. We note that the paired ground state on the sphere can be described in angular momen- tum space, in terms of single-particle angular-momentum eigenstates with eigenvalues $j, m$ (and $j=1 / 2,3 / 2, \ldots$, due to the single flux quantum), as BCS pairing of $j, m$ with $j,-m$; antisymmetry and vanishing total angular momentum for each pair require that the $j$ 's be half-oddintegral, as they are for p-wave.

We may also consider the cases of Riemann surfaces of genus greater than one. Here the explicit functions, which again are built out of $1 / \Delta^{\dagger}$, are more difficult to find, but certainly exist and describe the MR state on these surfaces. The required number of flux seen by the fermions is $g-1$. The only aspect we wish to discuss further here is the number of distinct ground states for $g>1$. When handles are present, the vector potential is determined only up to addition of a pure gauge piece describing holonomy around the $2 g$ fundamental cycles of the surface. The holonomy, or phase picked up when a fermion is parallel-transported around a cycle, can only be \pm 1 , since it comes from the double cover of $\mathrm{SO}(2)$ by $\operatorname{Spin}(2)$, the group which possesses the spinor representation. This effect, which is a restatement of flux quantization, agrees with and generalizes the discussion of boundary condition sectors for the torus. There is thus a set of $2^{2 g}$ possible boundary condition sectors, which in the present differential geometry set-up are known as spin structures. The spin structures on a genus $g$ surface can be divided into two sets, known as the even and odd spin structures. The difference between these, for our purposes, is that the odd spin structures possess a single zero mode for the Dirac operator, and the even spin structures possess none. Then the BCS ground states in the weak-pairing phase will include one fermion occupying the zero mode when one exists, and since the other fermions are all paired, we conclude that the odd spin structures give rise to ground states with $N$ odd, and the even spin structures to $N$ even, and these ground states will be degenerate in the thermodynamic limit. It is known that there are $2^{g-1}\left(2^{g}+1\right)$ even spin structures, and $2^{g-1}\left(2^{g}-1\right)$ odd spin structures, so these formulas give the number of ground states for $N$ even and odd, for all $g \geq 0$. These numbers (and the long-distance wavefunctions) agree with the conformal blocks for a correlator on the genus $g$ surface with $N$ Majorana fields inserted. All of this is in beautiful agreement with the CFT picture of Ref. [14]. We note that the U(1) charge sector which is present in the FQHE states gives another factor $q^{g}$ in the degeneracy for filling factor $\nu=p / q$, in the thermodynamic limit [70], which generalizes the center-of-mass degeneracy $q$ of the torus [64].

\section{SPIN-TRIPLET COMPLEX P-WAVE PAIRING}

In this Section we consider spin-triplet p-wave pairing. Since the general classification of such states is compli- 
cated (compare the three-dimensional version in Ref. [3]), we concentrate on a particular case directly related to the FQHE. The FQHE system we have in mind is the double-layer system at $\nu=1 / 2$ 37]. This is assumed to be spin-polarized, but the layer index of the electrons plays the role of a spin, which we refer to as isospin, to avoid confusion with the pseudospin discussed in the previous Section. The $S_{z}$-values of the isospin will be denoted $\uparrow, \downarrow$ for the two layers.

In the double-layer FQHE system, as in the other systems we discuss, we go to a CS fermion representation by using layer-independent fluxes attached to the CS fermions. Because interactions between electrons in the same and in different layers are different (though the two layers are on an equal footing), the Hamiltonian will not have SU(2) symmetry. However, in the absence of a tunneling term and of interactions that transfer electrons between layers, the number $N_{\uparrow}-N_{\downarrow}$ is conserved, and since this quantity is twice the total $S_{z}$ of the isospin, there is a $\mathrm{U}(1) \cong \mathrm{SO}(2)$ symmetry that rotates the isospin about the $z$ axis. Also interchange of the two layers (or reflection in the plane midway between the two layers) is a $\mathbf{Z}_{2}$ symmetry. Together these make up an $\mathrm{O}(2)$ symmetry. We also consider the effect of a tunneling term $-t \sigma_{x}$ for each particle; $t$ is the tunneling amplitude and $\sigma_{x}$, etc, denote the Pauli matrices. Nonzero $t$ breaks the symmetry to the $\mathbf{Z}_{2}$ of layer exchange.

The FQHE system at $\nu=1 / 2$ has a possible ground state which is a 331 state, which can be viewed as complex p-wave pairing of composite fermions 44. The pairing and the effective quasiparticle Hamiltonian are best considered in terms of isospin states which are eigenstates of $\sigma_{x}$, namely $e=(\uparrow+\downarrow) / \sqrt{2}, o=(\uparrow-\downarrow) / \sqrt{2}$, which are respectively even, odd under the $\mathbf{Z}_{2}$. As in the earlier work on this problem, we assume that the $\mathbf{Z}_{2}$ symmetry, and for $t=0$ the $\mathrm{O}(2)$ symmetry, are not broken spontaneously. Then symmetry dictates that the effective quasiparticle Hamiltonian has the form

$$
\begin{aligned}
K_{\mathrm{eff}}= & \sum_{\mathbf{k}}\left[\left(\xi_{\mathbf{k}}-t\right) c_{\mathbf{k} e}^{\dagger} c_{\mathbf{k} e}+\frac{1}{2}\left(\Delta_{\mathbf{k} e}^{*} c_{-\mathbf{k} e} c_{\mathbf{k} e}\right.\right. \\
& \left.+\Delta_{\mathbf{k} e} c_{\mathbf{k} e}^{\dagger} c_{-\mathbf{k} e}^{\dagger}\right)+\left(\xi_{\mathbf{k}}+t\right) c_{\mathbf{k} o}^{\dagger} c_{\mathbf{k} o} \\
& \left.+\frac{1}{2}\left(\Delta_{\mathbf{k} o}^{*} c_{-\mathbf{k} o} c_{\mathbf{k} o}+\Delta_{\mathbf{k} o} c_{\mathbf{k} o}^{\dagger} c_{-\mathbf{k} o}^{\dagger}\right)\right]
\end{aligned}
$$

We have taken the same kinetic term $\xi_{\mathbf{k}} \simeq k^{2} / 2 m^{*}-\mu$ for both $e$ and $o$ since a difference here is unimportant (and forbidden by symmetry when $t=0$ ). For $t=0$, $\Delta_{\mathbf{k} e}=\Delta_{\mathbf{k} o}$, and in general we assume both have p-wave symmetry, with

$$
\Delta_{\mathbf{k} e} \simeq \hat{\Delta}_{e}\left(k_{x}-i k_{y}\right)
$$

at small $\mathbf{k}$, and similarly for $\Delta_{\mathbf{k} o}$. We have also neglected the possibility of many-body renormalization of the split- ting $2 t$ between $e$ and $o$ (such as an exchange enhancement).

We see that the unbroken $\mathbf{Z}_{2}$ symmetry has led to decoupled $e$ and $o$ Hamiltonians. These are the same as for the spinless p-wave case. Consequently, we see that separate transitions from weak to strong pairing are possible when $t \neq 0$. For $t=0$, these coincide. The pairing function $g(\mathbf{r})$ in the wavefunction is now a four-component object because of the isospin variables. We write it as a vector in the tensor product space of the two spinors. When at least one of the two components is in its weakpairing phase, the pairing function at long distances has the form

$$
\frac{\cos (\theta-\pi / 4) e_{i} e_{j}+\sin (\theta-\pi / 4) o_{i} o_{j}}{z_{i}-z_{j}} .
$$

This is the form that was assumed for all distances in Refs. 36,71,44. For $t=0$, we would put $\theta=0$, in which case it reduces to

$$
\frac{\uparrow_{i \downarrow_{j}}+\downarrow_{i} \downarrow_{j}}{z_{i}-z_{j}},
$$

which is the form in the 331 state. As $t$ increases, neglecting the likely change in $\Delta_{\mathbf{k} e}, \Delta_{\mathbf{k} o}$ momentarily, a point is reached at which the $o$ spins have an effective chemical potential $\mu-t=0$, and undergo a transition to strong pairing. Then the long distance behavior is $e_{i} e_{j} /\left(z_{i}-z_{j}\right)$, so $\theta=\pi / 4$ at the transition and remains at that value thereafter. The resulting phase is expected to have the statistics properties of the MR state, unaffected by the strong-pairing oo pairs present in the ground state.

When $\mu$ is decreased, there will be a transition from the MR phase to strong pairing in both components when $\mu+t=0$. Thus we obtain the phase diagram shown in Fig. 1. We have also included labels of the analogous phases in $\mathrm{He}^{3}$ [3]. In $\mathrm{He}^{3}$, the roles of $e, o$ are played by $\uparrow, \downarrow$, and that of $t$ is played by the Zeeman splitting due to a field $h$ along the $z$ direction. In $\mathrm{He}^{3}$, there is full $\mathrm{SU}(2)$ symmetry of spin rotations when $h=0$, that is broken spontaneously in any spin-triplet phase, but this distinction is unimportant here. The state for $t=0$ has the structure of the ABM state or A phase, adapted to two dimensions, while for $t \neq 0$, we expect that self-consistent solution of the gap equation would give $\hat{\Delta}_{e}>\hat{\Delta}_{o}$, and this state has the structure of the A2 phase of $\mathrm{He}^{3}$. As $t$ increases, a point may be reached at which $\Delta_{\mathbf{k} o}=0$ for all $\mathbf{k}$, which gives the A1 phase (we ignore intermediate possibilities in which $\Delta_{\mathbf{k} o}$ vanishes only in some region of $\mathbf{k}$ space). For $\mu-t>0$, which would be the case in $\mathrm{He}^{3}$, the $\mathrm{A} 1$ phase is a distinct phase, which would have a Fermi surface for $o$ spins. On the other hand, for $\mu-t<0$, no excitations become gapless at the point where $\Delta_{\mathbf{k} o}$ vanishes, and the change is merely the disappearance of oo pairs from the ground state, so this is not a true phase transition; this is indicated by 


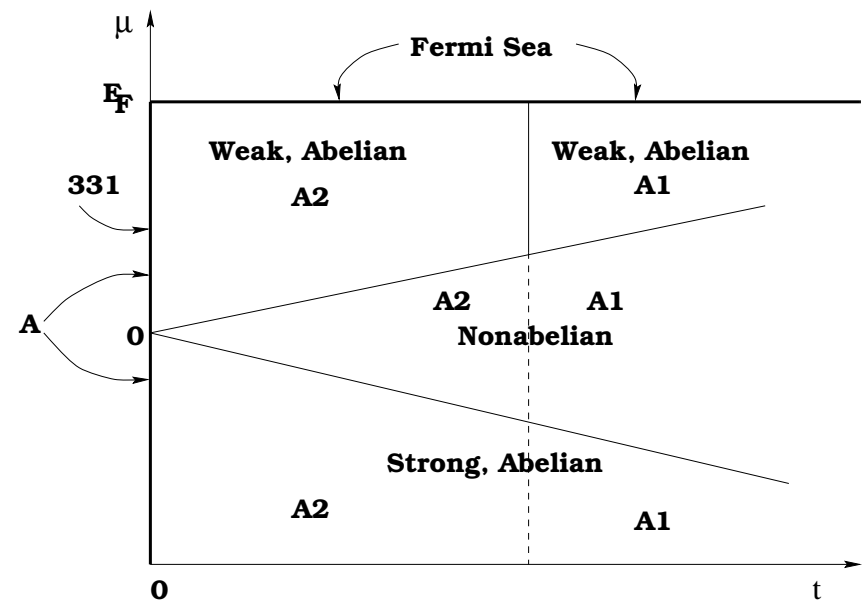

FIG. 1. Schematic phase diagram for the p-wave phases, as discussed in the text. The A-phase with unbroken $\mathrm{U}(1)$ symmetry appears as the vertical axis $t=0$, with the region $\mu>0$ being the 331 phase. Similarly, the Fermi-liquid phase in which pairing disappears is identified with the line $\mu=E_{F}$, since that is the value of $\mu$ there at fixed density, neglecting Hartree-Fock corrections.

the dashed line in Fig. 1. The position of this boundary, if it occurs at all, is very uncertain. We simply wish to emphasize that the boundaries between A, A2 and A1 in general do not coincide with the weak- to strongpairing transitions. However, for the wavefunctions that are of the form Pf $g$ with $g$ given by Eq. (40) for all distances, the transition at $\theta=\pi / 4$ to the MR state can also be considered as the A2-A1 transition. We discuss this further below.

For $t=0$, the quasiparticle excitations near $\mathbf{k}=0$ and $\mu \simeq 0$ form a Dirac relativistic fermion spectrum, which has two distinct but degenerate particle and antiparticle excitations. These are eigenstates of the U(1) symmetry of eigenvalues $+1,-1$, respectively, so the particle is associated with one layer, the antiparticle with the other. A Dirac fermion field is equivalent to two Majorana fields, which can be thought of roughly as its real and imaginary components. For $t \neq 0$, these Majorana fermions have different masses (and different velocities if $\hat{\Delta}_{e} \neq \hat{\Delta}_{o}$ ), and the transitions occur when one or other mass changes sign.

The ground-state degeneracies, edge state and vortex (quasiparticle) properties in the different phases can now be read off from the above and the results for the spinless p-wave case. For $\mu>0$ and $t=0$, we find the same results as for the 331 state 42,44]. The FQHE state is abelian and can be described in Coulomb plasma language because of the Cauchy determinant identity, as explained in Ref. 42, 44. The equivalence of the results for the neutral edge excitations, or for the quasihole states, in terms of bosonic fields, or the two-component plasma mapping, and Dirac fermion fields, corresponds to bosonization [42,44] and will not be repeated here in full. We will mention only an instructive example, consisting of a pair of vortices in the weak pairing phase $\mu>0$. Given that there is a single $E=0$ mode for the pair, which can here be occupied by either type of fermion, then there are four states. Since the fermions in the $\uparrow-\downarrow$ basis have $S_{z}$ quantum numbers $\pm 1 / 2$, the four states have $S_{z}$ values $1 / 2,0,0,-1 / 2$ (half of these states have odd total fermion number $N$ ). These states must be interpreted as saying that each of these elementary vortices carries $S_{z}$ of $\pm 1 / 4$, so there is a fractionalization of the $S_{z}$ quantum number. For $N$ even we have only two states, both with $S_{z}=0$, if there are no other excitations of the system, due to global selection rules related to the total quantum numbers. This agrees with the two-component plasma description for the 331 states, on including the charge degree of freedom in the incompressible FHQE system [42, 44. In the latter formulation, the fractional $S_{z}$ is analogous to the fractional charge of the Laughlin quasiparticles.

For $t \neq 0$, the $\mathrm{U}(1)$ symmetry is lost, and the quantum field theories, whether the massive theory in the bulk or the chiral theory on the edge or at the vortices, in the phase labelled "weak-pairing abelian" must be described as two Majorana fermions. However, the counting of the edge excitations, or of the vortex states just discussed, will be the same (though unimportant degeneracies among edge excitations, that previously were due to the U(1) symmetry, may be lost), and the universal statistics properties in this phase, which are abelian, are the same as in the U(1) symmetric or Dirac fermion case. As already mentioned, when the transition to strongpairing occurs for the $o$ spins, the system enters the MR nonabelian phase, and when the $e$ spins are also in the strong-pairing phase, the FQHE system has the rather trivial abelian statistics of the Laughlin state of charge 2 bosons.

Ideas of Ho [71 involving a two-body pseudopotential Hamiltonian, the ground state of which interpolates between the 331 state at $\theta=0$ and the MR state at $\theta=\pi / 4$ were discussed critically in Ref. 444. The model Hamiltonian contains a parameter corresponding to $\theta$ which directly determines the $\theta$ that describes the ground state wavfunction. Except at $\theta=\pi / 4$, the results of the model agree with the above discussion of the abelian phase, though we do not now believe that the U(1) symmetry is "reappearing in the low-energy properties" 44]. At $\theta=\pi / 4$, Ho's model is pathological, and the upshot of the discussion was that if the three-body interaction of Greiter et al. is also added as a isospin-independent interaction, then this pathology of Ho's model is removed, and the ground state is nondegenerate (on the sphere, without quasiholes) for all $\theta$ between 0 and $\pi / 4$. It was noted that, even for this model, there are still peculiarities of the $\theta=\pi / 4$ point. In fact, there are unexpected degeneracies, larger than those of the 331 or $0 \leq \theta<\pi / 4$ states, in the edge, quasihole and toroidal ground states 
at $\theta=\pi / 4$. These arise because the zero modes can be occupied by either $e$ or $o$ fermions, even though there are no os in the ground state. In particular, on the torus in the ++ sector, there are degenerate states that differ only in the presence or absence of a $\mathbf{k}=0 o$ fermion (these extra degeneracies in this model at this point were overlooked in Ref. 44, but can be obtained by the same methods used there). This clearly suggests that the dispersion relation $E_{\mathbf{k} o}$ for the $o$ Majorana fermions is gapless at this point, and seems to confirm that this model at $\theta=\pi / 4$ is actually at the transition point between the weak-pairing abelian and MR nonabelian phases. This is consistent with the result of the analysis here that when the leading long-distance part of $g$ is described by $\theta=\pi / 4$, the system is either at the transition or in the MR phase. This is certainly not the case for $\theta<\pi / 4$, as indicated by the degeneracies found for the Ho Hamiltonian plus three-body in Ref. [44. However, the fact that the ground state of the model at $\theta=\pi / 4$ contains no $o$ fermion pairs at all, suggests that this point is at the A2A1 boundary as well as at the weak-MR transition. In the quasiparticle effective Hamiltonian, this would correspond to vanishing $\Delta_{o}$ as well as $\mu$, and therefore we would expect the dispersion relation for the $o$ fermions to be $E_{\mathbf{k}} \propto|\mathbf{k}|^{2}$. Clearly this is nongeneric behavior. When tunneling $t$ is also included, the value of $\theta$ in the ground state cannot be read off the Hamiltonian in general, but we expect that the weak to MR transition, which should now be generic, is pushed to another value of the parameter in the Hamiltonian, so the region in the MR phase with $\theta=\pi / 4$ widens, while the A2-A1 boundary is still at the value corresponding to $\theta=\pi / 4$ originally, where the ground state is known exactly 44, and again contains no $o$ fermions.

\section{COMPLEX D-WAVE SPIN-SINGLET PAIRING}

In this Section we consider $l=-2$ complex d-wave pairing of fermions, and more generally $\mathrm{d}_{x^{2}-y^{2}}+i \mathrm{~d}_{x y}$, which are necessarily spin-singlet. This has been considered recently [59,55 58]. We argue that the HaldaneRezayi (HR) state [13], which has this symmetry, is at the transition from weak to strong pairing. The weak pairing phase, like the strong, is abelian; we work out its properties and its lattice description from the pairing point of view, and identify the universality class as one which has been obtained before by various methods, including a trial wavefunction approach by Jain [22,72 74. We relate these properties to the Hall conductivity for spin, which we calculate explicitly in an Appendix.

\section{A. Weak- and strong-pairing phases, and the Haldane-Rezayi FQHE state}

The basic structure of the problem is once again similar to the spinless p-wave case, or (since it is spin-singlet) to the original BCS treatment, except for being d-wave. The quasiparticle effective Hamiltonian is

$K_{\mathrm{eff}}=\sum_{\mathbf{k} \sigma}\left(\xi_{\mathbf{k}} c_{\mathbf{k} \sigma}^{\dagger} c_{\mathbf{k} \sigma}+\Delta_{\mathbf{k}}^{*} c_{-\mathbf{k} \downarrow} c_{\mathbf{k} \uparrow}+\Delta_{\mathbf{k}} c_{\mathbf{k} \uparrow}^{\dagger} c_{-\mathbf{k} \downarrow}^{\dagger}\right)$

where $\Delta_{\mathbf{k}} \simeq \hat{\Delta}\left(k_{x}-i k_{y}\right)^{2}$ at small $\mathbf{k}$. The structure of the solution is similar to the spinless case. However, the dispersion relation is now

$$
E_{\mathbf{k}}=\sqrt{\left(k^{2} / 2 m^{*}-\mu\right)^{2}+\left|\hat{\Delta}_{\mathbf{k}}\right|^{2} k^{4}}
$$

so at the transition point $(\mu=0), E_{\mathbf{k}}=k^{2}\left(|\hat{\Delta}|^{2}+\right.$ $\left.\left(2 m^{*}\right)^{-2}\right)^{1 / 2}$. For $\mu$ nonzero,

$$
E_{\mathbf{k}} \simeq|\mu|-\frac{k^{2}}{2 m^{*}} \operatorname{sgn} \mu,
$$

at small $\mathbf{k}$, which implies there is a minimum at nonzero $\mathbf{k}$ in the weak-pairing phase, $\mu>0$.

The position-space wavefunction for $N$ particles, of which $N / 2$ have spin $\uparrow, N / 2$ have $\downarrow$, has the form

$$
\Psi \propto \operatorname{det} g\left(\mathbf{r}_{i \uparrow}-\mathbf{r}_{j \downarrow}\right),
$$

where $g$ is the inverse Fourier transform of $g_{\mathbf{k}}=v_{\mathbf{k}} / u_{\mathbf{k}}$ (this is equivalent to a result in Ref. [2], p. 48). In the strong-pairing phase, $v_{\mathbf{k}} / u_{\mathbf{k}} \sim\left(k_{x}-i k_{y}\right)^{2}$, and $g(\mathbf{r})$ falls rapidly with $\mathbf{r}$. In the weak-pairing phase, $v_{\mathbf{k}} / u_{\mathbf{k}} \sim\left(k_{x}+\right.$ $\left.i k_{y}\right)^{-2}$, we find

$$
g(\mathbf{r}) \propto \bar{z} / z
$$

for large $\mathbf{r}$. Thus $|g| \sim$ constant, and $g$ is very long-range. At the critical point, $v_{\mathbf{k}} / u_{\mathbf{k}} \sim|\mathbf{k}|^{2} /\left(k_{x}+i k_{y}\right)^{2}$ (with a coefficient that depends on $\hat{\Delta}$ and $m^{*}$, unlike the p-wave case) and

$$
g(\mathbf{r}) \propto 1 / z^{2}
$$

This is the same behavior as in the Haldane-Rezayi (HR) state [13], when the latter is interpreted in terms of pairing of composite fermions [14]. Therefore we suggest that the HR state is precisely at the weak-strong pairing transition point, and has gapless excitations in the bulk.

Further evidence for the criticality of the HR state comes from the ground states on the torus. For the quasiparticle effective Hamiltonian $K_{\text {eff }}$, the presence of two spin states means that the $\mathbf{k}=0$ states, which occur only for ++ boundary conditions (see Section III), can be unoccupied in the strong-pairing phase, and doubly-occupied in the weak-pairing phase. Thus there is a total of four ground states, all with $N$ even, and none with $N$ odd, in 
both phases. However, at the critical point, $E_{\mathbf{k}=\mathbf{0}}=0$, and the $\mathbf{k}=\mathbf{0}$ state can be occupied zero, one, or two times, with no energy penalty. Hence for ++ boundary conditions, there are two ground states for $N$ even, and two for $N$ odd. The latter pair, in which $\mathbf{k}=\mathbf{0}$ is singly occupied, form a spin-1/2 doublet. Thus (on including the three ground states in the other sectors) there is a total of five ground states for $N$ even, two for $N$ odd. This is exactly what was found for the ground states of the hollow-core model of HR [13], (for which the HR state is the exact ground state on the sphere), both numerically [75,44 and analytically 44. The long distance behavior of the wavefunctions implied by the present approach agrees with that in Ref. 44, as in earlier cases. Also, we cite the energy spectrum of the hollow-core model, which was obtained numerically for $N=8$ particles on the sphere in Ref. 44. No clear gap can be seen in the spectrum. In view of these results, other numerical work on this model should also be reconsidered. Analytical results on zero-energy "edge" and "quasihole" states of the hollow core model [42,44], remain valid, but the earlier interpretation assumed a fully-gapped bulk ground state, and so if the bulk is gapless, the questions about the conformal field theory pictures of the bulk wavefunctions and the fermionic edge excitations [14, 41, 42, 45] are presumably moot. It is actually quite interesting that the hollow-core model (like the Ho plus three-body model in Section [II]) is critical. In this it resembles also certain other special Hamiltonians for which the exact ground states are known [76, 77]; the latter cases involve pairing of composite bosons.

\section{B. Structure of the weak-pairing abelian phase}

It is now of interest to find the properties of the dwave weak-pairing phase (the strong pairing phase has the same rather trivial properties as the others discussed previously). This phase has been discussed recently [57]. Here we wish to consider its application as a paired state in the FQHE. There are differences in the symmetry here compared with Ref. [57, which necessitate a certain amount of discussion. As we will see, the method of analysis of the edge and vortex states used previously does not seem satisfactory in the present case. Therefore, it seems necessary to use a more devious approach, which we now describe.

It is not difficult to see that, in the weak-pairing phase, the map from $\mathbf{k}$ to $u_{\mathbf{k}}, v_{\mathbf{k}}$ is topologically non-trivial and has $m=2$, that is, it wraps around the sphere twice. Because of this and its d-wave $(l=-2)$ symmetry, it has double zeroes at $\mathbf{k}=\mathbf{0}$ and $\mathbf{k}=\infty$. While such behavior at $\infty$ can be regarded as fixed by requirements of convergence and finite particle number, as mentioned earlier, that at $\mathbf{k}=\mathbf{0}$ is non-generic from the topological point of view. More generically, the map could pass over the south pole $\mathcal{S}$ in Anderson pseudospin space at two different $\mathbf{k}$ values, but this would require that the rotational symmetry be broken.

It will be useful to analyze such generic behavior in order to find the properties even of the d-wave case, as we will argue below. A convenient way to break rotational symmetry is to introduce an s-wave component $\Delta_{\mathbf{k} s}$ of the gap function $\Delta_{\mathbf{k}}$, in addition to the d-wave part $\Delta_{\mathbf{k} d}$ (s-wave is the simplest choice, and the gap function must remain even in $\mathbf{k}$, to retain the spin-singlet ground state). Then at small $\mathbf{k}$ the behavior is

$$
\Delta_{\mathbf{k}} \simeq \Delta_{s}+\hat{\Delta}\left(k_{x}-i k_{y}\right)^{2}
$$

and $\Delta_{s}$ and $\hat{\Delta}$ are both complex. In this case, when $\Delta_{\mathbf{k} s}$ is a sufficiently small perturbation on the weak-pairing d-wave phase, the effect is that $E_{\mathbf{k}}$ has a minimum at just two nonzero values of $\mathbf{k}$, of the form $\pm \mathbf{k}_{0}$ by symmetry. This does not change the topology of the $u_{\mathbf{k}}$, $v_{\mathbf{k}}$, and since the system is fully gapped as far as the quasiparticles are concerned, it should not change any physical properties. However, if $\Delta_{\mathbf{k} s}$ is large and dominates $\Delta_{\mathbf{k} d}$ for all $\mathbf{k}$, then the system is essentially in an s-wave state which must be topologically trivial. This is the same phase as the strong-pairing d-wave phase, even though the rotational symmetry is different; these limits can be connected as $\Delta_{\mathbf{k} s}, \Delta_{\mathbf{k} d}$, and $\mu$ are varied, without crossing another transition. Therefore there must be a phase transition as $\left|\Delta_{\mathbf{k} s} / \Delta_{\mathbf{k} d}\right|$ varies at fixed $\mu>0$, similar to those discussed above. In this case, the dispersion relation $E_{\mathbf{k}}$ vanishes linearly at two points $\pm \mathbf{k}_{0}$ at the transition, and the map from $\mathbf{k}$ to $u_{\mathbf{k}}, v_{\mathbf{k}}$ is discontinuous at these two points. These will be points where $\xi_{\mathbf{k}}=k^{2} / 2 m^{*}-\mu=0$, as well as $\Delta_{\mathbf{k}}=0$, and so occur at some $\mu>0$. As $\mu \rightarrow 0, \mathbf{k}_{0} \rightarrow 0$, and these points coalesce to give the previous discussion in which $\Delta_{\mathbf{k} s}=0$ for all k.

The two points $\pm \mathbf{k}_{0}$ at which $E_{\mathbf{k}}$ has a conical form give a spectrum similar to a spin-1/2 doublet of Dirac fermions. This is similar to behavior well-known in other condensed-matter models, including fermions on a lattice in a magnetic field, and d-wave pairing with a $\mathrm{d}_{x^{2}-y^{2}}$ structure (perhaps induced by a square lattice) [57. By concentrating on the degrees of freedom near these points, and shifting them in $\mathbf{k}$-space to the origin (which produces oscillating factors in real space correlation functions of the fermions), the fermion excitations can be mapped onto a complex (i.e. Dirac, not Majorana) spin-1/2 doublet of relativistic fermions for each pair of such points in $\mathbf{k}$ space. Near the transition point, or by varying parameters in the other models mentioned (the $\mathrm{d}_{x y}$ part of the gap function in the second model), the Dirac fermions gain a mass. If we now apply to this a similar analysis for edges to that we used previously when the minima were at $\mathbf{k}=0$, then we find that the edge excitations form a spin- $1 / 2$ doublet of chiral Dirac 
(or Weyl) fermion fields [57], and we can give a similar analysis for the vortices.

For our purposes, we are interested in unbroken rotational symmetry in the bulk, so the preceding remarks do not seem to apply directly. However, if we analyze the edge excitations using the method of previous sections applied to the d-wave model, by simply considering the effect of $\mu$ changing sign on a domain wall, we do not find any edge modes. But we are suspicious of this result because of the nongeneric form of $u_{\mathbf{k}}, v_{\mathbf{k}}$, and previously we were relying on the assumption that the results are robust because of the topological nature of the phenomena. In the present case, the results should be the same as if we examine a domain wall caused by varying $\Delta_{\mathbf{k} s}$ so as to cross the transition, because the phases are the same, and therefore the edge state and vortex properties should be those of the Dirac fermion doublet. We expect that what happens, even if the bulk phases are rotationally invariant, is that the edge or domain wall breaks rotational symmetry, and induces a splitting of the zeroes of $\Delta_{\mathbf{k}}$, in so far as this function in $\mathbf{k}$ space is meaningful. With the symmetry broken, the previous analysis can be applied. Of course, what should be done in all cases is a full solution of the BdG equations with the reduced symmetry, and of the gap equation self-consistently. This is clearly difficult, though it has sometimes been attempted, and a deeper analysis that explains why arguments of the type we have given yield the correct results would be preferable. We will attempt to give such an argument below. We note that, if we consider perturbations analogous to the s-wave component in the cases in earlier sections, we do not find any change in those results.

A feature of the Dirac-like nature of the fermion edge spectrum is that there are two doublets of fermion modes, particles and antiparticles. These correspond to the two points $\pm \mathbf{k}_{0}$ in $\mathbf{k}$ space from which they arose. This can be described by saying that there is a $\mathrm{U}(1)$ quantum number or "charge", which we will call $M$, and that the particle and antiparticle carry opposite values of $M$. It may be that the additional $\mathrm{U}(1)$ symmetry, which must not be confused either with any part of the $\mathrm{SU}(2)$ of spin, or with the $\mathrm{U}(1)$ of underlying particle-number conservation, which is already broken spontaneously by the pairing, is in fact broken by the dynamics, since there seems to be no symmetry to protect it. However, as in the case of the 331 state plus tunneling, even when the symmetry is broken, the counting of edge excitations, and the statistics properties, etc, should be unchanged.

If we continue to assume the $\mathrm{U}(1)$ symmetry exists, for the sake of the latter analysis, then the doublet of chiral Dirac fermions on an edge in fact has a larger symmetry (note that there are no interactions to consider in the theory). The fields are equivalent to four Majorana fermions, and there is $\mathrm{SO}(4)$ symmetry. We note that, as Lie algebras, $\mathrm{SO}(4) \cong \mathrm{SU}(2) \times \mathrm{SU}(2)$, and here the first $\mathrm{SU}(2)$ can be identified with the spin-rotation symme- try group, while the second contains the $\mathrm{U}(1)$ symmetry just discussed as a subalgebra, generated by, say, rotation about the $z$ axis in the second space. Thus the Dirac field and its conjugate can be viewed as carrying spin $M= \pm 1 / 2$ under the second $\mathrm{SU}(2)$. Alternatively, viewing the theory just as two Dirac fields, these can be bosonized, and we obtain two chiral bosons. The allowed "charge" states for the edge, which take values in the Cartan subalgebra of $\mathrm{SO}(4)$, lie on a two-dimensional lattice. This lattice is a direct product of two copies of the weight lattice of $\mathrm{SU}(2)$. Points in the lattice simply describe the total $S_{z}$ of spin and the total $M$ on the edge. The same desciption applies to a vortex, since we can view it as an edge rolled up into a small circle. In the latter case, the half-flux quantum we assume in the vortex corresponds to changing to periodic boundary conditions for the fermions on the straight edge, for all components of the fermions. In addition to these different boundary condition sectors, there are also selection rules from the global quantum numbers. These are similar to the rules described in detail in 42. Specifically, for $N$ even, one can have the ground state with no edge excitations, or one can create fermions on the edge, but only in even numbers. In the present situation, each of these fermions can be in any of the four states in the representation of spins $(1 / 2,1 / 2)$ under $\mathrm{SU}(2) \times \mathrm{SU}(2)$. For odd total fermion number, there must be one unpaired fermion, which we can put on the edge to obtain a low-energy state. Then the charge (or particle number) differs by one from the ground state, in addition to the non-trivial $\mathrm{SO}(4)$ quantum numbers. Additional fermion pairs can be excited in this case also. If we consider two parallel edges, as for a system on a cylinder, then we build up the full spectrum by applying these rules to the two edges together, and there will be different sectors corresponding to the presence of either zero or one-half of a flux quantum threaded through the cylinder. In the FQHE application, there are also chiral bosons for charge excitations on the edge, and there are fractional charge sectors for each edge, though the total charge must be integral, and the total charge (or particle number) is correlated with the fermion excitations through its parity, as already explained.

The quantum numbers of the vortices (or FQHE quasiparticles) correspond closely to the possible quantum numbers for the edges, and are obtained in a similar way. As a simple example, we again consider two vortices. The situation is equivalent to the existence of a single fermion zero mode for the pair, which can be occupied by any of four types of fermions. Thus there is a total of 16 states, of which half have $N$ even, half have $N$ odd. These should be analyzed as a product of four states possible for each vortex. The states for a vortex transform as $(1 / 2,0) \oplus(0,1 / 2)$ under $\mathrm{SO}(4)$, that is they either carry spin $1 / 2$ and no $M$, or vice versa. These correspond to the states for two edges with a half flux 
quantum through the cylinder, in which the zero mode shared by the two edges can be occupied by any of the four types of fermions. For any even number of vortices, the results are similar, and the counting of degenerate low-energy states is fully accounted for by the four states for each vortex, and hence there is no nonabelian statistics. Thus a d-wave paired state, or even a superconductor, in two dimensions, possesses vortices that may carry spin $1 / 2$, but not simply because a fermionic quasiparticle can sit on the vortex. In the FQHE, these vortices also have well-defined charge of $\pm 1 /(2 q)$ for filling factor $\nu=1 / q$, where $q$ must be even when the particles are fermions (such as electrons). This is also true in all other cases discussed in this paper. It arises from the effective half quantum of flux that the vortices carry, and they can exist only in even numbers if the system has no edges.

We hope that the above discussion gives a sufficient impression of the quantum numbers of the vortex states. For readers familiar with the general theory of abelian FQHE states, we will now give a precise definition of the structure of the state, using the Gram (or K) matrix language, which specifies the lattice formed by the possible quantum numbers (including charge) of the FQHE quasiparticles, as well as their statistics, and the order parameters and chiral algebra of the edge theory [48, 49, 78, 42].

The full lattice of possible quantum numbers of a vortex, or the total quantum numbers of a set of multiple vortices, is denoted $\Lambda^{*}$ (as in Ref. [48]). We will describe it first as a set of vectors in $\mathbf{R}^{3}$, using an orthonormal basis with the standard inner product. Then $\Lambda^{*}$ consists of the set of vectors of the form

$$
\mathbf{v}=\left(r_{1} / \sqrt{2}, r_{2} / \sqrt{2}, r_{3} /[2 \sqrt{q}]\right)
$$

where $r_{1}, r_{2}, r_{3}$ are integers obeying $r_{1}+r_{2}+r_{3}=0$ $(\bmod 2)$. Also the statistics of the excitation is $\theta / \pi=$ $\mathbf{v}^{2}$, where $\theta$ is the phase picked up by exchanging two identical such quasiparticles, and the conformal weight of the corresponding operator in the edge theory is $\mathbf{v}^{2} / 2$. The basis has been chosen so that the three quantum numbers carried by the excitations are proportional to the components in this basis. In fact, the spin $S_{z}=r_{1} / 2$, $M=r_{2} / 2$, and the charge is $Q=r_{3} /(2 q)$. From these rules we see that the smallest possible vortex (the one with the smallest nonzero $\mathbf{v}^{2}$ ) carries either $S_{z}= \pm 1 / 2$ or $M= \pm 1 / 2$, and charge $\pm 1 /(2 q)$, as stated above, and has statistics $\theta / \pi=1 / 2+1 /(4 q)(\bmod 2)$. It is easy to verify that the full lattice $\Lambda^{*}$ is obtained as the set of all linear combinations, with integer-valued coefficients, of the vectors describing the quantum numbers of the basic (smallest) vortices, and thus represents the possible quantum numbers of any collection of vortices.

The excitation lattice $\Lambda^{*}$ is the dual lattice to the condensate lattice $\Lambda$, and $\Lambda$ is a sublattice of $\Lambda^{*}$. In the present case, $\Lambda$ is the set of vectors $\mathbf{w}$ that are linear combinations with integer coefficients of the vectors
$( \pm 1 / \sqrt{2}, \pm 1 / \sqrt{2}, \pm \sqrt{q})$ (with all three signs independent) which represent the underlying particles. These represent possible states for a particle (electron) tunneling into an edge, and these electron operators, as we may term them in spite of the emergent $M$ quantum number, are usually part of the condensate lattice, as they are in the hierarchy theory. (An exception to this is the strong-pairing phases, where only operators of charge a multiple of two appear.) The fact that $\Lambda$ and $\Lambda^{*}$ are dual means that for any $\mathbf{v} \in \Lambda^{*}$, we have $\mathbf{v} \cdot \mathbf{w} \in \mathbf{Z}$ for all $\mathbf{w} \in \Lambda$, and vice versa. It suffices to check this for the w's representing the electron operators.

Both lattices possess neutral sublattices, that is lattices of vectors such that $Q=0$. The neutral sublattice of $\Lambda$, denoted $\Lambda^{\perp}$, consists of vectors with $r_{1}$ and $r_{2}$ even. Thus these form a direct sum $\mathbf{Z} \oplus \mathbf{Z}$ of onedimensional lattices. Each of the latter can be viewed as the root lattice of $\mathrm{SU}(2)$ in Lie algebra theory, and $\Lambda^{\perp}$ is the root lattice of $\mathrm{SO}(4)$. The neutral sublattice of $\Lambda^{*}$, denoted $\Lambda^{* \perp}$, is the set of vectors with $r_{3}=0$, and so $r_{1}+r_{2}=0(\bmod 2)$. This is a sublattice of the weight lattice of $\mathrm{SO}(4)$, which would be the dual of $\Lambda^{\perp}$ as a two-dimensional lattice. The simplest nontrivial neutral vector is of the form $( \pm 1 / \sqrt{2}, \pm 1 / \sqrt{2}, 0)$ (with independent plus and minus signs), and these represent the neutral fermions, that is the BCS quasiparticles considered in this paper. These cannot be created individually on a single edge; only excitations lying in $\Lambda$, such as even numbers of such fermions, can be 42.

An integral basis for a lattice is a set of vectors in the lattice that are linearly independent (over $\mathbf{R}$ ), such that all vectors in the lattice can be written as linear combinations of those in the set, with integer coeffecients. Such a basis cannot be an orthogonal set of vectors, unless the lattice is a direct sum of one-dimensional lattices. In our case, a convenient integral basis for $\Lambda$ (other than a suitable set of three of the electron operators above), is $\mathbf{e}_{1}=(1 / \sqrt{2}, 1 / \sqrt{2}, \sqrt{q}), \mathbf{e}_{2}=(\sqrt{2}, 0,0), \mathbf{e}_{3}=(0, \sqrt{2}, 0)$. The Gram matrix of the lattice is the set of inner products of these vectors, $G_{i j}=\mathbf{e}_{i} \cdot \mathbf{e}_{j}$, and in this case is

$$
G=\left(\begin{array}{ccc}
q+1 & 1 & 1 \\
1 & 2 & 0 \\
1 & 0 & 2
\end{array}\right)
$$

The diagonal structure of the lower-right $2 \times 2$ block reflects the direct product nature of the $\mathrm{SO}(4)$ root lattice. The determinant of $G$, $\operatorname{det} G=4 q$, determines the index of $\Lambda$ as a subgroup of $\Lambda^{*}$, that is the number of equivalence classes of vectors in $\Lambda^{*}$ modulo $\Lambda$. It gives the number of ground states of the system on a torus, or the number of sectors of edge states. Factoring off the center of mass degeneracy $q$, we find that there are four, in agreement with the analysis based on the quasiparticle effective Hamiltonian. The Gram matrix, together with distinguished vectors that specify the charge $Q$ and 
spin $S_{z}$ quantum numbers, is sufficient information from which to reconstruct the lattices $\Lambda$ and $\Lambda^{*}$, and hence the universal aspects of the phase, such as ground state degeneracies, quasiparticle statistics, and the theory of the edge states. In this context, the Gram matrix is often called the $\mathrm{K}$ matrix.

This completes the analysis we will give of the phase. It is a generalized hierarchy state in the sense of Ref. [48], and resembles the 331 phase. The latter lacks the $\mathrm{SU}(2)$ of spin and hence has a two-dimensional lattice; its structure was described in detail in Ref. 42,44.

We may now compare the universal properties of this state with others analyzed previously. We find that several other constructions of this spin-singlet phase have already been given. In Ref. [48], this was mentioned briefly as the structure of both a spin-singlet state for $\nu=1 / 2$ proposed by Jain [22 (see also Ref. 72]), and one proposed by Lee and Kane 74. A more detailed analysis was given in Ref. [73], where it was also identified with a hierarchical construction. In the latter, one starts with the Halperin spin-singlet 2/5 state [1], which is a 332 state in the Coulomb plasma language, and then one makes a finite density of spinless quasielectron excitations of that state, each carrying charge $2 / 5$. The quasielectrons are then put in a Laughlin $1 / 2$ state, to obtain a singlet state with $\nu=1 / 2$. The hierarchical step implies that the resulting state has a three-dimensional lattice. In Ref. [73, this and the Jain construction were shown to coincide. Unfortunately, the formulas there contain a small mistake: the final basis vector in Eq. (4.8) in that reference should be reduced by a factor of two, as should the entries in the first row and column of the Gram matrix in Eq. (4.9) there. The resulting matrix is then identical to $G$ above, with $q=2$, after permuting the basis vectors. This basis is the natural one for the hierarchy approach. In our $G$ above, the top left $2 \times 2$ block (with $q=2$ ) describes the Halperin 2/5 state, and reflects its origin. (The $2 / 5$ state itself has the same $G$ as the spin-polarized hierarchy $2 / 5$ state, which descends from $\nu=1 / 3$, as reflected by the $q+1=3$ at the top left.)

The Jain $\nu=1 / q$ spin-singlet state was proposed as a trial wavefunction, namely

$$
\Psi=\chi_{1}^{q-1} \chi_{2} \chi_{1,1} .
$$

Here $\chi_{m}$ stands for the wavefunction for $m$ filled Landau levels of spinless particles, (so $\chi_{1}$ is the Vandermonde determinant or Laughlin-Jastrow factor) and $\chi_{1,1}$ is the lowest Landau level filled with particles of both spins. We ignore the Gaussian factors in these wavefunctions, and have omitted the projection to the LLL. The filling factor is again $1 / q$. This wavefunction can be loosely viewed as a Coulomb plasma of particles carrying charge, spin and another quantum number $M= \pm 1 / 2$ to represent the two Landau levels in $\chi_{2}$. The exponents in the wavefunction correspond to inner products of corresponding vectors, which are just those of the four electron operators with $Q=1$. This establishes the equivalence, as for the hierarchy states in Ref. [48]. In fact, the extra $\mathrm{SU}(2)$ that appears here acts on the LL indices in $\chi_{2}$ in Jain's function, just like the $\mathrm{SU}(n)$ that appeared for $n$ Landau levels in the spin-polarized Jain states [22] and the corresponding hierarchy states with $n$ levels in the hierarchy. The state is a combination of the Halperin spin-singlet structure with the spinless composite-fermion/hierarchy structure. We should point out that the number of flux for Jain's state on the sphere is $N_{\phi}=q(N-1)-2$, the same as for the HR state, which follows from our analysis, and was noticed previously [72]. This is of course essential in order for it to be possible to vary parameters smoothly to reach the transition.

Thus the same $\nu=1 / 2$ (more generally, $\nu=1 / q$ ) spin-singlet phase has arisen in four different ways. We want to emphasize that the equivalence of the universal long-distance properties does not mean that the trial wavefunctions in different approaches are the same. For example the paired wavefunction found here and Jain's above do not look alike. It may be that one is a much better description (has a much larger overlap with an exact ground state) for medium size systems than the other, even though they describe the same phase. The equivalence found here is analogous to that between the 331 state and the A-phase p-wave state, however in that case there was an exact equivalence of certain wavefunctions through the Cauchy determinant identity. We may still expect some equivalence in the long-distance form of the wavefunctions.

\section{Induced Chern-Simons actions and analogs of the Hall conductivity}

The arguments given so far for the edge states and for zero modes on vortices, on which the identifications of the weak-pairing phases have been based, may appear not to be very well-founded, as they have been based on analyzing the BdG equations for special forms of the gap function and variation of the parameter $\mu$, though we did argue by continuity that the states found must persist as the equations are varied while staying in the same phase. In this Subsection, we argue that the results we have obtained are in fact very robust, because the edge states, and the form of the bulk theory described by the Gram matrix of the condensate lattice, are required as a consequence of transport properties of the bulk weak-pairing phases. These transport properties are the quantization of the spin and heat analogs of the Hall conductivity, which we prove explicitly for the spin case. They imply the existence of certain edge state structures, just as in the case of charge transport in the QHE, and when the weak-pairing phases correspond to abelian FQHE states, the Hall spin conductivity is actually a part of the Gram 
matrix description. The remainder of this Subsection discusses these points, but the technical details are relegated to the Appendix. We should point out that the form of the argument has already appeared in Ref. 57 for the cases with $\mathrm{SU}(2)$ or $\mathrm{U}(1)$ spin symmetries, though the explicit derivation of the Hall conductivities was not given there.

For the cases of spin-singlet pairing, and of p-wave pairing with an unbroken $\mathrm{U}(1)$ symmetry, we derive in the Appendix the Hall spin conductivity $\sigma_{x y}^{s}$, and show that in any fully-gapped translationally-invariant superconducting phase it is given by a topological invariant, which within the BCS approximation is proportional to the same winding number we discussed earlier in Sec. II and subsequently. Some similar statements appeared earlier, but were for the charge Hall conductivity «,, 8 , and also for the existence of a Hopf term and of a nonabelian CS term, in the two-dimensional A-phase with $\mathrm{SU}(2)$ symmetry broken spontaneously [5]. In this paper we restrict ourselves to the Hall conductivies for conserved quantities for which the corresponding symmetry is not broken spontaneously in the paired state, such as spin and energy. For quantities for which this is not true, such as charge, we do not find quantized Hall conductivities in superconductors (though of course we do find quantized Hall conductivity in the QHE systems). Since our point of view differs somewhat from that in, for example, Ref. [5], we give a self-contained discussion.

To be precise, for the Hall spin conductivity in a spinsinglet paired state, we find that if the particles are viewed as carrying spin $1 / 2$ (we usually set $\hbar=1$ ), the Hall response to an applied spin analog of the electric field, such as a gradient in the Zeeman splitting, is

$$
\sigma_{x y}^{s}=m \frac{(\hbar / 2)^{2}}{2 \pi \hbar}
$$

where $m$ is the winding number, which is \pm 2 in a d-wave weak-pairing phase. We have written the Hall spin conductivity in this form to emphasize the similarity to the usual $e^{2} / h$, with $e$ replaced by $\hbar / 2$ here. In $\hbar=1$ units, we obtain in our d-wave weak-pairing phase $\sigma_{x y}^{s}=1 / 4 \pi$. We chose the + sign, since in the FQHE applications of the $l=-2$ state, the edge modes propagate in the same direction as the charge modes. This agrees with Refs. [56,57, where different arguments were used. For the pwave case, we view the fermions as carrying isospin \pm 1 , and hence the Hall spin conductivity we obtain is

$$
\sigma_{x y}^{s}=m \frac{1}{2 \pi \hbar}
$$

where the winding number $m$ is \pm 1 in the weak-pairing p-wave phases, again with $m=1$ for the $l=-1$ case.

These results agree with the descriptions we have already given of the weak-pairing FQHE phases using the Gram matrix or lattice theory. In fact we should point out that a quantized Hall spin conductivity is not unusual in FQHE systems, though it is not always emphasized. It occurs for example in any spin polarized state, such as the integer and Laughlin states with $\nu=1 / q$, because the electrons carry spin $1 / 2$ as well as charge, and so the two Hall conductivities are proportional. It also occurs in some spin-singlet Hall states (abelian examples were discussed in [73]), including the $\nu=2$ state with the LLL filled with both spins, and the Halperin $m m n$ states with $m=n+1, m$ odd, which are a generalization of the $\nu=2$ state. In these cases, we obtain the full $\mathrm{SU}(2)$ version of the Hall spin conductivity, with $\sigma_{x y}^{s}$ taking the same value as in the d-wave weak-pairing phase. The same quantized Hall spin conductivity was also found in certain spin-liquid states for lattice antiferromagnets $[79]$. The wavefunctions of these states are the same as that of the Halperin state, with the charge degree of freedom removed (i.e. the wavefunction is $1 / 2,1 / 2,-1 / 2$ ), and the spin- $1 / 2$ particles restricted to lattice sites, explaining this result.

The result that the Hall conductivity, in units of (quantum number $)^{2} / h$, is a topological invariant given by an integral over $\mathbf{k}$ space, is similar to one form of the ordinary charge Hall conductivity, found originally for a noninteracting periodic system with a rational number of flux quanta per unit cell, as an integral over the Brillouin zone 80. In systems where $\mathbf{k}$ is not a good quantum number, such as the same system with irrational flux, or when disorder is present, or in other geometries, including those with edges, that break translational invariance, such a topological invariant is apparently not available. Yet the idea of quantization as resulting from the conductivity being a topological invariant that measures an intrinsic local property of the ground state seems too good to give up. For the noninteracting QHE with disorder, the topological invariant has been extended using noncommutative geometry, so that the Hall conductivity is an integral over a "noncommutative Brillouin zone", and in this way quantization has been proved even for the physically relevant case of a nonzero density of localized states at the Fermi energy 81. It would be interesting to extend this to other cases, including the paired states with disorder, which we discuss in the next Section. In the Appendix, we give arguments that the form we obtain is exact to all orders in the interactions, but only for a translationally-invariant system.

Now we can use the results on the Hall spin conductivity to argue that the edge state properties we have obtained are indeed correct. In the Appendix, we derive the Hall spin conductivities by obtaining the induced action for an external gauge field that couples to the spin or isospin. The actions that result (cf. Ref. 82]) are CS terms for an $\mathrm{SU}(2)$ gauge field in the spin-singlet (dwave) case, and for a U(1) gauge field in the p-wave case. Now, using either the Hall conductivities and arguing as in the QHE literature 83] (see also 84, 85,), or using the 
induced actions and arguing as in the field theory literature [86] (quite similar arguments appear in Ref. 87] and references therein), we can conclude that on a domain wall between phases with different $\sigma_{x y}^{s}$ 's (one of the phases might be the vacuum outside an edge, with $\left.\sigma_{x y}^{s}=0\right)$ there must be chiral edge excitations. In the presence of a uniform spin-electric field, a spin-current is induced in a region with $\sigma_{x y}^{s} \neq 0$, and the normal component of this at a domain wall has a discontinuity, representing a net inflow of spin onto the wall. To avoid violating the continuity equation for the spin density and spin current density, there must be chiral modes on the wall, and a "gauge anomaly" in the conservation of spin on the wall alone. The tangential field induces a nonzero divergence (i.e., an anomaly) of spin current along the wall, which cancels the net inflow from the bulk. Such an anomaly can occur only if gapless chiral excitations exist on the domain wall. The minimal chiral theory required to produce the anomaly is the usual chiral Luttinger liquid (or chiral Gaussian model) in the $\mathrm{U}(1)$ case, with the value of the coupling that corresponds to a free chiral Dirac (Weyl) fermion in $1+1$ dimensions, and the $\mathrm{SU}(2)$ chiral Wess-Zumino-Witten (WZW) theory 88] with $k=m / 2=1$ in the $\mathrm{SU}(2)$ case. All of this applies even within the BCS mean field framework we used before, and then the edge excitations must be free fermions. In the $\mathrm{p}$-wave case, we therefore expect simply a single chiral Dirac fermion to propagate on the edge. In the d-wave case we must have an $\mathrm{SU}(2)$ doublet of chiral Dirac fermions, which can be represented by the $k=1$ chiral WZW model, together with an additional chiral $\mathrm{U}(1)$ degree of freedom, which we argued earlier must exist, and called $M$. We have therefore reproduced the claimed results about the edge states, neglecting the charge degree of freedom.

We note that, when formulating such arguments for the nature of the chiral edge theories, we can presumably assume that the theories are unitary, conformal fields with local current operators for physical conserved quantities (as usual, Lorentz invariance may be spoiled by the presence of different velocities for different excitations, but this will not matter for the statistics and other universal properties in which we are interested). Previously, we might not have assumed this, because of the example of the Haldane-Rezayi (HR) state in particular. But we have learned that the HR state is at a critical point, and previous discussions of the edge and quasiparticle properties of that state are irrelevant. Thus, with the demise of the HR state as a bulk phase, it becomes attractive to believe that the edge theories of incompressible FQHE phases are always unitary conformal field theories. With this assumption, in theories with $\mathrm{SU}(2)$ Hall spin conductivities, unitarity of the edge theory requires quite generally that $k$ be an integer [88,39], and so $\sigma_{x y}^{s}=k /(4 \pi)$ in the above conventions.

The preceding arguments do not apply to the spinless p-wave case, in which there is no continuous unbroken symmetry. This is unfortunate in view of the great interest in the nonabelian properties of the weak-pairing phase. But there is another Hall-type conductivity, which exists in all cases, including those without a continuous symmetry. This is the Leduc-Righi (LR) conductivity, which is the $x y$ component $\kappa_{x y}$ of the thermal conductivity, and is of course related to the transport of energy, a conserved quantity. Like the Hall conductivities for charge and spin, this is a non-dissipative transport quantity that can only be nonzero when parity and timereversal symmetries are broken. In systems with a gap for all bulk excitations and with chiral edge excitations, it can be argued that the LR conductivity is nonzero 89, 57. Thus this applies to QHE systems, and to superconductors (paired systems) if there is no gapless collective charge mode. The value of $\kappa_{x y}$ can be obtained [39.57 by considering a sample with two edges and a small temperature difference between the edges (strictly speaking, the following argument yields the LR conductance, not the conductivity). The chiral excitations on each edge are excited by different temperatures, and this produces a larger heat current on one edge in one direction than that on the other edge in the other, and hence a net heat current. This shows that the current is related to the heat capacity of each mode on an edge, times the velocity of the mode, summed over modes. It is known that the heat capacity for each mode is related to the Virasoro central charge $c$ in the conformal field theory of the edge excitations [90]. In $\kappa_{x y}$, the velocities cancel, and the remaining number is proportional to the total central charge of the edge theory (including the charge modes in the FQHE); precisely,

$$
\kappa_{x y}=c \frac{\pi^{2} k_{B}^{2} T}{6 \pi \hbar}
$$

This is the fundamental relationship governing the $L R$ conductivity in all quantum Hall problems. Here we assumed that all modes on an edge propagate in the same direction; if not, then $c$ should be replaced by the difference of the central charges for the right and left-moving theories. Related to this, the two-probe thermal conductance of such a system is also equal to $\kappa_{x y}$ (for a case with all edge modes propagating in the same direction), just as the two-probe conductance is equal to the quantized $\sigma_{x y}$ of the bulk. It has been found that the two-probe thermal conductances of the Laughlin states at various $\nu=1 / q$ are independent of $q$, even though the theories of the chiral Luttinger liquids contain $q$ as a parameter [91. This is because the central charge is $c=1$, independent of $q$.

The formula for $\kappa_{x y}$ is similar in structure to that for the Hall spin conductivity. However, the central charge $c$ does not have to be an integer, and indeed for a single Majorana fermion, $c=1 / 2$ [92], so that for free fermions in general, $c$ is a multiple of $1 / 2$. Nonetheless, we do 
expect it to be quantized, in the sense of invariant under small deformations of the theory (including adding weak disorder).

The argument above for the LR conductivity made use of the edge states. However, we want to use it to back up our results on the fermionic edge modes of the MR state, in analogy with the arguments for the other cases, which used the Hall spin conductivities. Hence we need an independent argument for the value of the LR conductivity. We believe that it should be possible to derive such a result, analogously to the Hall spin conductivities, by considering the system in external gravitational fields. Here the Christoffel symbols, or the spin connection, play the role of the external gauge fields we used in the spin case, but should be viewed as determined by the metric of spacetime, which we treat as the independent variable and set to the usual Minkowski metric after calculating responses. The role of the currents, to which the vector potentials couple, is played by the energy-momentum tensor, which includes the energy flux among its components. The significance of changing the metric should be clear if we recall that equilibrium systems can be represented in imaginary time, with the imaginary time direction periodic, the period being $1 / k_{B} T$. Thus a temperature gradient might be viewed as changing the geometry of space and (imaginary) time. A more rigorous derivation would avoid imaginary time, but should still involve the response to changes in the metric. The leading term in the induced action for the external gravitational field in $2+1$ dimensions will in general be the gravitational CS term, which can be written in terms of the spin connection in close analogy to the usual CS terms:

$$
\frac{1}{4 \pi} \frac{c}{24} \int d^{3} r \epsilon_{\mu \nu \lambda} \operatorname{tr}\left(\omega_{\mu} \partial_{\nu} \omega_{\lambda}+\frac{2}{3} \omega_{\mu} \omega_{\nu} \omega_{\nu}\right)
$$

where we view the spin connection $\omega$ as a $3 \times 3$ matrix, determined by the metric. (Such a term was also proposed earlier for $\mathrm{He}^{3}$-A films in Ref. [7].) The coefficient contains $c / 24$, which shows the relation to the central charge $c$ in a corresponding chiral conformal field theory on a boundary [39], is needed to cancel the anomaly in energy-momentum conservation on the boundary by an inflow (LR "Hall" current) from the bulk [86, as for the charge and spin Hall conductivities.

We have not completed a calculation (analogous to those in the Appendix) of the induced action or LR conductivity for the bulk from first principles. It would involve coupling the underlying system to an arbitrary metric, then using Ward identities to relate the response to a topological invariant. However, if we assume a spectrum of relativistic fermions at low energies, with a minimal coupling to the gravitational field, then the calculation can be carried out, and is known in the literature [93]. The coefficient of the induced gravitational CS term again has the form of the same topological invariant as in the Appendix, but integrated over only half the sphere, as in the similar treatment of the spin cases. For a single Majorana, this yields the above form with $c$ replaced by $\pm 1 / 4$ on the two sides of the transition. Inclusion of the contribution of a Pauli-Villars regulator then produces $c=0$ on one side, $c=1 / 2$ on the other. We expect that for our nonrelativistic system, where the large $\mathbf{k}$ behavior is explicitly known, we would obtain such a result also, with $c$ in a general system of paired fermions being proportional to the same topological invariant (winding number) we have seen already, and (allowing for factors of two associated with spin degeneracy) we would find $c=1 / 2$ in the spinless $\mathrm{p}$-wave weak-pairing phase, and also $c=1$ (2) in the triplet p-wave (singlet d-wave) cases. (These are the results for the paired fermions, and in the FQHE would have to be supplemented by adding $c=1$ for the charge degrees of freedom.) Therefore we believe that the existence of the edge states and vortex degeneracies in the MR phase can be placed on a firm footing.

A somewhat related issue is to obtain effective actions describing the weak-pairing phases. We emphasize that the CS actions discussed in this Subsection are induced actions for external fields which act as sources, and should not be confused with effective actions, which contain fields that should be functionally-integrated over, and represent the dynamics of the system at low energies and long wavelengths. For the abelian FQHE states, the effective actions fall into the framework of the known theory, based on the Gram (K) matrix [78. For the MR, and other similar, phases, something different is required. For the abelian phases, there is an evident similarity between the induced and effective actions. For example, in the d-wave case, neglecting the charge degree of freedom, one would expect the bulk effective action to be an $\mathrm{SU}(2) \times \mathrm{U}(1)$ CS theory, with $k=1$ for the $\mathrm{SU}(2)$ part (here, in the effective action, $k$ must be an integer to maintain gauge invariance). This theory is determined by the requirement that it produce the correct edge theory 39. (The U(1) part could possibly be extended to a second $\mathrm{SU}(2)$ with $k=1$, to agree with the $\mathrm{SO}(4)$ edge theory discussed above.) It is known that such an effective theory also produces the desired induced gravitational CS term in all cases [39]. By analogy, we are led to expect that the MR phase, where the edge theory involves only the $c=1 / 2$ representations of the Virasoro algebra, can be described by an effective theory which is some sort of gravitational CS theory (similarly, there were earlier proposals for gravitational-type effective actions for three-dimensional paired states [6]). Quantization of such a theory should yield Virasoro conformal blocks in the same way that quantization of CS theory yielded current algebra blocks [39], and thus be closely related to the wavefunctions discussed in Sec. II and in [14,44. This hope is encouraged by the identification of the parameter $\hat{\Delta}$ and the vector potential $A$, which should be functionally integrated over in the full treatment, as the vielbein and spin connection of $2+1$ gravity, 
or at least as the part relating to $\mathrm{SO}(2)$ rotations of space only, as discussed in Sec. II. The hope of producing conformal blocks in this manner from a gravitational analog of CS theory has been around for a long time 39, but does not seem to have reached fruition, in spite of an interesting attempt by Verlinde 94]. Such a theory would be an interesting, possibly more natural, alternative to the "conventional" approach, along lines anticipated in Ref. [14], of a CS analog of a coset construction [47.

We also wish to comment on whether our results imply that fractional and nonabelian statistics occur in paired superfluids, as opposed to FQHE systems. For example, the weak-pairing $\mathrm{d}$-wave and spin-triplet $\mathrm{p}$-wave phases have nontrivial Hall spin conductivity, and the smallest possible vortices carry spin (or $M$ ) quantum numbers. Hence the spin degrees of freedom contribute a fractional amount to the Berry phase on exchanging such vortices. Similarly, exchanging vortices in the weak-pairing spinless p-wave phase should produce a matrix action on the space of degenerate states we have identified, which we may be tempted to term nonabelian statistics. However, although these contributions from the spin (or fermion number) sector are well-defined, in a neutral superfluid the charge degree of freedom is gapless, and the vortices act on the charge (particle number) variables also, so as with vortices in a simple neutral superfluid, which carry no well-defined particle number, the contribution to the total Berry phase is not well-defined, due to the charge sector [95]. Nonabelian statistics is still meaningful, modulo phase factors. In the incompressible FQHE phases, this problem disappears, and the statistics properties have been characterized above, in detail for the abelian cases. Also, in a superfluid with a Coulomb interaction, there is again no problem, even if the interaction is $\sim 1 / r$, which does not produce a plasmon gap in two dimensions. Vortices are neutral because of screening, and so nonabelian statistics, or fractional statistics in the triplet p-wave case, can occur, with no contribution from the charge sector. There are also "neutral" vortex excitations with no net (spin-independent) vorticity acting in the charge sector, which would not be subject to the problem in compressible superfluids, but these are found not to have fractional statistics. Note that a similar problem as for the charge sector (in the compressible case) occurs in the spin sector in connection with any subgroup of $\mathrm{SU}(2)$ that is spontaneously broken.

\section{EFFECTS OF DISORDER ON THE TRANSITIONS}

In this Section, we discuss the effects of disorder on the phases above, and on the transitions between them. We consider the phase-coherent, zero-temperature case, and neglect all interactions between the quasiparticles (including the gauge field fluctuations). The problem then reduces again to the quasiparticle effective Hamiltonians $K_{\text {eff }}$, this time with $\xi_{\mathbf{k}}$ and $\Delta_{\mathbf{k}}$ replaced by operators that are local in position space, with short-range correlations of the disorder. We consider the problems above in reverse order, starting with the d-wave case, which has the most symmetry ( $\mathrm{SU}(2)$ of spin rotations), then d- or p-wave with only U(1) symmetry, and finally the spinless p-wave case, with no continuous symmetry. The first case has been studied recently in the context of disordered superconductors 52 58, while we will argue that the second maps onto the usual noninteracting QHE transition, and the last includes an unusual intermediate phase where the heat transport is similar to that in a disordered metal. In all cases, we expect the qualitative results to be unaffected by interactions (or quantum fluctuations around the mean field theories used), though the universality classes may be changed.

The problem of the noninteracting BCS quasiparticles in disordered paired fermion systems was discussed by Altland and Zirnbauer (AZ) [52], where a symmetry classification of random matrix ensembles was proposed, that is analogous to the familiar classification for ordinary oneparticle Hamiltonians into orthogonal, unitary, and symplectic ensembles (or symmetry classes). In these ensembles, no particular value of the energy is singled out as special, so that they apply to phenomena near generic energy (or Fermi energy) values, where the average density of states is nonzero. The disordered paired systems differ first in that the fermion number is not conserved, because of the pairing terms. The full Hamiltonian of course conserves number, but this involves the collective response of the condensate. In applying these models in the FQHE, this is again true, but leads to the Hall response, not a superfluid response. Therefore we disregard particle number transport, and concentrate on conserved quantities carried by the quasiparticles only. The latter quantities include spin, when this symmetry is not broken spontaneously by the pairing. Then the classification of ensembles by AZ is according to whether time-reversal symmetry $(\mathrm{T})$ is broken or not, and whether or not there is an unbroken $\mathrm{SU}(2)$ spin rotation symmetry; the latter is unbroken in spin-singlet paired states. By making certain transformations, such as a particle-hole transformation on the $\downarrow$ spins in the cases where $S_{z}$ (at least) is conserved, the quasiparticle Hamiltonians can be related to number-conserving Hamiltonians, and thus to single-particle or random matrix problems. In this way, AZ identified four classes of random-matrix ensembles for disordered paired systems, which they labelled C, CI, D, DIII. For the quasiparticle Hamiltonians, the zero of quasiparticle energy $E$ is a special point, where in most cases the average density of states of the quasiparticles in the disordered system vanishes. Thus these four classes are distinct from the usual three mentioned above.

The case of spin-singlet paired states with disorder was considered in more detail [51,53,54]. The symme- 


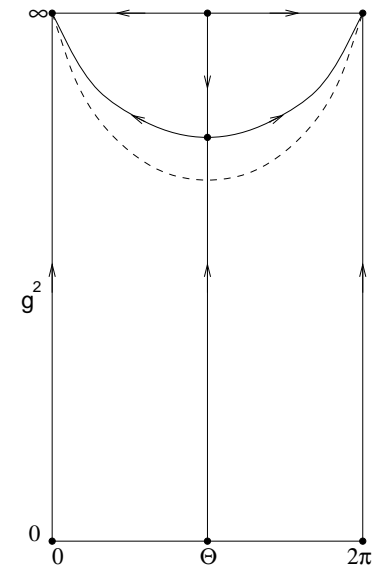

(a) class $\mathrm{C}$

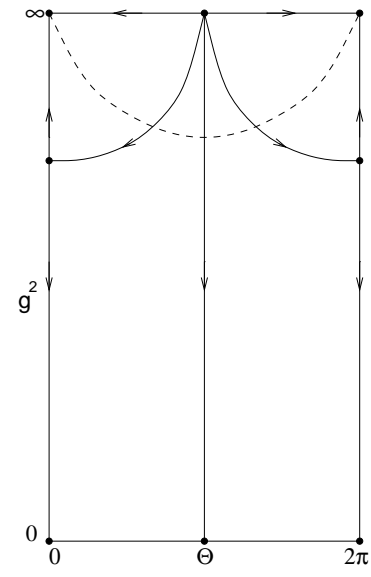

(b) class D
FIG. 2. Proposed renormalization group flow diagrams for (a) the unitary ensemble (IQHE), as in 96, and class C, and (b) class D. The dashed curves represent schematically the (nonuniversal) bare values of the coupling parameters. Other features are universal when the renormalized couplings are defined using the as-measured conductivity parameters, as explained in the text, and repeat periodically in the $\theta$ variable.

try classes are CI (with time reversal symmetry), and C (without time reversal). In the particular case of class C, it is natural to consider in two dimensions the possibility of a Hall spin conductivity $\sigma_{x y}^{s}$, as in the weak-pairing $\mathrm{d}$-wave phase [56,57. There are nonlinear sigma model formulations for class C, either using replicas, which lead to target manifold $\mathrm{Sp}(2 n) / \mathrm{U}(n)$ (with $n \rightarrow 0$ ) in the compact formulation [53], or supersymmetry, which leads to the target supermanifold $\operatorname{Osp}(2 n \mid 2 n) / \mathrm{U}(n \mid n)$ (with $n>0$ arbitrary) [52]. The Hall spin conductivity shows up in that a topological term with coefficient $\theta$ proportional to $\sigma_{x y}^{s}$ can be included in these models, when the dimension of space is two [53]. Analysis of the model, and numerical work on a network model with the symmetries appropriate to class $\mathrm{C}$ [56], has shown that this system has phases with quantized $\sigma_{x y}^{s}$ which are the same, or multiples of, those we discussed in the pure system in Subsec. IV . These phases can therefore be viewed as the disordered analogs of the weak- and strong-pairing phases. In these phases, the BCS quasiparticle (fermion) excitations at low energies have a non-zero density of states which vanishes quadratically as $E \rightarrow 0$, and these states are localized [55]. The situation is thus similar to the usual QHE, in which the low-energy excitations are localized and have nonvanishing density of states at the Fermi energy. Localization is necessary to obtain the quantized Hall conductivity. In the weak-pairing phase, we therefore expect that the results for the edge and statistics properties obtained in Sec. IV are still valid when disorder is included. We do not expect this conclusion to be affected by the inclusion of interactions in the analysis.

The transition between these two phases, and the role of $\sigma_{x y}^{s}$, can be understood via a renormalization group
(RG) flow diagram, shown schematically in Fig. 2 (a). The flows can be thought of as representing the values of the local conductivity parameters $\sigma_{x x}^{s}, \sigma_{x y}^{s}$ that would be measured at a given length scale, and how they change with this scale. In the nonlinear sigma model, similar parameters appear in the action of the quantum field theory. It is natural to define the renormalized values of these couplings at any scale to be the conductivities that would be measured at that scale, in which case the flows are the same as the RG flows within the field theory model. The conductivities should be understood in this way from here on, instead of as the bare values, with which they coincide only when the scale is of order the mean free path. Quantized values refer to the renormalized values at very large scales. In the case of class $\mathrm{C}$, the form of the flows is identical to that in the IQHE for noninteracting electrons 96. Similarly to the usual IQHE, the transition occurs because of a nontrivial fixed point, at which $\sigma_{x y}^{s}$ is midway between adjacent quantized values, and $\sigma_{x x}^{s}$ will take some nontrivial universal value. However, the spin quantum Hall transition in class $\mathrm{C}$ is in a different universality class from the IQHE. Recent numerical work has obtained some of the critical exponents for this transition, both from the network model [56], and from a mapping of the network model to a supersymmetric vertex model and a superspin chain [55,58,57], which was then analyzed numerically [57]. The results are in excellent agreement with exact values of some exponents, which were proposed using the relation of the supersymmetric vertex model to classical percolation [58]. For example, the localization length exponent is $\nu=4 / 3$, and at the critical point the density of states vanishes as $E^{1 / 7}$. The effect of interactions on these results is presently unknown.

Inclusion of a Zeeman splitting $\propto h$, which was neglected so far, will split the transition, and the phase diagram as a function of $\mu$ and $h$ will be similar to Fig. 4 in Ref. [57]. There will be an intermediate phase in which $\sigma_{x y}^{s}$ is quantized and halfway between the quantized values in the phases on either side. (This is somewhat like the intermediate MR phase produced by tunneling $t$ in the p-wave case.) In the present case, the Zeeman term leaves unbroken a $\mathrm{U}(1)$ subgroup of the $\mathrm{SU}(2)$ symmetry present in the spin-singlet paired state; for $\mathbf{h}$ parallel to the $z$ axis in spin space, this $\mathrm{U}(1)$ is generated by $S_{z}$. The pairing is still between spin $\uparrow$ and spin $\downarrow$, and so the symmetry is the same as in the p-wave case: with disorder, the distinction between p-wave and d-wave, and between spin-singlet and spin-triplet, is lost. Therefore we expect that in this intermediate phase, there is a single chiral Dirac fermion mode on the edge, and that the statistics properties are the same as those of the 331 state. Also, the transitions from the weak-pairing abelian phase, in which the statistics properties will be unchanged even though the $\mathrm{SU}(2)$ symmetry is broken (similar to the effect of $t$ on the 331 state), to this phase, and from this 
phase to the strong-pairing phase with $\sigma_{x y}^{s}=0$, are expected to be in the universality class of the usual IQHE [56 58]. Here the unbroken $\mathrm{U}(1)$ of spin is playing the role of the particle number in the usual IQHE case; the real particle number is of course still not conserved in the paired state. The appearance of this symmetry class in a disordered superconductor was seemingly overlooked by AZ; in fact, there are two such possible classes, in which there is an unbroken $\mathrm{U}(1)$ [not $\mathrm{SU}(2)]$, and T may be either unbroken or broken. By applying the methods used by AZ in the case of broken $\mathrm{T}$ and unbroken $\mathrm{U}(1)$ symmetry, one is led back to the usual unitary ensemble, with the spin $\uparrow$ and spin $\downarrow$ quasiparticles playing the role of particles and holes, respectively. It follows that these phases at non-zero $h$ possess a nonvanishing density of (localized) states for the quasiparticles at $E \rightarrow 0$. In the two-dimensional case, the unitary class admits a topological term with coefficient $\propto \sigma_{x y}^{s}$ in our case, and thus an IQHE transition. This is consistent with the results in Ref. [56]. Note that the relevant interaction effects in the case of paired states and the IQHE may turn out to be different, however, so the equivalence might not hold when interactions are taken into account.

Turning to the p-wave states relevant to the doublelayer system in the FQHE, for $t=0$ and with nonzero disorder we are once again in the situation just discussed of broken $\mathrm{T}$ and unbroken $\mathrm{U}(1)$. Hence for this case, we again expect, within our model of noninteracting quasiparticles, a transition between quantized $\sigma_{x y}^{s}$ phases (analogs of the weak and strong-pairing phases) that is in the universality class of the noninteracting IQHE. With $t \neq 0$, we saw in the pure case that the transition splits into two, each in the class of the spinless p-wave case, and the intermediate phase had the properties of the MR state. The non-zero tunneling breaks the U(1) symmetry. We consider this case next, and then return to its application to the double-layer system.

The symmetry classes of pairing of spinless fermions, or with $\mathrm{SU}(2)$ symmetry fully broken by the Hamiltonian, are denoted DIII (with unbroken T) and D (with broken T) by AZ. These cases were not analyzed in two dimensions previously, but some of our results have been found independently in Ref. 97 (see also a remark in Ref. [54]). Our interest is in class $\mathrm{D}$, with broken $\mathrm{T}$. In this case the nonlinear sigma model target (super) manifold is $\mathrm{SO}(2 n) / \mathrm{U}(n)$ (using replicas in the compact formulation, with $n \rightarrow 0)$, or $\operatorname{Osp}(2 n \mid 2 n) / \mathrm{U}(n \mid n)$ using supersymmetry (with $n>0$ arbitrary) [52]. In the supersymmetric formulations, classes $\mathrm{C}$ and $\mathrm{D}$ differ in that, while the bosonic submanifolds are real forms of $\mathrm{SO}(2 n) / \mathrm{U}(n) \times \mathrm{Sp}(2 n) / \mathrm{U}(n)$ in both cases, in class $\mathrm{C}$ the first factor is noncompact and the second compact, and in class $\mathrm{D}$ it is the other way round (corresponding to the compact replica formulation) 52. (These statements are for $n>1$. For $n=1$, the first factor is a single point in both cases.) In the two-dimensional case, class D admits a topological term in the nonlinear sigma model, like class C. In the case of class D, there is no continuous symmetry in the underlying fermion problem. The only candidates for the physical meaning of the couplings in the nonlinear sigma model are in terms of thermal conductivities, since energy is still a conserved quantity [53]. The diagonal (dissipative) thermal conductivity $\kappa_{x x}=\kappa_{y y}$, and the off-diagonal LR conductivity $\kappa_{x y}$ have the dimensions of $k_{B}^{2} T / h$ ( $h$ is Planck's constant, $=2 \pi$ in our units) at low temperatures. We define

$$
\begin{gathered}
\kappa_{x x}=\frac{\pi^{2} k_{B}^{2} T}{3 h} \tilde{\kappa}_{x x}, \\
\kappa_{x y}=\frac{\pi^{2} k_{B}^{2} T}{3 h} \tilde{\kappa}_{x y},
\end{gathered}
$$

where the numerical factor of $\pi^{2} / 3$ is that which arises in the quantized values of $\kappa_{x y}$ (see Sec. IV (), and so may be conveniently included here. Then we expect that the sigma model couplings are (similarly to the charge and spin transport cases, where no factors of $k_{B}^{2} T$ are involved) $1 / g^{2} \sim \tilde{\kappa}_{x x}$, and $\theta=4 \pi \tilde{\kappa}_{x y}$. Since the quantized values of $\tilde{\kappa}_{x y}$ are $\tilde{\kappa}_{x y}=c$, which is a multiple of $1 / 2$ for Majorana fermions, we have arranged that the quantized values of $\theta$ would be multiples of $2 \pi$. We expect that if the nonlinear sigma model for class $\mathrm{D}$ is derived microscopically at weak coupling, which we will not attempt here, then the above relation for $\theta$ will hold. We note here that in class $\mathrm{D}$, the density of states at $E \rightarrow 0$ in the localized (quantized $\tilde{\kappa}_{x y}$ ) phases is expected to approach a nonzero nonuniversal constant, as one can see from the random matrix expressions in AZ [52], using an argument in Ref. [55. We want to emphasise that the nonlinear sigma model in class D describes only the case of very generic disorder, and not necessarily more restricted forms of disorder. We will return to this point below.

We may now consider the form of the RG flows for class $\mathrm{D}$ in two dimensions. We begin with perturbation theory at weak coupling, that is large $\tilde{\kappa}_{x x}$. This was considered previously for classes C and CI [51,53], and for class D is mentioned in Ref. [54]. We define the RG beta function as

$$
\beta_{x x}\left(g^{2}\right) \equiv \frac{d\left(g^{2}\right)}{d \ln L},
$$

where $g^{2}$ is the nonlinear sigma model coupling squared, and $g^{2} \sim 1 / \tilde{\kappa}_{x x}$, and $L$ is the length scale on which the renormalized coupling is defined. In two dimensions this has the form

$$
\beta_{x x}\left(g^{2}\right)=a g^{4}+O\left(g^{6}\right)
$$

at weak coupling (small $g^{2}$ ); effects of the topological term involving $\theta$, if the model admits one, are nonperturbative in $g^{2}$, of order $e^{-b / g^{2}}$, and contain the $\theta$ dependence. (Also, there will be another beta function 
$\beta_{x y} \equiv d \theta / d \ln L$ for $\theta$, which will be entirely nonperturbative.) Here $a$ and $b$ are constants. In classes $\mathrm{C}$ and CI, $a$ is positive [51,53], and the flow is towards strong coupling, that is localization, as shown in Fig. 2 (a) for class C. However, in classes D and DIII, a is negative, and in fact equal to minus its values in $\mathrm{C}$ and CI respectively [54,98]. The reason lies in the relation of the target (super-)manifolds in the nonlinear sigma models, described above. This relation is similar to that between the manifolds in the symplectic (spinorbit scattering) and orthogonal (potential scattering) ensembles of the usual random matrix or localization problems, where the $a$ 's also have opposite signs. The origin of this is that the $a$ term in the beta function of any nonlinear sigma model is related to the Ricci curvature tensor of the target manifold. When we compare these for the compact and noncompact versions of the "same symmetry", such as $\operatorname{Sp}(2 n) / \mathrm{U}(n)$ (at $n$ a positive integer), we find that they are of opposite sign: the noncompact case has negative curvature, the compact positive. These geometric phenomena for symmetric Riemannian (non-super-) manifolds are discussed by Helgason [99]. The noncompact space $\operatorname{Sp}(2 n, \mathbf{R}) / \mathrm{U}(n)$ at $n \rightarrow 0$ represents class $\mathrm{D}$ in perturbation theory, as does the compact space $\mathrm{SO}(2 n) / \mathrm{U}(n)$ in the same limit, and so their perturbative $\beta$ functions are equal. This establishes the result using replicas. Likewise for the target supermanifolds, the factors in the bosonic parts each have opposite curvature in the two cases, and this presumably extends to the full supermanifold. Consequently, $a$ has the opposite sign in the two cases. A similar result also holds in the principal chiral models with target spaces $\mathrm{Sp}(2 n)$ and $\mathrm{SO}(2 n)$ in the compact replica approach, with $n \rightarrow 0$, which describe classes CI and DIII respectively. Note that in the unitary case the target supermanifold is $\mathrm{U}(n, n \mid 2 n) /[\mathrm{U}(n \mid n) \times \mathrm{U}(n \mid n)]$, and the bosonic part contains just the compact and noncompact forms of $\mathrm{U}(2 n) /[\mathrm{U}(n) \times \mathrm{U}(n)]$, so the model maps to itself under interchange of compact and noncompact, and hence the net coefficient $a$ in the unitary case vanishes, as is well-known.

We now try to find the simplest possible flow diagram compatible with the weak-coupling behavior and some other simple requirements. In the unitary case, the flows in Fig. 2 (a) can be considered to be the simplest possible, on including nonperturbative $\theta$-dependent effects that cause the attractive fixed points to be at $\theta \equiv 0(\mathrm{mod}$ $2 \pi$ ). These flows are in fact obtained if one takes the dilute instanton gas calculations of Pruisken, which are the leading nonperturbative effects at weak coupling, together with the perturbation theory result just discussed, and uses these forms for all coupling values [96]. The nontrivial fixed point at $\theta=\pi$ and at some universal $\sigma_{x x}$ controls the transition between the quantized fixed points in this case. The picture obtained from these flows seems to be in qualitative agreement with what is known from numerical work for this transition in the unitary ensemble, and for class C [56].

For class $\mathrm{D}$ we can try to guess the nonperturbative form of the flows without calculation. In view of the weak-coupling result, we could try reversing the arrows on the flows for unitary and class C. However, we also expect that the stable, attractive fixed points, which will represent quantized values of $\tilde{\kappa}_{x y}$ at $\tilde{\kappa}_{x x}=0$, will be at $\theta \equiv 0(\bmod 2 \pi)$ again, not at $\pi(\bmod 2 \pi)$. In particular, this means that an insulating phase with quantized $\tilde{\kappa}_{x y}=$ 0 is possible. It seems reasonable that sufficiently small $\tilde{\kappa}_{x x}$ can produce localization when $\tilde{\kappa}_{x y}=0$, in spite of the flow to $\tilde{\kappa}_{x x}=\infty$ in the weak-coupling region, just as in other localization problems, including the case of spinorbit scattering, and this should be stable against small changes in $\tilde{\kappa}_{x y}$. In order to achieve this, we also shift the flows by $\pi$ along the $\theta$ direction. The result is the flow diagram shown in Fig. 2 (b). The interesting nontrivial fixed points now occur on the lines $\theta \equiv 0(\bmod$ $2 \pi$ ). These flows could be checked in the weak-coupling region by comparing them with a dilute instanton gas calculation as in the unitary case. Indeed, if the latter calculation is assumed to give the same form as in the unitary case [96], as is plausible, then the competition with the perturbative terms will give the flows as shown.

In order to use the RG flow diagram to make predictions about the effects of disorder on the pure transition from weak to strong pairing with $\mathrm{T}$ broken and no spin-rotation symmetry, we need to know where the bare values of $\tilde{\kappa}_{x x}$ and $\tilde{\kappa}_{x y}$ lie on the diagram. In the usual IQHE unitary case, and also for class C, the values are shown as the dashed curve in Fig. 2 (a). If one uses the self-consistent Born approximation to obtain the values in the IQHE case, for disorder weak compared with the cyclotron energy, then one obtains a semicircle in the $\sigma_{x x^{-}}$ $\sigma_{x y}$ plane [96. The precise position of the curve is unimportant, but it associates the transition, at which $\sigma_{x y}$ is half an odd integer, with the middle of the disorderbroadened Landau bands. Similar behavior occurs for class C.

In the present case of class $\mathrm{D}$, we again expect the bare values of $\tilde{\kappa}_{x x}$ and $\tilde{\kappa}_{x y}$ to lie on an arc, as shown in Fig. 2 (b). These values pass through the quantized points at $\tilde{\kappa}_{x x}=0\left(g^{2}=\infty\right)$ and $\theta \equiv 0(\bmod 2 \pi)$ (quantized $\left.\tilde{\kappa}_{x y}\right)$. This is reasonable, as these are the values in the two phases in the pure case, and disorder that is weak compared with the gap $|\mu|$ in the spectrum should produce only small corrections to these values. Connecting these regions with the dashed arc, we always produce a curve of the form shown for topological reasons. This curve intersects the separatrices shown, which flow into the non-trivial fixed points. We see that the regions near the quantized fixed points flow into those fixed points, so that the quantized phases, one of which corresponds to the nonabelian statistics phase in the pure case, still exist in the presence of disorder according to our proposed 
flow diagram. But there is an intermediate set of bare values near $\theta \equiv \pi(\bmod 2 \pi)$, which flow to weak coupling, and at large scales they map onto the entire interval of $\tilde{\kappa}_{x y}$ values between the two quantized values in question, with a $\tilde{\kappa}_{x x}$ that increases logarithmically with $L$, according to the weak coupling beta function $\beta_{x x}$ above. This is therefore an intermediate phase with metallic behavior of the thermal conductivities, between the two quantized phases. The intermediate phase is separated by phase boundaries from the quantized phases, and the critical behavior at these transitions is governed by the nontrivial fixed points. At these fixed points, $\tilde{\kappa}_{x y}$ is equal to the quantized value in the neighboring quantized phase. The critical exponents for these transitions are unknown at present. Experimentally, one would see plateaus in $\tilde{\kappa}_{x y}$, separated by intermediate regions, and the width of the latter will stay nonzero as the system size goes to infinity, and as the temperature goes to zero. In the intermediate regions, $\tilde{\kappa}_{x y}$ will vary continuously to interpolate its neighboring quantized values, and $\tilde{\kappa}_{x x}$ will have a peak, the height of which will grow logarithmically with increasing system size or inverse temperature. We emphasize that the charge transport properties are still either superconducting or quantized Hall, depending on the system considered, and unaffected by the transition in the quasiparticles (there would be a collective mode (phonon-like) contribution to thermal transport in a neutral superfluid case, such as a $\mathrm{He}^{3}$ film).

We should respond to one possible objection to our claim that there will generically be an intermediate metallic phase in the class D problem (this point is raised in Ref. 97]). This objection begins with the pure problem, in which (since we assume noninteracting quasiparticles) the critical theory is a Majorana fermi field with a mass term that changes sign, and then considers weak disorder as a perturbation of this continuum theory. The similar problem of a Dirac field has been analyzed in recent years [100], and a central argument is that for $E=0$, one can consider the problem as a Dirac field in two (Euclidean) dimensions. It is then argued that there are only a few possible random terms that can be included in the 2D Dirac action that are marginal or relevant by power counting at the pure fixed point. These terms, which are bilinears in the Dirac field since the problem is noninteracting, are a random $\mathrm{U}(1)$ vector potential and two types of random mass term. For the random Majorana fermion, no $\mathrm{U}(1)$ vector potential is possible, and there is a unique mass term. It is further known that the mass term is marginally irrelevant for weak disorder; this type of randomness arises when one considers the 2D Ising model with disorder in the intersite Ising couplings [101]. If one assumes that the disordered paired system we consider here must fall into this scheme, then the only possible randomness is irrelevant, and there will be a transition directly between the disordered versions of the weak- and strong-pairing phases, with the critical properties of the pure system, up to logarithmic corrections, in disagreement with our prediction of an intermediate phase and a different universality class.

There is, however, a form of disorder not considered in this argument. In Sec. II, we discussed vortices in the gap function, and in Subsec. II g related their description in terms of the gap function and the vector potential of the underlying problem to the vielbein and spin connection that appear in the general Majorana action. We should consider the possibility that these are random; indeed, the general analysis of $\mathrm{AZ}$ requires generic randomness, even though the Dirac or Majorana actions do not explicitly appear there. Not all of the random fluctuations of these quantities are necessarily relevant. For example, small random fluctuations of the magnitude of the vielbein (i.e., of $|\hat{\Delta}|$ ) around its non-zero mean are irrelevant by power counting. Also, if the gap function is nonzero the vector potential can mostly be gauged away because the superconductor is a Meissner phase. However, this is clearly not true at a vortex, and for general probability distributions of the disorder, vortices will be present. In the FQHE application, there is underlying potential disorder which couples to the particle density, and for unbounded distributions of disorder, or for sufficiently strong disorder with a bounded distribution, it will nucleate vortices (FQHE quasiparticles) in the ground state, which can occur in isolation from other vortices since they have finite energy. It is not difficult to see, either intuitively or formally, that a small density of randomly placed vortices will be highly relevant at the massless Majorana critical point. Intuitively, they introduce destructive phase interference. Formally, in the 2D Majorana action, the effect is to insert the spin fields of the Majorana theory (so-called because they represent the Ising spins in the related Ising model), which (in the same gauge choice as earlier) cause square-root branch points in the Majorana field 92]. Such random vortices do not seem to have been considered in previous work on random Dirac fields. They are relevant because, while the spin field in the critical Majorana theory has scaling dimension $1 / 8$ (which corresponds to a relevant perturbation even in the non-random case), on replicating or supersymmetrizing the system, the spin fields act on all the components simultaneously, and hence their dimension is then $0 \times 1 / 8=0$. Thus the coefficient with which these fields appear in the action after averaging has scaling dimension 2, showing they are strongly relevant [98. This will cause an RG flow away from the pure Majorana fixed point, and we expect the generic behavior of class $\mathrm{D}$, with the intermediate metallic phase, to result. Another possible form of disorder is that which violates the $l=-1$ symmetry, that is general $\mathrm{p}_{x}+i \mathrm{p}_{y}$ pairing with random coefficients. If both were completely random, the average would restore parity, that is the symmetry of reflection in any line, and prevent the existence of nonzero $\tilde{\kappa}_{x y}$, so this form of disorder is not completely acceptable 
in our physical systems; we must allow for a net violation of parity. Thus, the generic disorder that defines class D should include all of these relevant effects, and randomly placed, isolated vortices alone are a relevant perturbation that leads to a flow away from the pure Majorana critical theory. Notice that, with random vortices included, the ordered phase we are describing is no longer a superconductor in the strict sense, due to the random phases frozen into the gap function. Instead it is what has been called a gauge glass, since the order in the gap function is similar to that in a spin glass.

In contrast, in the random bond Ising model (a subject also raised in Ref. 97), negative bonds produce frustrated plaquettes, and a string of negative bonds produces two frustrated plaquettes at the ends of the string. The insertion of a semi-infinite string of negative bonds is the definition of the Ising disorder operator of Kadanoff and Ceva [102], which is dual to, and at the critical point has the same scaling dimension as, the Ising spin field. However, in this case the analysis of weak randomness in terms of a random mass is justified, because for a low probability of negative bonds the disorder variables (vortices) appear only in closely-spaced pairs, not in isolation. In the continuum critical field theory, the operator product of two disorder operators, which represents the close pair, produces the Majorana mass operator, and thus the randomness generates the random mass term. We see that the distribution of the randomness in the Ising problem with weak bond disorder differs substantially from the problem we wish to consider. While the random bond Ising model in $2 \mathrm{D}$ does have a direct transition between the two quantized $\tilde{\kappa}_{x y}$ phases, and a multicritical point, there is no reason to suppose that these occur in class D.

The properties of the disordered version of the MR phase, which has a nonzero quantized $\tilde{\kappa}_{x y}$, are subtle. Since we have assumed that isolated vortices are possible, which are localized FQHE quasiparticles analogous to those in the usual states on the plateaus in the FQHE, these will carry zero modes, and there will be $2^{n-1}$ manyparticle states when the system contains $2 n$ vortices. These are very nearly degenerate when the vortices are well-separated, since the energy splittings are expected to decrease exponentially in the separation of the vortices. Nonabelian statistics of additional vortex (FQHE quasiparticle) excitations should be considered in terms of exchanges of such quasiparticles separated by many times the typical separation of the vortices in the ground state. Then the fermion zero modes of the ground state can interfere with those on the added quasiparticles, complicating the nonabelian statistics properties. Further study of these effects is beyond the scope of this paper.

Finally, we return as promised to the case of the double-layer system with tunneling $t$. Then the phase boundaries at non-zero $t$ between the weak-pairing, abelian phase, the MR phase, and the strong-pairing phase will be broadened and replaced by an intermediate region in which metallic thermal properties will occur, again with sharp phase boundaries between this and the other phases. As $t \rightarrow 0$, this intermediate phase will shrink in width to become a single point at $t=0$, where we have already explained that a direct transition in the IQHE universality class occurs. Thus this transition broadens to become the intermediate metallic phase at finite $t$. We expect that at sufficiently small $t$, there is a single region of the metallic phase, which interpolates between $\tilde{\kappa}_{x y}$ values differing by two steps, which are the values in the $t=0$ weak and strong-pairing phases. As $t$ increases, a point is reached at which another plateau in $\tilde{\kappa}_{x y}$ appears, which is the MR-type phase. Such behavior is allowed by our flow diagram, if we plot it from $\theta=0$ to $\theta=4 \pi$, and the initial values lie on an arc between the quantized fixed points at those $\theta$ values that avoids the basin of attraction of the quantized fixed point at $\theta=2 \pi$ completely for small $t$, but not for larger $t$.

\section{CONCLUSION}

In this paper, we have considered exotic properties of $\mathrm{P}$ and T-violating paired states of fermions in two dimensions, and the relation to the FQHE using pairing of composite fermions. The results have been summarized in the Introduction.

To conclude, our main results are: (i) p-wave pairing in spinless or spin-polarized fermions in the weak-pairing phase leads to the properties also found in the FQHE in the MR states, and supports the ideas of nonabelian statistics as a robust property, at least in the case of a pure system. Such statistics will also occur for the vortices in such a p-wave state in general in charged superfluids, and in neutral superfluids modulo $\mathrm{U}(1)$ phase factors that arise from the compressible charge sector; (ii) in an A-phase type p-wave phase, statistics may be abelian, though tunneling or a Zeeman term may lead to a transition to a MR phase; (iii) in the d-wave spinsinglet case, the HR state corresponds to the transition point, and, from now on, may be disregarded in considering generic spin-singlet FQHE systems, which will most likely be in the weak-pairing phase. The latter is abelian, but has a nonzero Hall spin conductivity, and spin-1/2 chiral Dirac fermions on the edge [57]; (iv) disorder does not destroy the phases in question, but may modify the $\mathrm{MR}$ phase in an essential way. In the spinless p-wave case, randomly-placed vortices are a relevant perturbation of the pure transition, and there is an intermediate phase with metallic thermal conductivity properties due to delocalized BCS quasiparticles.

Issues which remain to be addressed include the full effective theories for the states, and for the transitions between them, and the effect of interactions on the random problems. Also, a direct derivation of nonabelian 
statistics in terms of pairing of fermions in the MR case is desirable.

One further comment on tunneling into the edge is in order. Such tunneling could provide a useful diagnostic for the paired states in the FQHE. Since the fermion excitations on the edge in the weak-pairing phases are now always Dirac (or Majorana), their contribution to the exponent is always the same. Thus at filling factor $\nu=1 / q$ (where $q$ will be $2,4, \ldots$, for fermions such as electrons), the current will scale as $I \sim V^{\alpha}$ with $\alpha=q+1$ in all the weak-pairing or MR phases. In contrast, in the Halperin-type paired states, we have $\alpha=4 q$ [44]. The former result is the same as in the compressible Fermiliquid-like states 103. Assuming that edge theories of fully-gapped bulk states must always be unitary conformal field theories, as argued in Sec. IV, the exponent for tunneling into an edge on which all modes propagate in the same direction must in fact always be an odd integer, as a consequence of the Fermi statistics of the electrons. For the $5 / 2$ state, the $I \sim V$ contribution of the LLL will presumably dominate.

Note Added: In view of a suggestion which has circulated, that the Fermi-liquid-like phase of Ref. 25] may generically have an instability to pairing in some channel, albeit at extremely low energy or temperature scales, we will consider here the case of a weak-pairing phase of spinpolarized fermions in an arbitrary angular momentum $l$ eigenstate ( $l$ must be odd). Similar arguments as before show that there will be $|l|$ chiral Majorana fermion modes on an edge, and correspondingly $2^{|l| n-1}$ degenerate states for $2 n$ vortices. Since $|l|$ must be odd, this always leads to nonabelian statistics, with the same monodromy properties as for $l=-1$ up to Berry phase factors (because each added pair of Majoranas makes a Dirac fermion which contributes only abelian effects).

\section{ACKNOWLEDGMENTS}

We thank M.P.A. Fisher, I.A. Gruzberg, F.D.M. Haldane, B.I. Halperin, T.-L. Ho, A.W.W. Ludwig, M.V. Milovanović, G. Moore, E.H. Rezayi, and T. Senthil for informative comments and helpful discussions. N.R. is grateful for the hospitality of the Institut Henri Poincaré, Paris, where this paper was completed. This work was supported by NSF grants, numbers DMR-9157484 and DMR-9818259.

\section{APPENDIX A: QUANTIZED HALL CONDUCTIVITY FOR SPIN}

In this Appendix, we provide a detailed derivation of the Hall conductivity in (iso-) spin transport in the d- and p-wave (A-phase) cases. This is equivalent to showing that the induced action for the system in an external gauge field that couples to the (iso-) spin is a CS term. In the d-wave case, the system is spin-rotation invariant, so we obtain an $\mathrm{SU}(2) \mathrm{CS}$ term, while in the p-wave case, there is only a $\mathrm{U}(1)$ symmetry, so we find a $\mathrm{U}(1)$ CS term. In both cases, the Hall spin conductivity is given in suitable units by a topological invariant. Within the BCS mean field approach, using a suitable conserving approximation, this topological invariant is the winding number of $\left(u_{\mathbf{k}}, v_{\mathbf{k}}\right)$ discussed in Sec. II], and is therefore an integer, which is the statement of quantization. We argue that the quantization in terms of a topological invariant is more general than the approximation used.

Considering first the spin-singlet paired states, we use the Nambu basis where the symmetries are transparent. Define

$$
\Psi=\frac{1}{\sqrt{2}}\left(\begin{array}{c}
c \\
i \sigma_{y} c^{\dagger}
\end{array}\right)
$$

with

$$
c=\left(\begin{array}{c}
c_{\uparrow} \\
c_{\downarrow}
\end{array}\right)
$$

so that $\Psi$ transforms as a tensor product of particle-hole and spin-space spinors. We consider an interacting system and approximate it as in BCS theory, then with a minimal coupling to the gauge field, we use a conserving approximation to obtain the spin response. In Fourier space, we should note that

$$
\Psi_{\mathbf{k}}=\frac{1}{\sqrt{2}}\left(\begin{array}{c}
c_{\mathbf{k}} \\
i \sigma_{y} c_{-\mathbf{k}}^{\dagger}
\end{array}\right)
$$

In the Nambu basis, the kinetic energy becomes (again, $K=H-\mu N)$

$$
\begin{aligned}
K_{0} & =\sum_{\mathbf{k}} \xi_{\mathbf{k}}^{0}\left(c_{\mathbf{k} \uparrow}^{\dagger} c_{\mathbf{k} \uparrow}+c_{\mathbf{k} \downarrow}^{\dagger} c_{\mathbf{k} \downarrow}\right) \\
& =\sum_{\mathbf{k}} \xi_{\mathbf{k}}^{0} \Psi_{\mathbf{k}}^{\dagger}\left(\sigma_{z} \otimes I\right) \Psi_{\mathbf{k}},
\end{aligned}
$$

where $\xi_{\mathbf{k}}^{0}=|\mathbf{k}|^{2} /(2 m)-\mu$ is the kinetic energy, containing the bare mass $m$, and the products in the spinor space are understood. Products like $\sigma_{z} \otimes I$ act on the Nambu spinors, with the first factor acting in the particle-hole factor, the second in the spin-space factor. The interaction term, for a spin-independent interaction $V$, is

$$
K_{\mathrm{int}}=\frac{1}{2} \sum_{\mathbf{k k}^{\prime} \mathbf{q}} V_{\mathbf{q}}: \Psi_{\mathbf{k}+\mathbf{q}}^{\dagger}\left(\sigma_{z} \otimes I\right) \Psi_{\mathbf{k}} \Psi_{\mathbf{k}^{\prime}-\mathbf{q}}^{\dagger}\left(\sigma_{z} \otimes I\right) \Psi_{\mathbf{k}^{\prime}}:
$$

Here the colons : ... : denote normal ordering, that is all the $c^{\dagger}$ s are brought to the left. In the BCS-extended Hartree-Fock approximation, the effective quasiparticle Hamiltonian (for later reference) is 


$$
\begin{aligned}
K_{\mathrm{eff}}= & \sum_{\mathbf{k}} \Psi_{\mathbf{k}}^{\dagger}\left[\xi_{\mathbf{k}}\left(\sigma_{z} \otimes I\right)+\operatorname{Re} \Delta_{\mathbf{k}}\left(\sigma_{x} \otimes I\right)\right. \\
& \left.-\operatorname{Im} \Delta_{\mathbf{k}}\left(\sigma_{y} \otimes I\right)\right] \Psi_{\mathbf{k}} .
\end{aligned}
$$

This is for singlet pairing, where $\Delta_{-\mathbf{k}}=\Delta_{\mathbf{k}}$, and not just for d-wave. Here $\xi_{\mathbf{k}}$ is $\xi_{\mathbf{k}}^{0}$ plus the Hartree-Fock corrections. If we define a vector

$$
\mathbf{E}_{\mathbf{k}}=\left(\operatorname{Re} \Delta_{\mathbf{k}},-\operatorname{Im} \Delta_{\mathbf{k}}, \xi_{\mathbf{k}}\right)
$$

then the quasiparticle energy $E_{\mathbf{k}}=\left|\mathbf{E}_{\mathbf{k}}\right|$, and

$$
K_{\mathrm{eff}}=\sum_{\mathbf{k}} \Psi_{\mathbf{k}}^{\dagger}\left(\mathbf{E}_{\mathbf{k}} \cdot \boldsymbol{\sigma} \otimes I\right) \Psi_{\mathbf{k}} .
$$

In the Nambu notation, it is clear that $K=K_{0}+K_{\text {int }}$, and $K_{\text {eff }}$, are invariant under global SU(2) rotations that act on the spin-space, that is the second factor in the tensor products. The spin density, the integral of which over all space is the total spin and generates such global transformations, and the spin current densities are given by

$$
\begin{aligned}
& J_{0}^{a}(\mathbf{q})=\frac{1}{2} \sum_{\mathbf{k}} \Psi_{\mathbf{k}-\mathbf{q} / 2}^{\dagger}\left(I \otimes \sigma_{a}\right) \Psi_{\mathbf{k}+\mathbf{q} / 2} \\
& J_{i}^{a}(\mathbf{q})=\frac{1}{2} \sum_{\mathbf{k}} \frac{k_{i}}{m} \Psi_{\mathbf{k}-\mathbf{q} / 2}^{\dagger}\left(\sigma_{z} \otimes \sigma_{a}\right) \Psi_{\mathbf{k}+\mathbf{q} / 2}
\end{aligned}
$$

where $i=x, y$ is the spatial index, and $a=x, y, z$ is the spin-space index. Spin conservation implies the continuity equation, as an operator equation,

$$
\partial J_{\mu}^{a} / \partial x_{\mu}=0,
$$

where $\mu=0, x, y$, and the summation convention is in force.

So far we have not introduced a gauge field for spin. Since the spin is conserved locally, we can turn the symmetry into a local gauge symmetry by introducing an $\mathrm{SU}(2)$ vector potential, and making all derivatives covariant. The effect on $K$ is to add the integral of

$$
A_{\mu}^{a} J_{\mu}^{a}+\frac{1}{8 m} A_{i}^{a} A_{i}^{a} \Psi^{\dagger}\left(\sigma_{z} \otimes I\right) \Psi .
$$

The gauge field is to be used solely as an external source, with which to probe the spin response of the system, and then set to zero.

If we now consider integrating out the fermions, then we can obtain an action in the external gauge fields, which can be expanded in powers of $A_{\mu}^{a}$. The zerothorder term is the free energy density, times the volume of spacetime, and the first-order term vanishes by spinrotation invariance. The second-order term corresponds to linear response: the second functional derivative with respect to $A_{\mu}^{a}$, at $A_{\mu}^{a}=0$, yields the (matrix of) linear response functions. In particular, the spatial components yield the conductivity tensor in the usual way. Therefore we consider the imaginary-time time-ordered function,

$$
\Pi_{\mu \nu}^{a b}=-i\left\langle J_{\mu}^{a}(q) J_{\nu}^{b}(-q)\right\rangle,
$$

where time-ordering is understood, and from here on we use a convention that $p, q$, etc stand for three-vectors $p=\left(p_{0}, \mathbf{p}\right)$, and further $p_{0}=i \omega$ is imaginary for imaginary time. For $\mu=\nu=i=x$ or $y$, an additional "diamagnetic" term $\bar{n} \delta^{a b} / 4 m$ is present in $\Pi_{\mu \nu}^{a b}$, which we do not show explicitly. As consequences of the continuity equation and the related gauge invariance, $\Pi_{\mu \nu}$ must be divergenceless on both variables, $q_{\mu} \Pi_{\mu \nu}=q_{\nu} \Pi_{\mu \nu}=0$. To maintain these when using the BCS-Hartree-Fock approximation for the equilibrium properties, one must use a conserving approximation for the response function, which in this case means summing ladder diagrams (compare the charge case in Ref. [2], pp. 224-237).

One begins with the BCS-Hartree-Fock approximation, which can be written in terms of Green's functions as (we consider only zero temperature, and $\int d p_{0}$ is along the imaginary $p_{0}$ axis throughout)

$$
\begin{aligned}
G^{-1}(p) & =p_{0}-\xi_{\mathbf{p}}^{0} \sigma_{z} \otimes I-\Sigma(p), \\
\Sigma(p) & =i \int \frac{d^{3} k}{(2 \pi)^{3}}\left(\sigma_{z} \otimes I\right) G(k)\left(\sigma_{z} \otimes I\right) V(k-q) .
\end{aligned}
$$

Note that $G(p)$ and $\Sigma(p)$ are matrices acting on the tensor product space. The equations are solved by

$$
G^{-1}(p)=p_{0}-\mathbf{E}_{\mathbf{p}} \cdot \boldsymbol{\sigma} \otimes I,
$$

(we write 1 for $I \otimes I$ ) as one can also see from the effective quasiparticle Hamiltonian $K_{\text {eff }}$, and $\Delta_{\mathrm{p}}$ obeys the standard gap equation.

In the response function, the ladder series can be summed and included by dressing one vertex, to obtain (again not showing the diamagnetic term)

$$
\begin{aligned}
\Pi_{\mu \nu}^{a b}(q)= & -i \int \frac{d^{3} p}{(2 \pi)^{3}} \operatorname{tr}\left[\gamma_{\mu}^{a}(p, p+q) G(p+q)\right. \\
& \left.\times \Gamma_{\nu}^{b}(p+q, p) G(p)\right]
\end{aligned}
$$

where $\gamma_{\mu}^{a}$ is the bare vertex,

$$
\begin{aligned}
& \gamma_{0}^{a}(p, p+q)=\frac{1}{2} I \otimes \sigma_{a}, \\
& \gamma_{i}^{a}(p, p+q)=-\frac{\left(p+\frac{1}{2} q\right)_{i}}{2 m} \sigma_{z} \otimes \sigma_{a},
\end{aligned}
$$

and $\Gamma_{\mu}^{a}$ is the dressed vertex satisfying

$$
\begin{aligned}
\Gamma_{\nu}^{b}(p+q, p)= & \gamma_{\nu}^{b}(p+q, p)+i \int \frac{d^{3} k}{(2 \pi)^{3}} \sigma_{z} \otimes I G(k+q) \\
& \times \Gamma_{\nu}^{b}(k+q, k) G(k) \sigma_{z} \otimes I V(p-k) . \quad(\mathrm{A}
\end{aligned}
$$

At small $q$, we can obtain useful information about this function from the Ward identity that results from the 
continuity equation. The particular Ward identity we use here is an exact relation of the vertex function to the self-energy, and the conserving approximation (the ladder series) was constructed to ensure that it holds also for the approximated vertex and self energy functions.

Following Schrieffer's treatment [2], we consider the vertex function with external legs included:

$$
\Lambda_{\mu}^{a}\left(r_{1}, r_{2}, r_{3}\right)=\left\langle J_{\mu}^{a}\left(r_{3}\right) \Psi\left(r_{1}\right) \Psi^{\dagger}\left(r_{2}\right)\right\rangle,
$$

for spacetime coordinates $r_{1}, r_{2}, r_{3}$. Applying $\partial / \partial r_{3 \mu}$ to both sides and using the operator continuity equation, we obtain the exact identity in Fourier space

$$
q_{\mu} \Gamma_{\mu}^{a}(p+q, p)=\frac{1}{2} I \otimes \sigma_{a} G^{-1}(p)-\frac{1}{2} G^{-1}(p+q) I \otimes \sigma_{a} .
$$

Since $G^{-1}$ is trivial in the spin-space indices, it commutes with $I \otimes \sigma_{a}$. Hence at $q \rightarrow 0$, the right-hand side vanishes, so $\Gamma(p+q, p)$ has no singularities as $q \rightarrow 0$. This differs from the charge case, for example, where this calculation (using the ladder series approximation) leads to the discovery of the collective mode [63]. Since the spin symmetry is unbroken, no collective mode is necessary to restore this conservation law, and so there is no singularity in the vertex function for spin.

One can verify that the Ward identity is satisfied using the BCS-Hartree-Fock $G^{-1}$ and the ladder series for $\Gamma$. At $q=0$, this yields the important results

$$
\Gamma_{\mu}^{a}(p, p)=-\frac{1}{2} \partial_{\mu} G^{-1}(p) I \otimes \sigma_{a},
$$

or explicitly,

$$
\begin{aligned}
\Gamma_{0}^{a}(p, p) & =\frac{1}{2} I \otimes \sigma_{a}, \\
\Gamma_{i}^{a} & =-\frac{1}{2} \partial_{i} G^{-1}(p) I \otimes \sigma_{a},
\end{aligned}
$$

where $\partial_{i}$ and $\partial_{\mu}$ stand for $\partial / \partial p_{i}, \partial / \partial p_{\mu}$ from here on, and the extra minus in the first relation is consistent because implicitly $q_{\mu} \Gamma_{\mu}=q_{0} \Gamma_{0}-q_{i} \Gamma_{i}$.

We now calculate $\Pi$ at small $q$. To zeroth order, use of the Ward identity shows that the $J$ - $J$ function gives zero, except when $\mu=\nu=i$. In that case, it reduces to a constant that cancels the diamagnetic term also present in just that case. Hence we require only the part firstorder in $q$. In the expression for $\Pi$ above, we first shift $p \rightarrow p-\frac{1}{2} q$, so that $q$ no longer appears in any bare vertices, but does appear in the Green's functions on both sides of the ladder, between the rungs which are the interaction lines. Hence to first order, we obtain a factor $\pm \frac{1}{2} \partial_{\mu} G=\mp \frac{1}{2} G \partial G^{-1} G$ in place of $G$ in one position in the ladder. Since there may be any number of rungs (including zero) between this and either of the vertices at the ends, the terms can be summed up into a ladder dressing each vertex, evaluated at $q=0$. Hence we obtain to first order

$$
\begin{aligned}
\Pi_{\mu \nu}^{a b}(q)= & -\frac{i}{2} \int \frac{d^{3} p}{(2 \pi)^{3}} \operatorname{tr}\left[\Gamma_{\mu}^{a}(p, p) q_{\lambda} \partial_{\lambda} G \Gamma_{\nu}^{b}(p, p) G(p)\right. \\
& \left.-\Gamma_{\mu}^{a}(p, p) G(p) \Gamma_{\nu}^{b}(p, p) q_{\lambda} \partial_{\lambda} G\right] .
\end{aligned}
$$

Using the Ward identity, this becomes

$$
\begin{aligned}
\Pi_{\mu \nu}^{a b}(q)= & \frac{i}{8} q_{\lambda} \int \frac{d^{3} p}{(2 \pi)^{3}} \operatorname{tr}\left\{\left(I \otimes \sigma_{a}\right)\left(I \otimes \sigma_{b}\right) G \partial_{\mu} G^{-1}\right. \\
& \left.\times\left[G \partial_{\lambda} G^{-1}, G \partial_{\nu} G^{-1}\right]\right\}
\end{aligned}
$$

Since the $G$ 's are independent of the spin-space indices, the explicit $\sigma$ 's factor off, and the result is $\delta^{a b}$ times a spin-independent part. The latter can be simplified using the BCS-Hartree-Fock form of $G$, by writing the latter as

$$
G(p)=\frac{p_{0}+\mathbf{E}_{\mathbf{p}} \cdot \boldsymbol{\sigma} \otimes I}{p_{0}^{2}-E_{\mathbf{p}}^{2}} .
$$

The spin-independent factor contains $\epsilon_{\mu \nu \lambda}$ since it is antisymmetric in these labels. Keeping track of the signs, we find for the quadratic term in the induced action

$$
\frac{1}{4 \pi} \frac{\mathcal{M}}{4} \int d^{3} r A_{\mu}^{a} \frac{\partial A_{\nu}^{a}}{\partial r_{\lambda}} \epsilon_{\mu \nu \lambda},
$$

with $\mathcal{M}$ given by the topological invariant

$$
\mathcal{M}=\int \frac{d^{2} p}{8 \pi} \epsilon_{i j} \mathbf{E}_{\mathbf{p}} \cdot\left(\partial_{i} \mathbf{E}_{\mathbf{p}} \times \partial_{j} \mathbf{E}_{\mathbf{p}}\right) / E_{\mathbf{p}}^{3} .
$$

The right hand side is exactly the winding number $m$ discussed in Sec. III, and is an integer as long as $\mathbf{E}$ is a continuous, differentiable function of $\mathbf{p}$; it is 2 for the d-wave case.

To ensure $\mathrm{SU}(2)$ gauge invariance, the CS term should include also a term cubic in $A$, with no derivatives. For this term we evaluate the triangle one-loop diagrams with three insertions of $J$, with each vertex dressed by the ladder series. Setting the external momenta to zero, the Ward identity can be used for all three vertices, and the result can be seen to be

$$
\begin{aligned}
\Pi_{\mu \nu \lambda}^{a b c}(0,0)= & -\frac{1}{24} \int \frac{d^{3} p}{(2 \pi)^{3}} \operatorname{tr}\left[\left(I \otimes \sigma_{a}\right) G \partial_{\mu} G^{-1}\right. \\
& \left.\times\left\{\left(I \otimes \sigma_{b}\right) G \partial_{\nu} G^{-1},\left(I \otimes \sigma_{c}\right) G \partial_{\lambda} G^{-1}\right\}\right] .
\end{aligned}
$$

The anticommutator $\{$,$\} arises since the result must be$ symmetric under permutations of the index pairs $\mu, a$, etc. The product $\sigma_{a} \sigma_{b} \sigma_{c}$, when traced over the spinspace indices, yields a factor $2 i \epsilon_{a b c}$, which is antisymmetric, and so the remainder must contain $\epsilon_{\mu \nu \lambda}$ to maintain symmetry; the rest of the structure is the same as before. Hence the full result is the $\mathrm{SU}(2) \mathrm{CS}$ term, which 
we write in terms of the $2 \times 2$ matrix vector potentials $A_{\mu}=\frac{1}{2} \sigma_{a} A_{\mu}^{a}$,

$$
\frac{k}{4 \pi} \int d^{3} x \epsilon_{\mu \nu \lambda} \operatorname{tr}\left(A_{\mu} \partial_{\nu} A_{\lambda}+\frac{2}{3} A_{\mu} A_{\nu} A_{\lambda}\right) .
$$

Here $k$ is the conventional notation for the coefficient of such a term, in this same normalization; if we wished to quantize the theory by functionally integrating over $A$, we would need $k=$ an integer. In our case $k=\mathcal{M} / 2=1$ for d-wave.

For the spin-triplet case with an unbroken $\mathrm{U}(1)$ symmetry, we must use the fact that $\Delta_{-\mathbf{k}}=-\Delta_{\mathbf{k}}$. For example, in the two-dimensional A-phase, as occurs in the 331 state in the double-layer FQHE system with zero tunneling, discussed in Sec. III, the pairs are in the isospin $S_{z}=0$ triplet state $\uparrow_{i} \downarrow_{j}+\downarrow_{i} \uparrow_{j}$, and the U(1) symmetry generated by $S_{z}$ is unbroken; we recall that the underlying Hamiltonian is not assumed to have a full SU(2) symmetry. The effective quasiparticle Hamiltonian (38) becomes, in the Nambu-style notation,

$$
\begin{aligned}
K_{\mathrm{eff}}= & \sum_{\mathbf{k}} \Psi_{\mathbf{k}}^{\dagger}\left[\xi_{\mathbf{k}}\left(\sigma_{z} \otimes I\right)+\operatorname{Re} \Delta_{\mathbf{k}}\left(\sigma_{x} \otimes \sigma_{z}\right)\right. \\
& \left.-\operatorname{Im} \Delta_{\mathbf{k}}\left(\sigma_{y} \otimes \sigma_{z}\right)\right] \Psi_{\mathbf{k}} .
\end{aligned}
$$

The U(1) vector potential $A_{\mu}$ couples to $S_{z}$, and the vertex functions contain $I \otimes \sigma_{z}$, which commutes with the BCS-Hartree-Fock Green's function $G$. The tensors appearing in the three terms in $K_{\text {eff }}$ obey the same algebra as the three in that for the spin-singlet case (where they were trivial in the second factor), and as in that case commute with $I \otimes \sigma_{z}$. Consequently, the derivation for the induced action to quadratic order in $A_{\mu}$ is similar to that for the $\mathrm{SU}(2)$ singlet case above, and the traces in the Nambu indices can be carried out with the same result as before, to obtain the abelian CS term

$$
\frac{1}{4 \pi} \mathcal{M} \int d^{3} r A_{\mu} \frac{\partial A_{\nu}}{\partial r_{\lambda}} \epsilon_{\mu \nu \lambda}
$$

and no cubic term. In this case, $\mathcal{M}$ is again given by the winding number $m$ which is 0 or \pm 1 in the p-wave strong and weak-pairing phases (respectively) discussed in this paper.

We note that the effect of the vertex corrections we included as ladder series is to renormalize the $q=0$ vertices as shown in eq. (A23) for the spin-singlet case, and use these in one-loop diagrams with no further corrections. This corresponds to the minimal coupling $p \rightarrow p-A$ in the action, as one would expect by gauge invariance. If we assume such a coupling, and treat the low-energy, long-wavelength theory near the weak-strong transition as Dirac fermions with relativistic dispersion and minimal coupling to the external gauge field, then the expression for $\mathcal{M}$ as an integral over $\mathbf{p}$ covers only half the sphere in $\mathbf{n}$ space, and we would get \pm 1 (d-wave), $\pm 1 / 2$ (p-wave). The missing part results from the ultraviolet regulator in the field theory version of the calculation 82], or from a second fermion with a fixed mass in some lattice models [100]. In our calculation, the remainder is provided by the ultraviolet region, where $\Delta_{\mathbf{k}} \rightarrow 0$ as $\mathbf{k} \rightarrow \infty$. At the transition, $\mu=0$, the map is discontinuous and covers exactly half the sphere in the p-wave case, so $\mathcal{M}=1 / 2$, as in other problems. In the $\mathrm{d}$-wave case with rotational symmetry, the value of $\left|v_{\mathbf{k}} / u_{\mathbf{k}}\right|$ as $\mathbf{k} \rightarrow 0$ is nonuniversal, as noted in Sec. IVA, and hence so is the value of $\sigma_{x y}^{s}$ at the transition. This is a consequence of the non-relativistic form of the dispersion relation of the low-energy fermions in this case. We may also note that for a paired system on a lattice, as in models of high $T_{c}$ superconductors, a similar calculation will give an integral over the Brillouin zone, which is a torus $T^{2}$, instead of the $\mathbf{k}$ plane which can be compactified to $S^{2}$. But maps from $T^{2}$ to $S^{2}$ are again classified by the integers, and the integer winding number is given by the same expression, so quantization is unaffected.

We can also argue that the quantization result away from a transition is exact in a translationally-invariant system, at least in all orders in perturbation theory. For this we use the form in eq. A26 or (A30), where the Ward identity for the vertex has been used. Diagrammatically, it is clear that the exact expression can be similarly written, using the exact (i.e., all orders in perturbation) Green's function and vertex function. (This is also true when the CS gauge field interaction is included.) The Ward identity that relates them is exact, and the result for $\sigma_{x y}^{s}$ is of the same form as shown. The next step, the frequency integrals, cannot be done explicitly in this case, because the precise form of the Green's function is unknown, and the analogs of $\xi_{\mathbf{k}}, \Delta_{\mathbf{k}}$ (or of $u_{\mathbf{k}}, v_{\mathbf{k}}$ ) do not exist. The latter do not exist because in general the poles in the Green's function, which would represent the quasiparticles, are broadened by scattering processes, except for the lowest energies for kinematical reasons. However, the form in eq. (A26) is itself a topological invariant, as we will now argue. As long as there is a gap in the support of the spectral function of $G, G(p)$ is continuous and differentiable on the imaginary frequency axis, and tends to $I \otimes I / p_{0}$ as $p_{0} \rightarrow \pm i \infty$. Thus $G^{-1}$ exists and never vanishes. Considering the spin-singlet case for convenience, the spin-space structure is trivial, so we may perform the corresponding traces, and then $G$ or $G^{-1}$ is a $2 \times 2$ matrix, with the same reality properties on the imaginary $p_{0}$ axis as in the BCS-Hartree-Fock approximation. (The spin-triplet case should work out similarly, because of the algebraic structure already mentioned.) It thus represents a real non-zero 4-component vector, in $\mathbf{R}^{4}-0$, which topologically is the same as $S^{3}$. $S^{3}$ is obtained by dividing $G$ by its norm, $\left(\operatorname{tr} G^{\dagger} G\right)^{1 / 2}$, and the normalized $G$ is a $2 \times 2$ unitary matrix with determinant -1 , so it lies in $S^{3}$. The $\mathbf{k}$ space can be compactified to $S^{2}$ as before, and the frequency variable can be viewed as an 
element of the interval $\mathcal{I}=(-1,1)$, so the integral is over $S^{2} \times \mathcal{I}$. However, since the limit of the Green's function as $p_{0} \rightarrow \pm i \infty$ for fixed $\mathbf{k}$ is independent of $\mathbf{k}$, we can view this as simply $S^{3}$. Thus we are dealing with maps from $S^{3}$ to $S^{3}$, the equivalence classes of which are classified by the homotopy group $\pi_{3}\left(S^{3}\right)=\mathbf{Z}$. The integral we have obtained simply calculates the integer winding number or Pontryagin index of the map, when properly normalized ( $G$ can be normalized to lie in $\mathrm{SU}(2)$ without affecting the integral). This establishes the quantization of $\sigma_{x y}^{s}$ in a translationally-invariant system with a gap, at least to all orders in perturbation theory, and probably can be made fully non-perturbative (as the Ward identity is already).

[1] J. Bardeen, L.N. Cooper, and J.R. Schrieffer, Phys. Rev. 106, 162 (1957); 108, 1175 (1957).

[2] J.R. Schrieffer, Theory of Superconductivity, (AddisonWesley, Reading MA, 1964).

[3] For a review, see e.g., D. Vollhardt and P. Wölfle, The Superfluid Phases of Helium 3, (Taylor and Francis, London, 1990).

[4] G.E. Volovik, Zh. Eksp. Teor. Fiz. 94, 123 (1988) [Sov. Phys. JETP 67, 1804 (1988)].

[5] G.E. Volovik and V.M. Yakovenko, J. Phys. Cond. Matter 1, 5263 (1989).

[6] G.E. Volovik, Physica B 162, 222 (1990).

[7] G.E. Volovik, Sov. Phys. JETP Lett. 51, 125 (1990).

[8] G.E. Volovik, Sov. Phys. JETP Lett. 55, 368 (1992).

[9] For a review, see, e.g., The Quantum Hall Effect, edited by R.E. Prange and S.M. Girvin (Second Edition, Springer-Verlag, New York, 1990).

[10] R.B. Laughlin, Phys. Rev. Lett. 50, 1395 (1983).

[11] B.I. Halperin, Helv. Phys. Acta, 56, 75 (1983).

[12] R. Morf, N. d'Ambrunil, and B.I. Halperin, Phys. Rev. B 34, 3037 (1986).

[13] F.D.M. Haldane and E.H. Rezayi, Phys. Rev. Lett. 60, 956, 1886 (E) (1988).

[14] G. Moore and N. Read, Nucl. Phys. B360, 362 (1991); N. Read and G. Moore, Prog. Theor. Phys. (Kyoto) Supp. 107, 157 (1992).

[15] F.D.M. Haldane, Phys. Rev. Lett. 51, 605 (1983), and in Ref. [9].

[16] S.M. Girvin in Ref. [9].

[17] S.M. Girvin and A.H. MacDonald, Phys. Rev. Lett. 58, 1252 (1987).

[18] N. Read, Bull. Am. Phys. Soc, 32, 923 (1987).

[19] R.B. Laughlin, Phys. Rev. Lett. 60, 2677 (1988).

[20] S.C. Zhang, T.H. Hansson, and S. Kivelson, Phys. Rev. Lett. 62, 82 (1989).

[21] N. Read, Phys. Rev. Lett. 62, 86 (1989).

[22] J.K. Jain, Phys. Rev. Lett. 63, 199 (1989); Phys. Rev. B 40, 8079 (1989); ibid. 41, 7653 (1990).

[23] D.-H. Lee and M.P.A. Fisher, Phys. Rev. Lett. 63, 903
(1989).

[24] A. Lopez and E. Fradkin, Phys. Rev. B 44, 5246 (1991).

[25] B.I. Halperin, P.A. Lee, and N. Read, Phys. Rev. B 47, 7312 (1993).

[26] N. Read, Semicond. Sci. Technol. 9, 1859 (1994) condmat/9501090.

[27] R. Shankar and G. Murthy, Phys. Rev. Lett. 79, 4437 (1997); cond-mat/9802244.

[28] D.-H. Lee, Phys. Rev. Lett. 80, 4745 (1998).

[29] V. Pasquier and F.D.M. Haldane, Nucl. Phys. B 516, 719 (1998).

[30] N. Read, Phys. Rev. B 59, 8084 (1998).

[31] R.L. Willett, J.P. Eisenstein, H.L. Stormer, D.C. Tsui, A.C. Gossard, and J.H. English, Phys. Rev. Lett. 59, 1776 (1987).

[32] M. Greiter, X.-G. Wen and F. Wilczek, Phys. Rev. Lett. 66, 3205 (1991); Nucl. Phys. B374, 567 (1992).

[33] R. Morf, Phys. Rev. Lett. 80, 1505 (1998).

[34] E.H. Rezayi and F.D.M. Haldane, cond-mat/9906137.

[35] M. Greiter, X.-G. Wen, and F. Wilczek, Phys. Rev. B 46, 9586 (1992).

[36] B.I. Halperin, Surface Sci. 305, 1 (1994).

[37] Y.W. Suen, L.W. Engel, M.B. Santos, M. Shayegan, and D.C. Tsui, Phys. Rev. Lett. 68, 1379 (1992); J.P. Eisenstein, G.S. Boebinger, L.N. Pfeiffer, K.W. West, and Song He, Phys. Rev. Lett. 68, 1383 (1992).

[38] S. He, X.-C. Xie, S. Das Sarma, and F.-C. Zhang, Phys. Rev. B 43, 9339 (1991); S. He, S. Das Sarma, and X.C. Xie, ibid. 47, 4394 (1993).

[39] E. Witten, Commun. Math. Phys. 121, 351 (1989).

[40] X.-G. Wen, Phys. Rev. Lett. 70, 355 (1993).

[41] X.-G. Wen and Y.-S. Wu, Nucl. Phys. B419 [FS], 455 (1994); X.-G. Wen, Y.-S. Wu and Y. Hatsugai, Nucl. Phys. B422 [FS], 476 (1994).

[42] M. Milovanović and N. Read, Phys. Rev. B 53, 13559 (1996).

[43] C. Nayak and F. Wilczek, Nucl. Phys. B479, 529 (1996).

[44] N. Read and E. Rezayi, Phys. Rev. B 54, 16864 (1996).

[45] V. Gurarie, M. Flohr and C. Nayak, Nucl. Phys. B 498, 513 (1998).

[46] V. Gurarie and C. Nayak, Nucl. Phys. B (1998); condmat/9706227.

[47] E. Fradkin, C. Nayak, A. Tsvelik, and F. Wilczek, Nucl. Phys. B 516, 704 (1998); E. Fradkin, C. Nayak, and K. Schoutens, Nucl. Phys. B 546, 711 (1999); D.C. Cabra, E. Fradkin, G.L. Rossini, and F.A. Schaposnik, condmat/9905192; X.-G. Wen, cond-mat/9811111.

[48] N. Read, Phys. Rev. Lett. 65, 1502 (1990).

[49] B. Blok and X.-G. Wen, Phys. Rev. B 42, 8133, 8145 (1990); ibid. 43, 8337 (1991).

[50] P.G. de Gennes, Superconductivity of Metals and Alloys, (Addison-Wesley, Reading, MA, 1989).

[51] R. Oppermann, Physica A 167, 301 (1990).

[52] A. Altland and M. R. Zirnbauer, Phys. Rev. B 55, 1142 (1997); M. R. Zirnbauer, J. Math. Phys. 37, 4986 (1996).

[53] T. Senthil, M.P.A. Fisher, L. Balents, and C. Nayak, Phys. Rev. Lett. 81, 4704 (1998).

[54] R. Bundschuh, C. Cassanello, D. Serban, and M.R. Zirnbauer, Phys. Rev. B 59, 4382 (1999).

[55] T. Senthil and M.P.A. Fisher, cond-mat/9810238. 
[56] V. Kagalovsky, B. Horovitz, Y. Avishai, and J.T. Chalker, Phys. Rev. Lett. 82, 3516 (1999).

[57] T. Senthil, J.B. Marston, and M.P.A. Fisher, condmat/9902062.

[58] I.A. Gruzberg, A.W.W. Ludwig, and N. Read, Phys. Rev. Lett. 82, 4524 (1999).

[59] R.B. Laughlin, Phys. Rev. Lett. 80, 5188 (1998).

[60] J. Goryo and K. Ishikawa, cond-mat/9812412.

[61] N. Read and D. Green, Bull. Am. Phys. Soc. 44, 304 (1999).

[62] Some of our results have also been found independently by T. Senthil and M.P.A. Fisher (unpublished), and by X.-G. Wen (unpublished).

[63] P.W. Anderson, Phys. Rev. 110, 827 (1958); ibid., 112, 1900 (1958).

[64] F.D.M. Haldane, Phys. Rev. Lett. 55, 2095 (1985).

[65] We are grateful to T.-L. Ho for suggesting that we consider a domain wall as a model for the edge.

[66] R. Jackiw and C. Rebbi, Phys. Rev. D 13, 3398 (1976).

[67] C. Caroli, P.G. de Gennes, and J. Matricon, Phys. Lett. 9, 307 (1964); J. Bardeen, R. Kümmel, A.E. Jacobs, and L. Tewordt, Phys. Rev. 187, 556 (1969); P.G. de Gennes, Sec. 5.2 in Ref. 50.

[68] N.B. Kopnin and M.M. Salomaa, Phys. Rev. B 44, 9667 (1991).

[69] See a general relativity text, or, e.g., M.B. Green, J.H. Schwartz, and E. Witten, Superstring Theory, Vol. 2: Loop Amplitudes, Anomalies, and Phenomenology, (Cambridge University Press, Cambridge, England, 1987), Sec. 12.1.

[70] X.-G. Wen and Q. Niu, Phys. Rev. B 41, 9377 (1990).

[71] T.-L. Ho, Phys. Rev. Lett. 75, 1186 (1995).

[72] L. Belkhir, X.-G. Wu, and J.K. Jain, Phys. Rev. B 48, 15245 (1993).

[73] M. Milovanović and N. Read, Phys. Rev. B 56, 1461 (1997).

[74] D.-H. Lee and C.L. Kane, Phys. Rev. Lett. 64, 1313 (1990).

[75] F.D.M. Haldane and E.H. Rezayi, unpublished.

[76] See Appendix B of Ref. 44.

[77] D. Green, N. Read and E. Rezayi, (unpublished).

[78] X.-G. Wen, Int. J. Mod. Phys. B6, 1711 (1992).

[79] F.D.M. Haldane and D.P. Arovas, Phys. Rev. B 52, 4223 (1995); K. Yang, L.K. Warman, and S.M. Girvin, Phys. Rev. Lett. 70, 2641 (1993).

[80] D.J. Thouless, M. Kohmoto, M.P. Nightingale, and M. Den Nijs, Phys. Rev. Lett. 49, 405 (1982).

[81] J. Bellissard, A. van Elst, and H. Schulz-Baldes, J. Math. Phys. 35, 5373 (1994).

[82] A.N. Redlich, Phys. Rev. Lett. 52, 18 (1984); I. Affleck, J. Harvey, and E. Witten, Nucl. Phys. B 206, 413 (1982).

[83] B.I. Halperin, Phys. Rev. B 25, 2185 (1982).

[84] F.D.M. Haldane, Phys. Rev. Lett. 74, 2090 (1995).

[85] S. Xiong, N. Read, and A.D. Stone, Phys. Rev. B 56, 3982 (1997).

[86] C. Callan and J. Harvey, Nucl. Phys. B 250, 427 (1985).

[87] G.E. Volovik, Pis'ma ZETF 66, 492 (1997); condmat/9709084.

[88] E. Witten, Commun. Math. Phys. 92, 455 (1984).

[89] C.L. Kane and M.P.A. Fisher, Phys. Rev. B 55, 15832
(1997).

[90] H.W.J. Blöte, J.L. Cardy, and M.P. Nightingale, Phys. Rev. Lett. 56, 742 (1986); I. Affleck, ibid., 56, 746 (1986).

[91] L.G.C. Rego and G. Kirczenow, Bull. Am. Phys. Soc. 44, 212 (1999).

[92] A. Belavin, A. Polyakov, and A. Zamolodchikov, Nucl. Phys. B241, 33 (1984).

[93] L. Alvarez-Gaumé and E. Witten, Nucl. Phys. B 234, 269 (1983); L. Alvarez-Gaumé, S. Della Pietra, and G. Moore, Ann. Phys. (NY) 163, 288 (1985).

[94] H. Verlinde, Nucl. Phys. B 337, 652 (1990).

[95] F.D.M. Haldane and Y.-S. Wu, Phys. Rev. Lett. 55, 2887 (1985).

[96] See A.M.M. Pruisken, in [9], and references therein.

[97] T. Senthil and M.P.A. Fisher, cond-mat/9906290.

[98] I.A. Gruzberg, A.W.W. Ludwig, and N. Read, unpublished.

[99] S. Helgason, Differential Geometry, Lie Groups, and Symmetric Spaces, (Academic Press, New York, NY, 1978), Sections IV.4, V.2.

[100] A.W.W. Ludwig, M.P.A. Fisher, R. Shankar, and G. Grinstein, Phys. Rev. B 50, 7526 (1994).

[101] Vl.S Dotsenko and V.S. Dotsenko, Adv. Phys. 32, 129 (1983); R. Shankar, Phys. Rev. Lett. 58, 2466 (1987); A.W.W. Ludwig, Phys. Rev. Lett. 61, 2388 (1988).

[102] L. Kadanoff and H. Ceva, Phys. Rev. B 3, 3918 (1971).

[103] A.V. Shytov, L.S. Levitov, and B.I. Halperin, Phys. Rev. Lett. 80, 141 (1998). 